\title{
SELF-AFFINE MULTIFRACTAL SIERPINSKI SPONGES IN $\mathbb{R}^{d}$
}

\author{
L. OLSEN
}

We study self-affine multifractals in $\mathbb{R}^{d}$ using the formalism introduced in [Olsen, A multifractal formalism, Advances in Mathematics, 116 (1996), 82-196]. We prove that new multifractal phenomena, not exhibited by self-similar multifractals in $\mathbb{R}^{d}$, appear in the self-affine case.

\section{Introduction.}

We analyze the multifractal structure of self-affine invariant measures in $\mathbb{R}^{d}$ supported by a particular type of self-affine sets usually called Sierpinski Sponges. Our analysis is based on the multifractal formalism introduced by Olsen in [Ol1]. For a metric space $X$ we denote the family of Borel probability measures on $X$ by $\mathcal{P}(X)$. For $\mu \in \mathcal{P}(X)$ and $x \in X$ we define the upper and lower local dimension of $\mu$ at $x$ by

$$
\bar{\alpha}_{\mu}(x)=\limsup _{r \searrow 0} \frac{\log \mu B(x, r)}{\log r}
$$

resp.

$$
\underline{\alpha}_{\mu}(x)=\liminf _{r \searrow 0} \frac{\log \mu B(x, r)}{\log r}
$$

where $B(x, r)$ denotes the closed ball with centre $x$ and radius $r$. If $\bar{\alpha}_{\mu}(x)$ and $\underline{\alpha}_{\mu}(x)$ agree we refer to the common value as the local dimension of $\mu$ at $x$ and denote it by $\alpha_{\mu}(x)$. For each $\alpha \geq 0$ define $\Delta_{\mu}(\alpha)$ by

$$
\Delta_{\mu}(\alpha)=\left\{x \in \operatorname{supp} \mu \mid \alpha_{\mu}(x)=\alpha\right\}
$$

where $\operatorname{supp} \mu$ denotes the topological support of $\mu$. The main problem in multifractal analysis is to estimate the size of the sets $\Delta_{\mu}(\alpha)$; this is done by introducing the functions $f_{\mu}, F_{\mu}: \mathbb{R}_{+} \rightarrow \mathbb{R}_{+}$defined by

$$
\begin{aligned}
f_{\mu}(\alpha) & =\operatorname{dim} \Delta_{\mu}(\alpha) \\
F_{\mu}(\alpha) & =\operatorname{Dim} \Delta_{\mu}(\alpha)
\end{aligned}
$$


where dim and Dim denote Hausdorff dimension and packing dimension respectively. These and similar functions are generically known as "the multifractal spectrum of $\mu$ ", "the singularity spectrum of $\mu$ ", "the spectrum of scaling indices" or simply "the $f(\alpha)$-spectrum". The function $f(\alpha)=f_{\mu}(\alpha)$ was first explicitly defined by the physicists Halsey et al. in 1986 in their seminal paper [HJKPS]. The reader is referred to Cawley \& Mauldin [CM] or Olsen [O11] for a more detailed historical account of multifractality, and an extensive list of references.

Many recent papers have studied the multifractal structure of self-similar measures. Cawley \& Mauldin $[\mathbf{C M}]$ analyzed the multifractal structure of (non-random) self-similar measures, and Edgar \& Mauldin [EM] and Olsen [Ol1] investigated the multifractal structure of (non-random) graph directed self-similar measures. Riedi [Re2] has studied self-similar multifractals generated by a countable infinite number of similarities. A recent research monograph by Olsen [O12] presents a detailed multifractal analysis of random graph directed self-similar measures based on the formalism introduced in [O11]. Independently Falconer [Fa4] and later Arbeiter \& Patzschke [PA] have studied random self-similar multifractals.
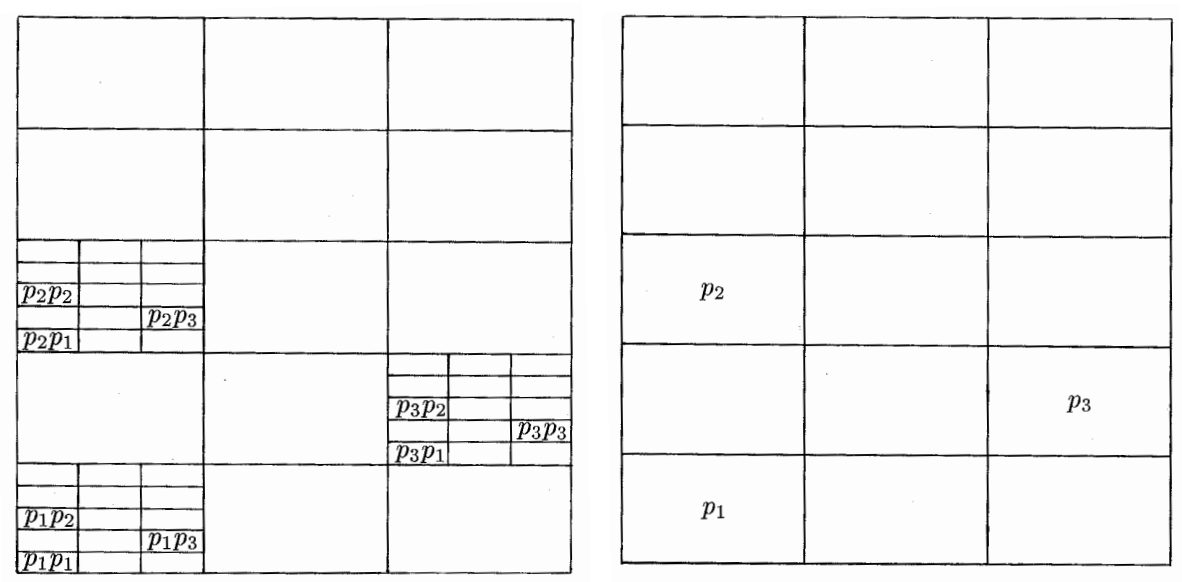

Figure 1.1. The first two stages in the construction of a self-affine measure. In this example $d=2, n_{1}=2, n_{2}=5, \mathbf{B}$ consists of 3 boxes and

$$
\mathbf{p}=\left(p_{1}, p_{2}, p_{3}\right) \text {. }
$$


We note that all the papers [AP, CM, EM, Fa4, Ol1, Ol2, Re2] analyze the multifractal structure of self-similar (or graph directed self-similar) measures. In this paper we focus on the multifractal structure of self-affine measures $\mu$ in $\mathbb{R}^{d}$ supported by a particular type of self-affine sets usually called Sierpinski Sponges - thus we will call the measures $\mu$ that we study for self-affine multifractal Sierpinski Sponges. Let $1<n_{1} \leq n_{2} \leq \cdots \leq n_{d}$ be integers. By considering $n_{l}-1(d-1)$-dimensional hyperplanes parallel to the hyperplane spanned by $\left(\left(\delta_{i 1}\right)_{i=1, \ldots, d}, \ldots,\left(\delta_{i, l-1}\right)_{i=1, \ldots, d},\left(\delta_{i, l+1}\right)_{i=1, \ldots, d}, \ldots\right.$, $\left(\delta_{i d}\right)_{i=1, \ldots, d}$ ) (here $\delta_{i j}$ denotes the Kronecker delta) for each $l=1, \ldots, d$, we partition the unit cube $[0,1]^{d}$ into $n_{1} \cdots n_{d}$ congruent boxes with sidelengths $\frac{1}{n_{1}}, \ldots, \frac{1}{n_{d}}$. Let $\mathbf{B}$ be a subcollection of these boxes and let $\mathbf{p}=\left(p_{B}\right)_{B \in \mathbf{B}}$ be a probability vector indexed by $\mathbf{B}$. Erase all the boxes not contained in $\mathbf{B}$, and divide a unit mass between the remaining boxes in the ratio determined by the probability vector $\mathbf{p}$. Next partition each of the remaining boxes $B$ into $n_{1} \cdots n_{d}$ congruent subboxes of $B$ with sidelengths $\frac{1}{n_{1}^{2}}, \ldots, \frac{1}{n_{d}^{2}}$, again keeping only those which corresponds to $\mathbf{B}$, and divide the mass of $B$ between the remaining subboxes of $B$ in the ratio determined by the probability vector p. Continuing this process infinitely, a compact set $K$ and a probability measure $\mu$ supported on $K$ are obtained, see Figure 1.1. The set $K$ is called a self-affine Sierpinski Sponge, and the measure $\mu$ is called a self-affine multifractal Sierpinski Sponge. In this paper we study the multifractal structure of the measure $\mu$. Our analysis will be based on the multifractal formalism introduced by Olsen [O11]. In particular we find, assuming separation condition (II) (introduced in Section 4),

1) the Hausdorff spectrum $f_{\mu}$ of $\mu$;

2) the multifractal box dimensions of $\mu$;

3) the generalized Renyi dimensions of $\mu$;

4) the multifractal dimension functions $b_{\mu}, B_{\mu}$ and $\Lambda_{\mu}$ introduced in [Ol1];

5) a sufficient condition guaranteeing that the multifractal Hausdorff and multifractal packing measures, $\mathcal{H}_{\mu}^{q, b_{\mu}(q)}(\operatorname{supp} \mu)$ and $\mathcal{P}_{\mu}^{q, B_{\mu}(q)}(\operatorname{supp} \mu)$ introduced in [O11], are positive and finite.

In the 2-dimensional case, separation condition (II) states that if a column of rectangles contains a box from $\mathbf{B}$, then the two immediately adjacent columns of rectangles do not contain any boxes from B, c.f. Figure 1.1. This separation condition is unfortunately very strong and it would be very desirable if it could be weakened or omitted.

We prove that self-affine multifractal Sierpinski Sponges possess some wellknown multifractal characteristica; in particular we prove the following:

1) There exist two numbers $0 \leq \underline{a} \leq \bar{a}$ such that $\Delta_{\mu}(\alpha)=\varnothing$ for $\alpha \notin[\underline{a}, \bar{a}]$, and $f_{\mu}(\alpha)>0$ for $\left.\alpha \in\right] \underline{a}, \bar{a}[$. 
2) the multifractal spectrum function $f_{\mu}$ equals the Legendre transform $\beta^{*}$ of a certain auxiliary function $\beta$ defined explicitely in terms of the numbers $n_{1}, \ldots, n_{d}$ and the probability vector $\mathbf{p}$. In particular, $f_{\mu}$ is concave on its support.

However, we also prove that new multifractal phenomena, not exhibited by self-similar multifractals in $\mathbb{R}^{d}$, appear in the self-affine case; in particular we prove the following:

1) The Hausdorff multifractal dimension function $b_{\mu}$ and the packing multifractal dimension function $B_{\mu}$ do not necessarily coincide; in fact, for a fixed $q \in \mathbb{R}, b_{\mu}(q)$ and $B_{\mu}(q)$ coincide if and only if condition $\left(\mathrm{I}_{q}\right)$ introduced in Section 4 is satisfied. This phenomenon is in sharp contrast to the self-similar case in which $b_{\mu}=B_{\mu}$ by [Ol1, Theorem 5.1].

2) We conjecture that the multifractal Hausdorff measure $\mathcal{H}_{\mu}^{q, b_{\mu}(q)}(\operatorname{supp} \mu)$ and the multifractal packing measure $\mathcal{P}_{\mu}^{q, B_{\mu}(q)}(\operatorname{supp} \mu)$ are not necessarily positive and finite. For a fixed $q \in \mathbb{R}$, condition $\left(\mathrm{I}_{q}\right)$ implies that $0<\mathcal{H}_{\mu}^{q, b_{\mu}(q)}(\operatorname{supp} \mu)<\infty$ and $0<\mathcal{P}_{\mu}^{q, B_{\mu}(q)}(\operatorname{supp} \mu)<\infty$, and we conjecture that if $\left(\mathrm{I}_{q}\right)$ is not satisfied, then $\mathcal{H}_{\mu}^{q, b_{\mu}(q)}(\operatorname{supp} \mu)=$ $\mathcal{P}_{\mu}^{q, B_{\mu}(q)}(\operatorname{supp} \mu)=\infty$; cf. Conjecture 4.1.10 and Conjecture 4.1.11. This phenomenon is in sharp contrast to the self-similar case in which $\mathcal{H}_{\mu}^{q, b_{\mu}(q)}(\operatorname{supp} \mu)$ and $\mathcal{P}_{\mu}^{q, B_{\mu}(q)}(\operatorname{supp} \mu)$ are positive and finite for all $q \in$ $\mathbb{R}$ by [011, Theorem 5.1].

3) The Legendre transform $B_{\mu}^{*}$ of $B_{\mu}$ does not necessarily attain the constant value $-\infty$ outside the set $[\underline{a}, \bar{a}]$. There exist self-affine measures $\mu$ such that $0<B_{\mu}^{*}(\alpha)$ for all $\alpha \in[\underline{A}, \underline{a}] \cup[\bar{a}, \bar{A}]$ where at least one of the intervals $[\underline{A}, \underline{a}]$ or $[\bar{a}, \bar{A}]$ is non-degenerate. This phenomenon is in sharp contrast to the self-similar case in which $B_{\mu}^{*}(\alpha)=-\infty$ for all $\alpha \notin[\underline{a}, \bar{a}]$ by [O11, Theorem 5.1]. (We remark that a very similar situation arises in multifractal analysis of random (graph directed) self-similar measures: generically there exist (cf. [O12]) numbers $0 \leq$ $\underline{a} \leq a_{\min } \leq a_{\max } \leq \bar{a}$ such that for each fixed $\left.\alpha \in\right] \underline{a}, a_{\min }[\cup] a_{\max }, \bar{a}[$, almost all self-similar measures $\nu$ satisfy $-\infty<b_{\nu}^{*}(\alpha)=B_{\nu}^{*}(\alpha)<0=$ $\left.f_{\nu}(\alpha)=F_{\nu}(\alpha).\right)$

We note that our results, due to the use of the generalized multifractal Hausdorff and packing measures introduced in [O11], appear as natural multifractal generalizations of some of the main results on self-affine sets by Bedford $[\mathbf{B e}]$, McMullen [McM], Kenyon \& Peres [KP] and Peres [Pe1, Pe2], in particular Kenyon \& Peres [KP, Theorem 1.2 and Proposition 1.3] and Peres [Pe2, Theorem 1.1.(ii)].

King $[\mathbf{K i}]$ have determined the Hausdorff spectrum $f_{\mu}$ for self-affine multifractal Sierpinski Sponges in $\mathbb{R}^{2}$. In this paper we extend King's results to $\mathbb{R}^{d}$ 
(it should be noted that the extension from $\mathbb{R}^{2}$ to $\mathbb{R}^{d}$ is not merely a technical extension, c.f. also Kenyon \& Peres [KP, remark just above Theorem 1.2]) and, in addition, investigate the multifractal box dimensions of $\mu$, the generalized multifractal Hausdorff and packing measures, $\mathcal{H}_{\mu}^{q, t}$ and $\mathcal{P}_{\mu}^{q, t}$, and the generalized multifractal dimension functions $b_{\mu}$ and $B_{\mu}$ introduced in [O11]. Schmeling \& Siegmund-Schultze [SS] have studied certain self-affine multifractals. However, Schmeling \& Siegmund-Schultze's approach is different from our approach. Schmeling \& Siegmund-Schultze consider a probability vector $\left(p_{i}\right)_{i=1, \ldots, N}$ and a family of affine maps $\left(x \rightarrow A_{i} x+a_{i}\right)_{i=1, \ldots, N}$ where $A_{i}$ are linear contractions of $\mathbb{R}^{d}$ with $\left\|A_{i}\right\|<\frac{1}{3}$ and $a_{i} \in \mathbb{R}^{d}$. They study, for Lebesgue almost all translation vectors $\left(a_{i}\right)_{i=1, \ldots, N} \in\left(\mathbb{R}^{d}\right)^{N}$, a part of the Hausdorff spectrum function $f_{\mu}$ of the self-affine measure $\mu$ generated by the maps $\left(x \rightarrow A_{i} x+a_{i}\right)_{i=1, \ldots, N}$ and the probabilities $\left(p_{i}\right)_{i=1, \ldots, N}$ (i.e. $\mu$ is the unique probability measure on $\mathbb{R}^{d}$ satisfying the self-affine equation $\mu=\sum_{i} p_{i} \mu \circ S_{i}^{-1}$ where $\left.S_{i}(x)=A_{i}(x)+a_{i}\right)$. Schmeling \& SiegmundSchultze's approach can be viewed as an attempt to generalize some of Falconer's [Fa1, Fa3] results on self-affine sets to the multifractal case. Falconer also considers a family of affine maps $\left(x \rightarrow A_{i} x+a_{i}\right)_{i=1, \ldots, N}$ where $A_{i}$ are linear contractions of $\mathbb{R}^{d}$ with $\left\|A_{i}\right\|<\frac{1}{3}$ and $a_{i} \in \mathbb{R}^{d}$. Falconer then shows that, for Lebesgue almost all translation vectors $\left(a_{i}\right)_{i=1, \ldots, N} \in\left(\mathbb{R}^{d}\right)^{N}$, the self-affine set $K$ generated by the maps $\left(x \rightarrow A_{i} x+a_{i}\right)_{i=1, \ldots, N}$ (i.e. $K$ is the unique non-empty compact subset of $\mathbb{R}^{d}$ satisfying the self-affine equation $K=\cup_{i} S_{i}(K)$ where $\left.S_{i}(x)=A_{i}(x)+a_{i}\right)$ has equal Hausdorff dimension and box dimension and presents an asymptotic formula for this dimension. Finally we note that Riedi [Ri1] has computed the multifractal box dimensions of a class of self-affine multifractal.

We will now give a brief description of the organization of the paper. In Section 2 we recall the multifractal formalism introduced in [Ol1], and define the notion of a self-affine set and a self-affine measure. In Section 3 we introduce two auxiliary functions, $\beta$ and $\gamma$, and study their properties. Section 4 contains the statements of our main results formulated in terms of the auxiliary functions $\beta$ and $\gamma$. Section 5 contains an example. In Section 6 we present the proofs of our main results.

\section{The Setting.}

\subsection{The multifractal measures $\mathcal{H}_{\mu}^{q, t}$ and $\mathcal{P}_{\mu}^{q, t}$.}

This section gives a brief summary of the main results in [O11]. We first recall the definition of the Hausdorff measure, the centered Hausdorff measure and the packing measure. Let $X$ be a metric space, $E \subseteq X$ and $\delta>0$. A countable family $\mathcal{B}=\left(B\left(x_{i}, r_{i}\right)\right)_{i}$ of closed balls in $X$ is called a 
centered $\delta$-covering of $E$ if $E \subseteq \cup_{i} B\left(x_{i}, r_{i}\right), x_{i} \in E$ and $0<r_{i}<\delta$ for all $i$. The family $\mathcal{B}$ is called a centered $\delta$-packing of $E$ if $x_{i} \in E, 0<r_{i}<\delta$ and $B\left(x_{i}, r_{i}\right) \cap B\left(x_{j}, r_{j}\right)=\varnothing$ for all $i \neq j$. Let $E \subseteq X, t \geq 0$ and $\delta>0$. Now put

$$
\mathcal{H}_{\delta}^{t}(E)=\inf \left\{\sum_{i} \operatorname{diam}\left(E_{i}\right)^{t} \mid E \subseteq \bigcup_{i=1}^{\infty} E_{i}, \operatorname{diam} E_{i}<\delta\right\} .
$$

The $t$-dimensional Hausdorff measure $\mathcal{H}^{t}(E)$ of $E$ is defined by

$$
\mathcal{H}^{t}(E)=\sup _{\delta>0} \mathcal{H}_{\delta}^{t}(E)
$$

The reader is referred to [Fa2] for more information on $\mathcal{H}^{t}$. We will now define the packing measure. Write

$$
\overline{\mathcal{P}}_{\delta}^{t}(E)=\sup \left\{\sum_{i=1}^{\infty}\left(2 r_{i}\right)^{t} \mid\left(B\left(x_{i}, r_{i}\right)\right)_{i} \text { is a centered } \delta \text {-packing of } E\right\} .
$$

The $t$-dimensional prepacking measure $\overline{\mathcal{P}}^{t}(E)$ of $E$ is defined by

$$
\overline{\mathcal{P}}^{t}(E)=\inf _{\delta>0} \overline{\mathcal{P}}_{\delta}^{t}(E)
$$

The set function $\overline{\mathcal{P}}^{t}$ is not necessarily countable subadditive, and hence not necessarily an outer measure, c.f. [TT] or [Fa2]. But $\overline{\mathcal{P}}^{t}$ give rise to a Borel measure, namely the $t$-dimensional packing measure $\mathcal{P}^{t}(E)$ of $E$, as follows

$$
\mathcal{P}^{t}(E)=\inf _{E \subseteq \cup i=1} \sum_{E_{i}} \sum_{i=1}^{\infty} \overline{\mathcal{P}}^{t}\left(E_{i}\right)
$$

The packing measure was introduced by Taylor and Tricot in [TT] using centered $\delta$-packings of open balls, and by Raymond and Tricot in $[\mathbf{R T}]$ using centered $\delta$-packings of closed balls.

Also recall that the Hausdorff dimension $\operatorname{dim}(E)$, the packing dimension $\operatorname{Dim}(E)$ and the logarithmic index $\Delta(E)$ of $E$ is defined by

$$
\begin{aligned}
\operatorname{dim}(E) & =\sup \left\{t \geq 0 \mid \mathcal{H}^{t}(E)=\infty\right\} \\
\operatorname{Dim}(E) & =\sup \left\{t \geq 0 \mid \mathcal{P}^{t}(E)=\infty\right\} \\
\Delta(E) & =\sup \left\{t \geq 0 \mid \overline{\mathcal{P}}^{t}(E)=\infty\right\} .
\end{aligned}
$$

We refer the reader to $[\mathbf{T r}]$ and $[\mathbf{R T}]$ for more information on the centered Hausdorff measure, the packing measure and the packing dimension. 
Olsen [Ol1] suggested that some multifractal generealizations of the (centered) Hausdorff measure and the packing measure might be useful in multifractal analysis. For $q \in \mathbb{R}$ define $\varphi_{q}:\left[0, \infty\left[\rightarrow \overline{\mathbb{R}}_{+}=[0, \infty]\right.\right.$ by

$$
\begin{aligned}
& \varphi_{q}(x)= \begin{cases}\infty & \text { for } x=0 \\
x^{q} & \text { for } 0<x\end{cases} \\
& \varphi_{q}(x)=\begin{array}{ll}
1 & \text { for } q<0
\end{array} \\
& \varphi_{q}(x)= \begin{cases}0 & \text { for } x=0 \\
x^{q} & \text { for } 0<x\end{cases}
\end{aligned}
$$

For $\mu \in \mathcal{P}(X), E \subseteq X, q, t \in \mathbb{R}$ and $\delta>0$ write

$$
\overline{\mathcal{H}}_{\mu, \delta}^{q, t}(E)=\inf \left\{\sum_{i} \varphi_{q}\left(\mu\left(B\left(x_{i}, r_{i}\right)\right)\right)\left(2 r_{i}\right)^{t} \mid\left(B\left(x_{i}, r_{i}\right)\right)_{i}\right.
$$

is a centered $\delta$-covering of $E\}, E \neq \varnothing$

$$
\begin{aligned}
& \overline{\mathcal{H}}_{\mu, \delta}^{q, t}(\varnothing)=0 \\
& \overline{\mathcal{H}}_{\mu}^{q, t}(E)=\sup _{\delta>0} \overline{\mathcal{H}}_{\mu, \delta}^{q, t}(E) \\
& \mathcal{H}_{\mu}^{q, t}(E)=\sup _{F \subseteq E} \overline{\mathcal{H}}_{\mu}^{q, t}(F) .
\end{aligned}
$$

We also make the dual definitions

$$
\begin{aligned}
& \overline{\mathcal{P}}_{\mu, \delta}^{q, t}(E)=\sup \left\{\sum_{i} \varphi_{q}\left(\mu\left(B\left(x_{i}, r_{i}\right)\right)\right)\left(2 r_{i}\right)^{t} \mid\left(B\left(x_{i}, r_{i}\right)\right)_{i}\right. \\
& \quad \text { is a centered } \delta \text {-packing of } E\}, E \neq \varnothing \\
& \overline{\mathcal{P}}_{\mu, \delta}^{q, t}(\varnothing)=0 \\
& \overline{\mathcal{P}}_{\mu}^{q, t}(E)=\inf _{\delta>0} \overline{\mathcal{P}}_{\mu, \delta}^{q, t}(E) \\
& \mathcal{P}_{\mu}^{q, t}(E)=\inf _{E \subseteq \cup_{i} E_{i}} \sum_{i} \overline{\mathcal{P}}_{\mu}^{q, t}\left(E_{i}\right) .
\end{aligned}
$$

It is proven in [O11] that $\mathcal{H}_{\mu}^{q, t}$ and $\mathcal{P}_{\mu}^{q, t}$ are measures on the family of Borel subsets of $X$. The measure $\mathcal{H}_{\mu}^{q, t}$ is of course a multifractal generalisation of the (centered) Hausdorff measure, whereas $\mathcal{P}_{\mu}^{q, t}$ is a multifractal generalisation of the packing measure. In fact, it is easily seen that the follwing holds for $t \geq 0$,

$$
2^{-t} \mathcal{H}_{\mu}^{0, t} \leq \mathcal{H}^{t} \leq \mathcal{H}_{\mu}^{0, t}, \mathcal{P}^{t}=\mathcal{P}_{\mu}^{0, t}, \overline{\mathcal{P}}^{t}=\overline{\mathcal{P}}_{\mu}^{0, t}
$$


The next result shows that the measures $\mathcal{H}_{\mu}^{q, t}, \mathcal{P}_{\mu}^{q, t}$ and the pre-measure $\overline{\mathcal{P}}_{\mu}^{q, t}$ in the usual way assign a dimension to each subset $E$ of $X$.

Proposition 2.1.1. There exist unique extended real valued numbers $\Delta_{\mu}^{q}(E) \in[-\infty, \infty], \operatorname{Dim}_{\mu}^{q}(E) \in[-\infty, \infty]$ and $\operatorname{dim}_{\mu}^{q}(E) \in[-\infty, \infty]$ such that

$$
\begin{aligned}
& \overline{\mathcal{P}}_{\mu}^{q, t}(E)= \begin{cases}\infty & \text { for } t<\Delta_{\mu}^{q}(E) \\
0 & \text { for } \Delta_{\mu}^{q}(E)<t\end{cases} \\
& \mathcal{P}_{\mu}^{q, t}(E)= \begin{cases}\infty & \text { for } t<\operatorname{Dim}_{\mu}^{q}(E) \\
0 & \text { for } \operatorname{Dim}_{\mu}^{q}(E)<t\end{cases} \\
& \mathcal{H}_{\mu}^{q, t}(E)= \begin{cases}\infty & \text { for } t<\operatorname{dim}_{\mu}^{q}(E) \\
0 & \text { for } \operatorname{dim}_{\mu}^{q}(E)<t .\end{cases}
\end{aligned}
$$

Proof. See [O11, Proposition 1.1].

The number $\operatorname{dim}_{\mu}^{q}(E)$ is an obvious multifractal analogue of the Hausdorff dimension $\operatorname{dim}(E)$ of $E$ whereas $\operatorname{Dim}_{\mu}^{q}(E)$ and $\Delta_{\mu}^{q}(E)$ are obvious multifractal analogues of the packing dimension $\operatorname{Dim}(E)$ and the logarithmic index $\Delta(E)$ of $E$ respectively. In fact, it follows immediately from the definitions that

$$
\operatorname{dim}(E)=\operatorname{dim}_{\mu}^{0}(E), \quad \operatorname{Dim}(E)=\operatorname{Dim}_{\mu}^{0}(E), \Delta(E)=\Delta_{\mu}^{0}(E) .
$$

Next we define multifractal dimension functions $b_{\mu}, B_{\mu}, \Lambda_{\mu}: \mathbb{R} \rightarrow[-\infty, \infty]$ by

$$
b_{\mu}(q)=\operatorname{dim}_{\mu}^{q}(\operatorname{supp} \mu), \quad B_{\mu}(q)=\operatorname{Dim}_{\mu}^{q}(\operatorname{supp} \mu), \quad \Lambda_{\mu}(q)=\Delta_{\mu}^{q}(\operatorname{supp} \mu) .
$$

We will now give a brief list of some of the most important properties of the measures $\mathcal{H}_{\mu}^{q, t}$ and $\mathcal{P}_{\mu}^{q, t}$, and the corresponding dimension functions. The reader is referred to Olsen [O11] for a detailed study of the measure $\mathcal{H}_{\mu}^{q, t}$ and $\mathcal{P}_{\mu}^{q, t}$, and the dimension functions $b_{\mu}, B_{\mu}$ and $\Lambda_{\mu}$. For $\mu \in \mathcal{P}(X)$ and $a>1$ write $T_{a}(\mu)=\lim \sup _{r \searrow 0}\left(\sup _{x \in \operatorname{supp} \mu} \frac{\mu B(x, a r)}{\mu B(x, r)}\right)$ and define the family $\mathcal{P}_{F}(X)$ of Federer probability measures on $X$ by $\mathcal{P}_{F}(X)=\left\{\mu \in \mathcal{P}(X) \mid T_{a}(\mu)<\right.$ $\infty$ for some $a>1\}$. It follows from [O11] that the definition of $\mathcal{P}_{F}(X)$ is independent of the number $a>1$, i.e. $T_{a}(\mu)<\infty$ for all $a>1$ if and only if $T_{a}(\mu)<\infty$ for some $a>1$.

Proposition 2.1.2. Let $\mu \in \mathcal{P}\left(\mathbb{R}^{d}\right)$ and $q, t \in \mathbb{R}$. Then:

i) $\mathcal{H}_{\mu}^{q, t} \leq \mathcal{P}_{\mu}^{q, t}$ for $\mu \in \mathcal{P}_{F}\left(\mathbb{R}^{d}\right)$, and $\mathcal{P}_{\mu}^{q, t} \leq \overline{\mathcal{P}}_{\mu}^{q, t}$ for $\mu \in \mathcal{P}\left(\mathbb{R}^{d}\right)$. 
ii) $\operatorname{dim}_{\mu}^{q} \leq \operatorname{Dim}_{\mu}^{q} \leq \Delta_{\mu}^{q}$, in particular $b_{\mu} \leq B_{\mu} \leq \Lambda_{\mu}$.

iii) $b_{\mu}$ is decreasing, and $B_{\mu}$ and $\Lambda_{\mu}$ are convex and decreasing.

Proof. See [Ol1].

Write

$$
\underline{a}_{\mu}=\sup _{0<q}-\frac{b_{\mu}(q)}{q}, \quad \bar{a}_{\mu}=\inf _{q<0}-\frac{b_{\mu}(q)}{q} .
$$

For a real valued function $f: \mathbb{R} \rightarrow \mathbb{R}$ we define the Legendre transform $f^{*}: \mathbb{R} \rightarrow[-\infty, \infty]$ of $f$ by

$$
f^{*}(x)=\inf _{y}(x y+f(y)) .
$$

Theorem 2.1.3. Let $\mu \in \mathcal{P}\left(\mathbb{R}^{d}\right)$. Then:

i) $\Delta_{\mu}(\alpha)=\varnothing$ for $\alpha \in \mathbb{R}_{+} \backslash\left[\underline{a}_{\mu}, \bar{a}_{\mu}\right]$.

ii) $f_{\mu}(\alpha) \leq b_{\mu}^{*}(\alpha)$ for $\left.\alpha \in\right] \underline{a}_{\mu}, \bar{a}_{\mu}[$.

iii) $F_{\mu}(\alpha) \leq B_{\mu}^{*}(\alpha)$ for $\left.\alpha \in\right] \underline{a}_{\mu}, \bar{a}_{\mu}[$.

Proof. See [Ol1].

Theorem 2.1.4. Let $\mu \in \mathcal{P}\left(\mathbb{R}^{d}\right)$, and $\alpha \geq 0, \delta>0$ and $q, t \in \mathbb{R}$ with $0<\alpha q+t$. Then

i) $\mathcal{H}_{\mu}^{q, t}\left(\Delta_{\mu}(\alpha)\right) \leq 2^{t} \mathcal{H}^{\alpha q+t-\delta}\left(\Delta_{\mu}(\alpha)\right)$.

ii) $\mathcal{P}_{\mu}^{q, t}\left(\Delta_{\mu}(\alpha)\right) \leq 2^{-\alpha q+\delta} \mathcal{P}^{\alpha q+t-\delta}\left(\Delta_{\mu}(\alpha)\right)$.

Proof. See [011].

\subsection{Multifractal box dimensions.}

We begin by recalling the definition of the upper and lower box-dimension. Let $E \subseteq \mathbb{R}^{d}$ be a bounded set and $N_{\delta}(E)$ denote the largest number of disjoint balls of radius $\delta$ with centres in $E$. Then the lower and upper boxdimension of $E$ are defined as

$$
\underline{C}(E)=\liminf _{\delta \searrow 0} \frac{\log N_{\delta}(E)}{-\log \delta}, \quad \bar{C}(E)=\limsup _{\delta \searrow 0} \frac{\log N_{\delta}(E)}{-\log \delta} .
$$

If $\bar{C}(E)=\underline{C}(E)$ we refer to the common value as the box-dimension and denote it by $C(E)$. The reader is referred to $[\mathbf{F a 2}]$ for more information about box-dimensions. We will now define multifractal box-dimensions. Multifractal box dimensions were introduced for example by Falconer [Fa2, p. 225], 
Olsen [Ol1, Ol2], Riedi [Ri1] and Strichartz [St]. Here we follow the approach in Olsen [Ol1]. Let $\mu \in \mathcal{P}\left(\mathbb{R}^{d}\right)$ and $q \in \mathbb{R}$. For $E \subseteq \mathbb{R}^{d}$ and $\delta>0$ write

$$
S_{\mu, \delta}^{q}(E)=\sup \left\{\sum_{i} \mu\left(B\left(x_{i}, \delta\right)\right)^{q} \mid\left(B\left(x_{i}, \delta\right)\right)_{i \in \mathbb{N}} \text { is a centered packing of } E\right\} .
$$

The upper respectively lower multifractal $q$-box dimension $\bar{C}_{\mu}^{q}(E)$ and $\underline{C}_{\mu}^{q}(E)$ of $E$ (with respect to the measure $\mu$ ) is defined by

$$
\bar{C}_{\mu}^{q}(E)=\limsup _{\delta \searrow 0} \frac{\log S_{\mu, \delta}^{q}(E)}{-\log \delta}, \quad \underline{C}_{\mu}^{q}(E)=\liminf _{\delta \searrow 0} \frac{\log S_{\mu, \delta}^{q}(E)}{-\log \delta} .
$$

If $\bar{C}_{\mu}^{q}(E)=\underline{C}_{\mu}^{q}(E)$ we refer to the common value as the $q$-box dimension of $E$ (with respect to the measure $\mu$ ) and denote it by $C_{\mu}^{q}(E)$. Also observe that $\underline{C}_{\mu}^{0}(E)=\underline{C}(E)$ and $\bar{C}_{\mu}^{0}(E)=\bar{C}(E)$. Now write

$$
\underline{\mathcal{C}}_{\mu}(q)=\underline{C}_{\mu}^{q}(\operatorname{supp} \mu), \quad \overline{\mathcal{C}}_{\mu}(q)=\bar{C}_{\mu}^{q}(\operatorname{supp} \mu), \quad \mathcal{C}_{\mu}(q)=C_{\mu}^{q}(\operatorname{supp} \mu) .
$$

The next theorem is proven in Olsen [O11], Proposition 2.19-Proposition 2.23 .

Theorem 2.2.1. $b_{\mu} \leq \underline{\mathcal{C}}_{\mu} \leq \overline{\mathcal{C}}_{\mu}=\Lambda_{\mu}$ for $\mu \in \mathcal{P}_{F}(X)$

\subsection{Generalized Rényi dimensions.}

Generalised Rényi dimensions were introduced for example by the physicists Hentschel \& Procaccia $[\mathbf{H P}]$ and Grassberger \& Procaccia $[\mathbf{G P}]$ in 1983, and later by mathematicians, e.g. Cutler [Cu1], Olsen [Ol1, Ol2], Pesin [Pes1, Pes2] and Strichartz [St]. For $\mu \in \mathcal{P}(X)$ and $q \in \mathbb{R}$ we define the upper and lower generalized Rényi $q$-dimensions of $\mu$ by

$$
\begin{array}{ll}
\bar{D}_{\mu}^{q}=\limsup _{r \searrow 0} \frac{\log \left(\int_{\operatorname{supp} \mu} \mu(B(x, r))^{q} d \mu(x)\right)}{q \log r} & \text { for } q \neq 0 \\
\bar{D}_{\mu}^{0}=\limsup _{r \searrow 0} \frac{\int_{\operatorname{supp} \mu} \log \mu(B(x, r)) d \mu(x)}{\log r} & \text { for } q=0 \\
\underline{D}_{\mu}^{q}=\liminf _{r \searrow 0} \frac{\log \left(\int_{\operatorname{supp} \mu} \mu(B(x, r))^{q} d \mu(x)\right)}{q \log r} & \text { for } q \neq 0 \\
\underline{D}_{\mu}^{0}=\liminf _{r \searrow 0} \frac{\int_{\operatorname{supp} \mu} \log \mu(B(x, r)) d \mu(x)}{\log r} & \text { for } q=0 .
\end{array}
$$


If $\bar{D}_{\mu}^{q}$ and $\underline{D}_{\mu}^{q}$ coincide, we write $D_{\mu}^{q}$ for the common value. Finally define $\overline{\mathcal{D}}_{\mu}, \underline{\mathcal{D}}_{\mu}: \mathbb{R} \rightarrow[-\infty, \infty]$ by

$$
\overline{\mathcal{D}}_{\mu}(q)=(1-q) \bar{D}_{\mu}^{q-1}, \quad \underline{\mathcal{D}}_{\mu}=(1-q) \underline{D}_{\mu}^{q-1} .
$$

Observe that $\underline{\mathcal{D}}_{\mu}(q) \leq \overline{\mathcal{D}}_{\mu}(q)$ for $q \leq 1$, but $\underline{\mathcal{D}}_{\mu}(q) \geq \overline{\mathcal{D}}_{\mu}(q)$ for $1<q$. If $\underline{\mathcal{D}}_{\mu}(q)=\overline{\mathcal{D}}_{\mu}(q)$, we write $\mathcal{D}_{\mu}(q)$ for the common value. The next result was proved in [O11, Theorem 2.24].

Theorem 2.3.1. $\Lambda_{\mu}=\underline{\mathcal{D}}_{\mu} \vee \overline{\mathcal{D}}_{\mu}$ for $\mu \in \mathcal{P}_{F}\left(\mathbb{R}^{d}\right)$.

\subsection{Self-affine Sierpinski sponges.}

Let $d \in \mathbb{N}$ and $1<n_{1} \leq n_{2} \leq \cdots \leq n_{d}$ be integers. Let $I \subseteq \prod_{l=1}^{d}\{0, \ldots$, $\left.n_{l}-1\right\}$. For $\mathbf{i}=\left(i_{1}, \ldots, i_{d}\right) \in I$ define affine maps by $S_{\mathbf{i}}:[0,1]^{d} \rightarrow[0,1]^{d}$ by

$$
S_{\mathbf{i}}\left(x_{1}, \ldots, x_{d}\right)=\left(\frac{1}{n_{1}} x_{1}+\frac{i_{1}}{n_{1}}, \ldots, \frac{1}{n_{d}} x_{d}+\frac{i_{d}}{n_{d}}\right) .
$$

It follows from Hutchinson $[\mathbf{H u}]$ (c.f. also [Fa2]) that there exists a unique non-empty compact set $K$ satisfying

$$
K=\bigcup_{\mathbf{i} \in I} S_{\mathbf{i}}(K)
$$

The set $K$ is called the invariant self-affine set associated with $\left(S_{\mathbf{i}}\right)_{\mathbf{i} \in I}$. Following Kenyon \& Peres $[\mathbf{K P}]$ we call $K$ a Sierpinski Sponge (for $d=2$ we call $K$ a Sierpinski Carpet rather than a Sierpinski Sponge).

The set $K$ can also be constructed in the following way. Let $\Sigma=I^{\mathbb{N}}$, $\Sigma^{(n)}=I^{n}$ for $n \in \mathbb{N}$ and $\Sigma^{(*)}=\cup_{n} \Sigma^{(n)}$. For $\alpha=\left(\mathbf{i}_{1}, \ldots, \mathbf{i}_{n}\right) \in \Sigma^{(*)}$ we write $[\alpha]=\left\{\omega=\left(\mathbf{j}_{1}, \mathbf{j}_{2}, \ldots\right) \in \Sigma \mid \mathbf{i}_{1}=\mathbf{j}_{1}, \ldots, \mathbf{i}_{n}=\mathbf{j}_{n}\right\}, S_{\alpha}=S_{\mathbf{i}_{1}} \circ \cdots \circ S_{\mathbf{i}_{n}}$ and $K_{\alpha}=S_{\alpha}(K)$. For each $\omega=\left(\mathbf{i}_{1}, \mathbf{i}_{2}, \ldots\right) \in \Sigma$ and $n \in \mathbb{N}$ we write $\omega \mid n=\left(\mathbf{i}_{1}, \ldots, \mathbf{i}_{n}\right)$. For each $\omega \in \Sigma,\left(S_{\omega \mid n}\left([0,1]^{d}\right)\right)_{n \in \mathbb{N}}$ is a decreasing sequence of non-empty compact sets whose diameters tend to 0 , hence $\cap_{n} S_{\omega \mid n}\left([0,1]^{d}\right)$ is a singleton. Now define $\pi: \Sigma \rightarrow[0,1]^{d}$ by $\{\pi(\omega)\}=\bigcap_{n} S_{\omega \mid n}\left([0,1]^{d}\right)$. It follows from Hutchinson $[\mathbf{H u}]$ that $K=\pi(\Sigma)$.

Finally, let $T: \Sigma \rightarrow \Sigma$ denote the shift map, i.e. $T\left(\mathbf{i}_{1}, \mathbf{i}_{2}, \ldots\right)=\left(\mathbf{i}_{2}, \mathbf{i}_{3}, \ldots\right)$.

\subsection{Self-affine measures.}

Let $\left(p_{\mathbf{i}}\right)_{\mathbf{i} \in I}$ be a probability vector, i.e. $\left.\left.p_{\mathbf{i}} \in\right] 0,1\right]$ and $\sum_{\mathbf{i}} p_{\mathbf{i}}=1$. Let

$$
\tilde{\mu}:=\prod_{\mathbb{N}}\left(\sum_{\mathbf{i} \in I} p_{\mathbf{i}} \delta_{\mathbf{i}}\right)
$$


(here $\delta_{\mathbf{i}}$ denotes the Dirac measure concentrated at $\mathbf{i}$ ). Define $\mu \in \mathcal{P}(K)$ by

$$
\mu=\tilde{\mu} \circ \pi^{-1} .
$$

The measure $\mu$ is called the self-affine measure associated with $\left(\left(S_{\mathbf{i}}\right)_{\mathbf{i} \in I},\left(p_{\mathbf{i}}\right)_{\mathbf{i} \in I}\right)$. It is easily seen that $\operatorname{supp} \mu=K$. The purpose of this paper is to study the multifractal structure of $\mu$ using the formalism introduced in [Ol1].

Remark. The interesting case is, of course, the case where at least one of the inequalities $n_{1} \leq n_{2} \leq \cdots \leq n_{d}$ is strict. If $n_{1}=n_{2}=\cdots=n_{d}$ then all the maps $S_{\mathbf{i}}$ are similarities, and the multifractal structure of $\mu$ follows immediately from the (substantially more general) theorems in Cawley \& Mauldin [CM], Edgar \& Mauldin [EM] and Olsen [O11] (provided that a certain disjointness condition is satisfied).

\section{Two Auxiliary Functions.}

In this section we introduce and study two fundamental auxiliary functions $\beta$ and $\gamma$. All the main results in Section 4 will be formulated in terms of these functions; in particular, we have

$$
b_{\mu}=\beta, \quad B_{\mu}=\Lambda_{\mu}=\gamma \text { and } f_{\mu}=\beta^{*}
$$

where $\mu$ denotes the self-affine Sierpinski Sponge measure in Section 2.5, c.f. Theorem 4.1.3 and Theorem 4.1.4. The proofs in Sections 3.2, 3.3 and 3.4 are rather lengthy and tedious generalizations of somewhat similar results in [CM, p. 201-206] and will therefore only be briefly sketched or completely omitted.

\subsection{Definition of the auxiliary functions $\beta$ and $\gamma$.}

Fix $l=0, \ldots, d$ and define $\pi_{l}: \mathbb{R}^{d} \rightarrow \mathbb{R}^{l}$ (we put $\mathbb{R}^{0}=\{0\}$ ) by $\pi_{l}\left(x_{1}, \ldots, x_{d}\right)$ $=\left(x_{1}, \ldots, x_{l}\right)$ for $l=1, \ldots, d$, and $\pi_{0}\left(x_{1}, \ldots, x_{d}\right)=0$ for $l=0$. Let

$$
I_{l}=\pi_{l}(I) \text {. }
$$

For $\mathbf{i}=\left(i_{1}, \ldots, i_{d}\right) \in \prod_{l=1}^{d}\left\{0, \ldots, n_{l}-1\right\}$ we define conditional probabilities $p_{1}\left(i_{1}\right), p_{2}\left(i_{2} \mid i_{1}\right), \ldots, p_{d}\left(i_{d} \mid i_{1}, \ldots, i_{d-1}\right)$ by

$$
p_{l}\left(i_{l} \mid i_{1}, \ldots, i_{l-1}\right)= \begin{cases}\frac{\sum_{\substack{\mathbf{j}=\left(j_{1}, \ldots, j_{d}\right) \in I \\ j_{1}=i_{1}, \ldots, j_{l-1}=i_{l-1}, j_{l}=i_{l}}} p_{\mathbf{j}}}{\sum_{\substack{\mathbf{j}=\left(j_{1}, \ldots, j_{d}\right) \in I \\ j_{1}=i_{1}, \ldots, j_{l-1}=i_{l-1}}}} & \text { if }\left(i_{1}, \ldots, i_{l}\right) \in I_{l} \\ 0 & \text { if }\left(i_{1}, \ldots, i_{l}\right) \notin I_{l}\end{cases}
$$


and for $l=1, \ldots, d$ and $\left(i_{1}, \ldots, i_{l}\right) \in I_{l}$ we write

$$
q_{l}\left(i_{1}, \ldots, i_{l}\right)=p_{1}\left(i_{1}\right) p_{2}\left(i_{2} \mid i_{1}\right) \cdots p_{l}\left(i_{l} \mid i_{1}, \ldots, i_{l-1}\right)
$$

For notational convenience write $q_{0}=1$. Define auxiliary functions $\beta_{i_{1} \ldots i_{d}}$, $\beta_{i_{1} \ldots i_{d-1}}, \ldots, \beta_{i_{1} i_{2}}, \beta_{i_{1}}, \beta: \mathbb{R} \rightarrow \mathbb{R}$ inductively as follows:

0) For $q \in \mathbb{R}$ and $\left(i_{1}, \ldots, i_{d}\right) \in I_{d}$ define $\beta_{i_{1} \ldots i_{d}}(q)$ by

$$
\beta_{i_{1} \ldots i_{d}}(q)=0
$$

1) For $q \in \mathbb{R}$ and $\left(i_{1}, \ldots, i_{d-1}\right) \in I_{d-1}$ define $\beta_{i_{1} \ldots i_{d-1}}(q)$ by

$$
\sum_{\substack{i_{d} \\\left(i_{1}, \ldots, i_{d}\right) \in I_{d}}} p_{d}\left(i_{d} \mid i_{1}, \ldots, i_{d-1}\right)^{q} n_{d}^{\beta_{i_{1} \ldots i_{d}}(q)-\beta_{i_{1} \ldots i_{d-1}}(q)}=1
$$

2) For $q \in \mathbb{R}$ and $\left(i_{1}, \ldots, i_{d-2}\right) \in I_{d-2}$ define $\beta_{i_{1} \ldots i_{d-2}}(q)$ by

$$
\sum_{\substack{i_{d-1} \\\left(i_{1}, \ldots, i_{d-1}\right) \in I_{d-1}}} p_{d-1}\left(i_{d-1} \mid i_{1}, \ldots, i_{d-2}\right)^{q} n_{d-1}^{\beta_{i_{1} \ldots i_{d-1}}(q)-\beta_{i_{1} \ldots i_{d-2}}(q)}=1 .
$$

d) For $q \in \mathbb{R}$ define $\beta(q)$ by

$$
\sum_{\substack{i_{1} \\ i_{1} \in I_{1}}} p_{1}\left(i_{1}\right)^{q} n_{1}^{\beta_{i_{1}}(q)-\beta(q)}=1 .
$$

For notational convenience write $\beta_{\varnothing}:=\beta$. The function $\beta$ is clearly differentiable (even real analytic). Now write

$$
\alpha=-\beta^{\prime}
$$

Finally define the auxilairy function $\gamma: \mathbb{R} \rightarrow \mathbb{R}$ by

$$
\gamma(q)=\sum_{l=1}^{d} \frac{1}{\log n_{l}} \log \left(\frac{\sum_{\left(i_{1}, \ldots, i_{l}\right) \in I_{l}} q_{l}\left(i_{1}, \ldots, i_{l}\right)^{q}}{\sum_{\left(i_{1}, \ldots, i_{l-1}\right) \in I_{l-1}} q_{l-1}\left(i_{1}, \ldots, i_{l-1}\right)^{q}}\right),
$$

and put

$$
\zeta=-\gamma^{\prime}
$$




\subsection{Properties of the auxiliary function $\beta$.}

This section investigates the properties of the auxiliary function $\beta$. For $\left(i_{1}, \ldots, i_{d}\right) \in I_{d}$ write

$$
\underline{a}_{i_{1} \ldots i_{d}}=\bar{a}_{i_{1} \ldots i_{d}}=\underline{e}_{i_{1} \ldots i_{d}}=\bar{e}_{i_{1} \ldots i_{d}}=0,
$$

and for $l=1, \ldots, d-1$ and $\left(i_{1}, \ldots, i_{l}\right) \in I_{l}$ we proceed inductively as follows

$$
\begin{gathered}
\gamma_{i_{1} \ldots i_{l}}^{-}=\min _{\substack{i_{l+1} \\
\left(i_{1}, \ldots, i_{l+1}\right) \in I_{l+1}}} p_{l+1}\left(i_{l+1} \mid i_{1}, \ldots, i_{l}\right) n_{l+1}^{-\bar{a}_{i_{1} \ldots i_{l+1}}}, \\
\gamma_{i_{1} \ldots i_{l}}^{+}=\max _{\substack{i_{l+1} \\
\left(i_{1}, \ldots, i_{l+1}\right) \in I_{l+1}}} p_{l+1}\left(i_{l+1} \mid i_{1}, \ldots, i_{l}\right) n_{l+1}^{-\underline{a}_{i_{1} \ldots i_{l+1}}}, \\
\Lambda_{i_{1} \ldots i_{l}}^{-}=\left\{i_{l+1} \mid\left(i_{1}, \ldots, i_{l+1}\right) \in I_{l+1}, p_{l+1}\left(i_{l+1} \mid i_{1}, \ldots, i_{l}\right) n_{l+1}^{-\bar{a}_{i_{1} \ldots i_{l+1}}}=\gamma_{i_{1} \ldots i_{l}}^{-}\right\}, \\
\Lambda_{i_{1} \ldots i_{l}}^{+}=\left\{i_{l+1} \mid\left(i_{1}, \ldots, i_{l+1}\right) \in I_{l+1}, p_{l+1}\left(i_{l+1} \mid i_{1}, \ldots, i_{l}\right) n_{l+1}^{-\underline{a}_{i_{1} \ldots i_{l+1}}}=\gamma_{i_{1} \ldots i_{l}}^{+}\right\}, \\
\underline{a}_{i_{1} \ldots i_{l}}=-\frac{\log \gamma_{i_{1} \ldots i_{l}}^{+}}{\log n_{l+1}}, \bar{a}_{i_{1} \ldots i_{l}}=-\frac{\log \gamma_{i_{1} \ldots i_{l}}^{-}}{\log n_{l+1}}, \\
\underline{e}_{i_{1} \ldots i_{l}}=\frac{\log \left(\sum_{i_{l+1} \in \Lambda_{i_{1} \ldots i_{l}}^{-}} n_{l+1}^{\bar{e}_{i_{1} \ldots i_{l+1}}}\right)}{\log n_{l+1}},
\end{gathered}
$$

Finally write

$$
\begin{gathered}
\gamma^{-}=\min _{i_{1} \in I_{1}} p_{1}\left(i_{1}\right) n_{1}^{-\bar{a}_{i_{1}}}, \gamma^{+}=\max _{i_{1} \in I_{1}} p_{1}\left(i_{1}\right) n_{1}^{-\underline{a}_{i_{1}}}, \\
\Lambda^{-}=\left\{i_{1} \mid i_{1} \in I_{1}, \quad p_{1}\left(i_{1}\right) n_{1}^{-\bar{a}_{i_{1}}}=\gamma^{-}\right\}, \\
\Lambda^{+}=\left\{i_{1} \mid i_{1} \in I_{1}, p_{1}\left(i_{1}\right) n_{1}^{-\underline{a}_{i_{1}}}=\gamma^{+}\right\}, \\
\underline{a}=-\frac{\log \gamma^{+}}{\log n_{1}}, \bar{a}=-\frac{\log \gamma^{-}}{\log n_{1}}, \\
\underline{e}=\frac{\log \left(\sum_{i_{1} \in \Lambda^{+}} n_{1}^{\underline{e}_{i_{1}}}\right)}{\log n_{1}}, \bar{e}=\frac{\log \left(\sum_{i_{1} \in \Lambda^{-}} n_{1}^{\bar{e}_{i_{1}}}\right)}{\log n_{1}},
\end{gathered}
$$

and

$$
s_{i_{1} \ldots i_{d}}=\beta_{i_{1} \ldots i_{d}}(0), \ldots, s_{i_{1}}=\beta_{i_{1}}(0), s=\beta(0) .
$$

For notational convenience define $s_{\varnothing}=s$. We now consider the following two cases:

$$
\text { Case } 1^{\prime}\left\{\begin{array}{c}
\text { For all } \mathbf{i}=\left(i_{1}, \ldots, i_{d}\right) \in I \\
\quad\left(p_{1}\left(i_{1}\right), p_{2}\left(i_{2} \mid i_{1}\right), \ldots, p_{d}\left(i_{d} \mid i_{1}, \ldots, i_{d-1}\right)\right) \\
=\left(n_{1}^{s_{i_{1}}-s}, n_{2}^{s_{i_{1} i_{2}}-s_{i_{1}}}, \ldots, n_{d}^{s_{i_{1}} \ldots i_{d}}-s_{i_{1} \ldots i_{d-1}}\right) \\
\text { which, by the definition of } s_{i_{1} \ldots i_{l}}, \text { is equivalent to } \\
p_{\mathbf{i}}=n_{d}^{s_{i_{1} \ldots i_{d}}-s_{i_{1} \ldots i_{d-1}}} \ldots n_{2}^{s_{i_{1} i_{2}}-s_{i_{1}}} n_{1}^{s_{i_{1}}-s} .
\end{array}\right.
$$




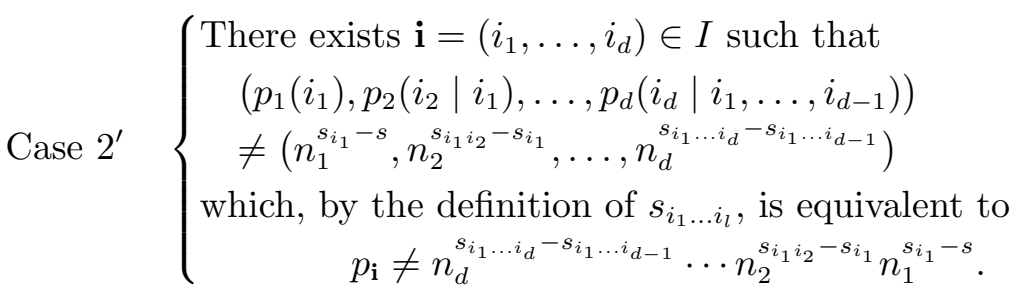

The results below describe the properties of $\beta$.

\section{Proposition 3.2.1.}

i) $\beta$ is convex and strictly decreasing with $\beta(1)=0$.

ii) $\underline{a}, \bar{a}, \underline{e}, \bar{e} \geq 0$ and $\underline{a} \leq \bar{a}$.

iii) $\beta(q)-(-\bar{a} q+\bar{e}) \rightarrow 0$ as $q \rightarrow-\infty$, and the function $q \rightarrow \beta(q)-(-\bar{a} q+\bar{e})$ is increasing.

iv) $\beta(q)-(-\underline{a} q+\underline{e}) \rightarrow 0$ as $q \rightarrow \infty$, and the function $q \rightarrow \beta(q)-(-\underline{a} q+\underline{e})$ is decreasing.

v) $\lim _{q \rightarrow-\infty} \alpha(q)=\bar{a}$ and $\lim _{q \rightarrow \infty} \alpha(q)=\underline{a}$.

Proof. The proof will only be sketched. For each $l=1, \ldots, d-1$, a suitable generalization of the arguments in [CM, p. 201-206] show that, if $\beta_{i_{1} \ldots i_{l+1}}$ and $\alpha_{i_{1} \ldots i_{l+1}}=-\beta_{i_{1} \ldots i_{l+1}}^{\prime}$ satisfy statements i) through $\mathrm{v}$ ) with $\underline{a}, \bar{a}, \underline{e}$ and $\bar{e}$ replaced by $\underline{a}_{i_{1} \ldots i_{l+1}}, \bar{a}_{i_{1} \ldots i_{l+1}}, \underline{e}_{i_{1} \ldots i_{l+1}}$ and $\bar{e}_{i_{1} \ldots i_{l+1}}$, then $\beta_{i_{1} \ldots i_{l}}$ and $\alpha_{i_{1} \ldots i_{l}}=$ $-\beta_{i_{1} \ldots i_{l}}^{\prime}$ satisfy statements i) through v) with $\underline{a}, \bar{a}, \underline{e}$ and $\bar{e}$ replaced by $\underline{a}_{i_{1} \ldots i_{l}}$, $\bar{a}_{i_{1} \ldots i_{l}}, \underline{e}_{i_{1} \ldots i_{l}}$ and $\bar{e}_{i_{1} \ldots i_{l}}$. The result now follows by induction.

\section{Theorem 3.2.2.}

1) If Case $1^{\prime}$ is satisfied, then the following statements hold.

i) $\beta$ is affine, in fact $\beta(q)=s(1-q)$ for all $q$.

ii) $\alpha(q)=-\beta^{\prime}(q)=s$ for all $q$.

iii) $\quad \underline{a}=s=\bar{a}$.

iv) $\beta^{*}(\alpha)=\left\{\begin{array}{ll}s & \text { for } \alpha=s \\ -\infty & \text { for } \alpha \in \mathbb{R} \backslash\{s\}\end{array}\right.$.

2) If Case $2^{\prime}$ is satisfied, then the following statements hold.

i) $\beta$ is strictly convex.

ii) $\alpha(q)=-\beta^{\prime}(q)>0$ for all $q$, and $\alpha$ is strictly decreasing.

iii) $\underline{a}<\bar{a}$.

iv) $\alpha^{\prime}(q)<0$ for all $q$.

v) $\beta^{*}(\alpha)=\left\{\begin{array}{ll}\geq 0 & \text { for } \alpha \in[\underline{a}, \bar{a}] \\ -\infty & \text { for } \alpha \in \mathbb{R} \backslash[\underline{a}, \bar{a}]\end{array}\right.$.

vi) $\quad \beta^{*}$ is strictly concave on $[\underline{a}, \bar{a}]$. 
vii) $\beta^{*}(\alpha(1))=\alpha(1)$ and the straight line with slope 1 and passing through the origin is tangent to the graph of $\beta^{*}$ at the point $\left(\alpha(1), \beta^{*}(\alpha(1))\right)=(\alpha(1), \alpha(1))$.

viii) $\beta^{*}(\underline{a})=\underline{e}, \beta^{*}(\bar{a})=\bar{e}$.

ix) $\sup _{\alpha} \beta^{*}(\alpha)=\beta(0)$ and $\sup _{\alpha} \beta^{*}(\alpha)$ is attained only for $\alpha=\alpha(0)$.

Proof. This result follows from Proposition 3.2.1 using standard results from convex analysis. Again, the reader is referred to [CM, p. 201-206] for proofs of similar results in a simpler setting.

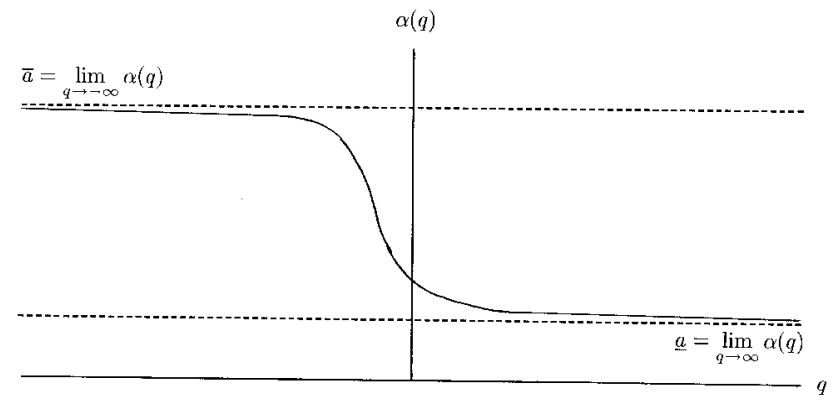

Figure 3.2.1. The typical shape of the graph of $\alpha=-\beta^{\prime}$.

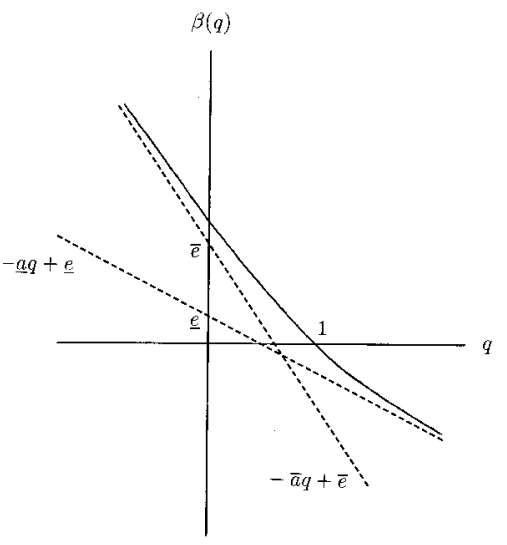

Figures 3.2.2. The typical shape of the graph of $\beta$.

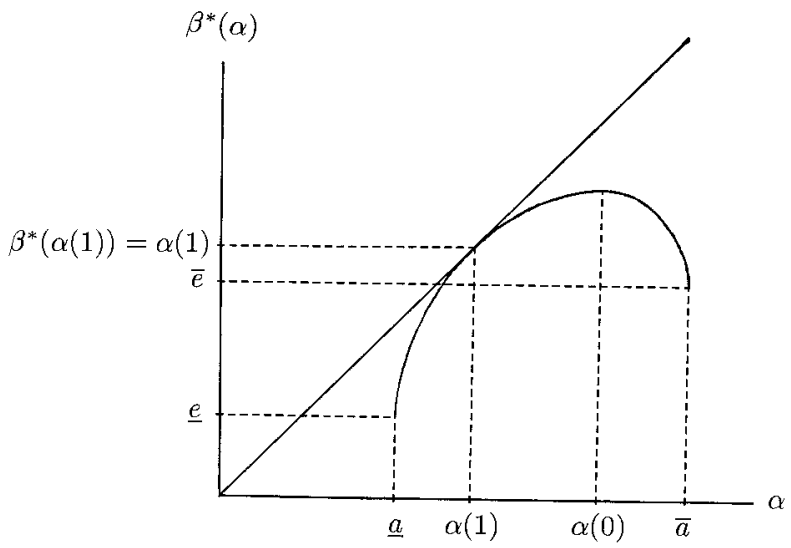

Figure 3.2.3. The typical shape of the graph of $\beta^{*}$.

\subsection{Properties of the auxiliary function $\gamma$.}

This section investigates the properties of the auxiliary function $\gamma$. For $l=1, \ldots, d$ write

$$
q_{l}^{-}=\min _{\left(i_{1}, \ldots, i_{l}\right) \in I_{l}} q_{l}\left(i_{1}, \ldots, i_{l}\right), \quad q_{l}^{+}=\max _{\left(i_{1}, \ldots, i_{l}\right) \in I_{l}} q_{l}\left(i_{1}, \ldots, i_{l}\right)
$$




$$
\begin{aligned}
J_{l}^{-} & =\left\{\left(i_{1}, \ldots, i_{l}\right) \in I_{l} \mid q_{l}\left(i_{1}, \ldots, i_{l}\right)=q_{l}^{-}\right\}, \\
J_{l}^{+} & =\left\{\left(i_{1}, \ldots, i_{l}\right) \in I_{l} \mid q_{l}\left(i_{1}, \ldots, i_{l}\right)=q_{l}^{+}\right\} \\
\underline{A} & =-\sum_{l=1}^{d}\left(\frac{1}{\log n_{l}}-\frac{1}{\log n_{l+1}}\right) \log q_{l}^{+}, \\
\bar{A} & =-\sum_{l=1}^{d}\left(\frac{1}{\log n_{l}}-\frac{1}{\log n_{l+1}}\right) \log q_{l}^{-}, \\
\underline{E} & =\sum_{l=1}^{d}\left(\frac{1}{\log n_{l}}-\frac{1}{\log n_{l+1}}\right) \log \operatorname{card} J_{l}^{+}, \\
\bar{E} & =\sum_{l=1}^{d}\left(\frac{1}{\log n_{l}}-\frac{1}{\log n_{l+1}}\right) \log \operatorname{card} J_{l}^{-} .
\end{aligned}
$$

We now consider the following two cases:

$$
\begin{aligned}
& \text { Case } 1^{\prime \prime}\left\{\begin{array}{c}
\text { For all }\left(i_{1}, \ldots, i_{d}\right) \in I, \\
\left(p_{1}\left(i_{1}\right), p_{2}\left(i_{2} \mid i_{1}\right), \ldots, p_{d}\left(i_{d} \mid i_{1}, \ldots, i_{d-1}\right)\right) \\
=\left(\frac{\operatorname{card} I_{0}}{\operatorname{card} I_{1}}, \frac{\operatorname{card} I_{1}}{\operatorname{card} I_{2}}, \ldots, \frac{\operatorname{card} I_{d-1}}{\operatorname{card} I_{d}}\right) .
\end{array}\right. \\
& \text { Case } 2^{\prime \prime}\left\{\begin{array}{c}
\text { There exists }\left(i_{1}, \ldots, i_{d}\right) \in I \text { such that } \\
\left(p_{1}\left(i_{1}\right), p_{2}\left(i_{2} \mid i_{1}\right), \ldots, p_{d}\left(i_{d} \mid i_{1}, \ldots, i_{d-1}\right)\right) \\
\neq\left(\frac{\operatorname{card} I_{0}}{\operatorname{card} I_{1}}, \frac{\operatorname{card} I_{1}}{\operatorname{card} I_{2}}, \ldots, \frac{\operatorname{card} I_{d-1}}{\operatorname{card} I_{d}}\right) .
\end{array}\right.
\end{aligned}
$$

The results below decribe the properties of $\gamma$. The proofs (which use Hölder's inequality, implicit differentiation and standard results from convex analysis) are elementary albeit rather lengthy generalizations of the arguments in [CM, p. 201-206] and will therefore be omitted.

\section{Proposition 3.3.1.}

i) $\gamma$ is convex and strictly decreasing with $\gamma(1)=0$.

ii) $\underline{A}, \bar{A}, \underline{E}, \bar{E} \geq 0$ and $\underline{A} \leq \bar{A}$.

iii) $\gamma(q)-(-\bar{A} q+\bar{E}) \rightarrow 0$ as $q \rightarrow-\infty$, and the function $q \rightarrow \gamma(q)-$ $(-\bar{A} q+\bar{E})$ is increasing.

iv) $\gamma(q)-(-\underline{A} q+\underline{E}) \rightarrow 0$ as $q \rightarrow \infty$, and the function $q \rightarrow \gamma(q)-(-\underline{A} q+\underline{E})$ is decreasing.

v) $\lim _{q \rightarrow-\infty} \zeta(q)=\bar{A}$ and $\lim _{q \rightarrow \infty} \zeta(q)=\underline{A}$.

\section{Theorem 3.3.2.}

1) If Case $1^{\prime \prime}$ is satisfied, then the following statements hold.

i) $\gamma$ is affine, in fact $\gamma(q)=\gamma(0)(1-q)$ for all $q$. 
ii) $\zeta(q)=-\gamma^{\prime}(q)=-\gamma(0)$ for all $q$.

iii) $\underline{A}=\gamma(0)=\bar{A}$.

iv) $\gamma^{*}(\alpha)=\left\{\begin{array}{ll}\gamma(0) & \text { for } \alpha=\gamma(0) \\ -\infty & \text { for } \alpha \in \mathbb{R} \backslash\{\gamma(0)\}\end{array}\right.$.

2) If Case $2^{\prime \prime}$ is satisfied, then the following statements hold.

i) $\gamma$ is strictly convex.

ii) $\zeta(q)=-\gamma^{\prime}(q)>0$ for all $q$, and $\zeta$ is strictly decreasing.

iii) $\underline{A}<\bar{A}$.

iv) $\zeta^{\prime}(q)<0$ for all $q$.

v) $\gamma^{*}(\alpha)=\left\{\begin{array}{ll}\geq 0 & \text { for } \alpha \in[\underline{A}, \bar{A}] \\ -\infty & \text { for } \alpha \in \mathbb{R} \backslash[\underline{A}, \bar{A}]\end{array}\right.$.

vi) $\gamma^{*}$ is strictly concave on $[\underline{A}, \bar{A}]$.

vii) $\gamma^{*}(\zeta(1))=\zeta(1)$ and the straight line with slope 1 and passing through the origin is tangent to the graph of $\gamma^{*}$ at the point $\left(\zeta(1), \gamma^{*}(\zeta(1))\right)=$ $(\zeta(1), \zeta(1))$.

viii) $\gamma^{*}(\underline{A})=\underline{E}, \gamma^{*}(\bar{A})=\bar{E}$.

ix) $\sup _{\alpha} \gamma^{*}(\alpha)=\gamma(0)$ and $\sup _{\alpha} \gamma^{*}(\alpha)$ is attained only for $\alpha=\zeta(0)$.

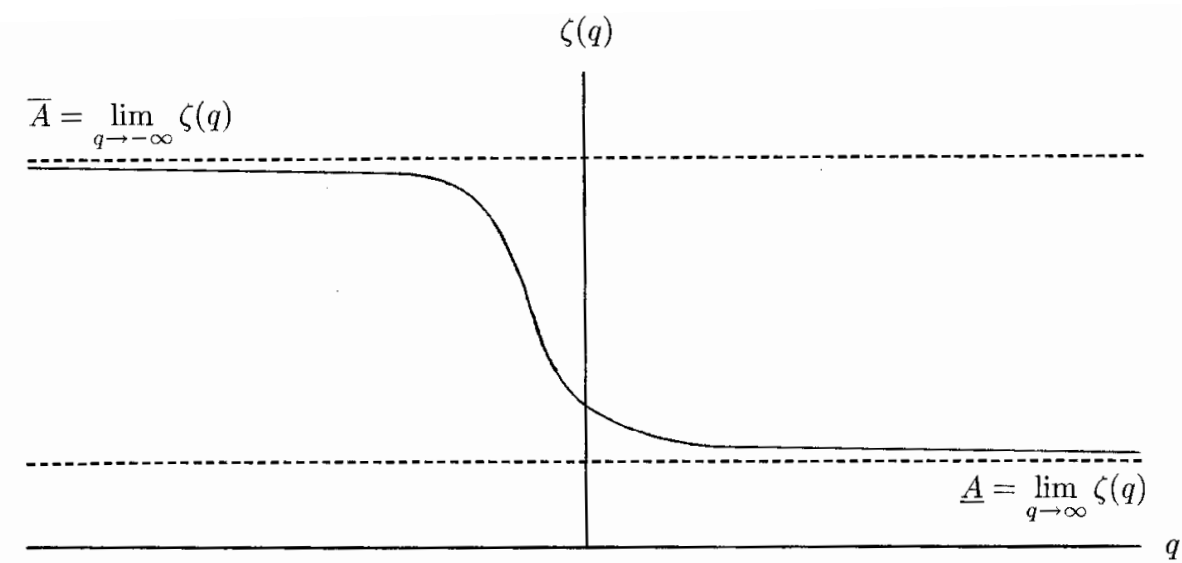

Figure 3.3.1. The typical shape of the graph of $\zeta=-\gamma^{\prime}$. 


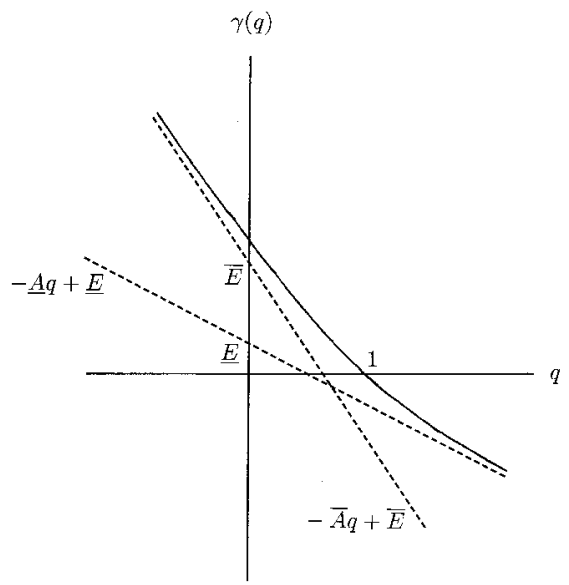

Figure 3.3.2. The typical shape of the graph of $\gamma$.

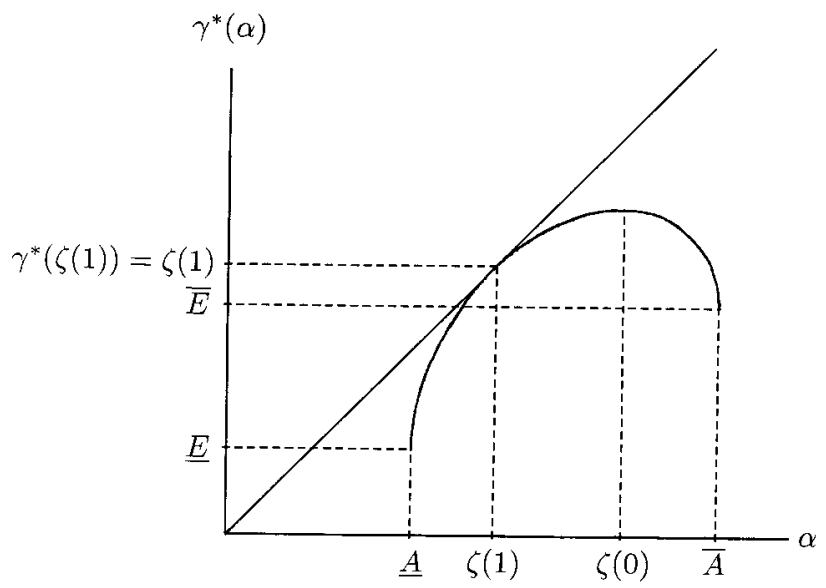

Figure 3.3.3. The typical shape of the graph of $\gamma^{*}$.

\subsection{The relationship between the functions $\beta$ and $\gamma$.}

In this section we investigate the relationship between the functions $\beta$ and $\gamma$.

Theorem 3.4.1. Let $q \in \mathbb{R}$.

i) $\underline{A} \leq \underline{a} \leq \bar{a} \leq \bar{A}$.

ii) $\beta \leq \gamma$.

iii) The following two statements are equivalent.

(1) $\beta(q)=\gamma(q)$.

(2) For each $l=1, \ldots, d-1, n_{l}=n_{l+1}$ or $\beta_{i_{1} \ldots i_{l}}(q)=\beta_{j_{1} \ldots j_{l}}(q)$ for all $\left(i_{1}, \ldots, i_{l}\right),\left(j_{1}, \ldots, j_{l}\right) \in I_{l}$.

iv) If $\beta(q)=\gamma(q)$ then $\alpha(q)=\zeta(q)$ and $\beta^{*}(\alpha(q))=\gamma^{*}(\zeta(q))$. In particular $\alpha(1)=\zeta(1)$.

v) $\beta^{*}(\alpha(q))=q \alpha(q)+\beta(q)$ and $\gamma^{*}(\zeta(q))=q \zeta(q)+\gamma(q)$.

Proof. ii) - iii) Fix $q \in \mathbb{R}, l=1, \ldots, d-1$ and write $\alpha_{l}=\frac{\log n_{l}}{\log n_{l+1}}$. It follows from Jensen's inequality that,

$$
\begin{aligned}
& \sum_{\left(i_{1}, \ldots, i_{l-1}\right) \in I_{l-1}} q_{l-1}\left(i_{1}, \ldots, i_{l-1}\right)^{q} n_{l}^{\beta_{i_{1} \ldots i_{l-1}}(q)} \\
= & \sum_{\left(i_{1}, \ldots, i_{l-1}\right) \in I_{l-1}} q_{l-1}\left(i_{1}, \ldots, i_{l-1}\right)^{q} \sum_{\substack{i_{l} \\
\left(i_{1}, \ldots, i_{l}\right) \in I_{l}}} p_{l}\left(i_{l} \mid i_{1}, \ldots, i_{l-1}\right)^{q} n_{l}^{\beta_{i_{1}} \ldots i_{l}(q)}
\end{aligned}
$$




$$
\begin{aligned}
& =\sum_{\left(i_{1}, \ldots, i_{l}\right) \in I_{l}} q_{l}\left(i_{1}, \ldots, i_{l}\right)^{q}\left(n_{l+1}^{\beta_{i_{1} \ldots i_{l}}(q)}\right)^{\alpha_{l}} \\
& \leq\left(\sum_{\left(i_{1}, \ldots, i_{l}\right) \in I_{l}} q_{l}\left(i_{1}, \ldots, i_{l}\right)^{q}\right)^{1-\alpha_{l}}\left(\sum_{\left(i_{1}, \ldots, i_{l}\right) \in I_{l}} q_{l}\left(i_{1}, \ldots, i_{l}\right)^{q} n_{l+1}^{\beta_{i_{1} \ldots i_{l}}(q)}\right)^{\alpha_{l}}
\end{aligned}
$$

and that,

$$
\text { equality holds in (3.2) if and only if } n_{l}=n_{l+1} \text { or }
$$

$$
\beta_{i_{1} \ldots i_{l}}(q)=\beta_{j_{1} \ldots j_{l}}(q) \text { for all }\left(i_{1}, \ldots, i_{l}\right),\left(j_{1}, \ldots, j_{l}\right) \in I_{l} \text {. }
$$

It follows from repeated application of (3.2) that,

$$
\begin{aligned}
\beta(q) \leq & \left(\frac{1}{\log n_{1}}-\frac{1}{\log n_{2}}\right) \log \left(\sum_{i_{1} \in I_{1}} q_{1}\left(i_{1}\right)^{q}\right) \\
& +\frac{1}{\log n_{2}} \log \left(\sum_{i_{1} \in I_{1}} q_{1}\left(i_{1}\right)^{q} n_{2}^{\beta_{i_{1}}(q)}\right) \\
\leq & \sum_{l=1}^{2}\left(\frac{1}{\log n_{l}}-\frac{1}{\log n_{l+1}}\right) \log \left(\sum_{\left(i_{1}, \ldots, i_{l}\right) \in I_{l}} q_{l}\left(i_{1}, \ldots, i_{l}\right)^{q}\right) \\
& +\frac{1}{\log n_{3}} \log \left(\sum_{\left(i_{1}, i_{2}\right) \in I_{2}} q_{2}\left(i_{1}, i_{2}\right)^{q} n_{3}^{\beta_{i_{1} i_{2}}(q)}\right) \\
& \vdots \\
\leq & \sum_{l=1}^{d-1}\left(\frac{1}{\log n_{l}}-\frac{1}{\log n_{l+1}}\right) \log \left(\sum_{\left(i_{1}, \ldots, i_{l}\right) \in I_{l}} q_{l}\left(i_{1}, \ldots, i_{l}\right)^{q}\right) \\
& +\frac{1}{\log n_{d}} \log \left(\sum_{\left(i_{1}, \ldots, i_{l}\right) \in I_{d}} q_{d}\left(i_{1}, \ldots, i_{d}\right)^{q}\right) \\
= & \gamma(q) .
\end{aligned}
$$

Moreover, (3.3) implies that,

equality holds in (3.4) if and only if for each $l=1, \ldots, d-1$,

$n_{l}=n_{l+1}$ or $\beta_{i_{1} \ldots i_{l}}(q)=\beta_{j_{1} \ldots j_{l}}(q)$ for all $\left(i_{1}, \ldots, i_{l}\right),\left(j_{1}, \ldots, j_{l}\right) \in I_{l}$.

This completes the proof of ii) and iii).

i) It follows from Proposition 3.2.1 and Proposition 3.3.1 that $-\underline{a}=$ $\lim _{q \rightarrow \infty} \frac{\beta(q)}{q}$ and $-\underline{A}=\lim _{q \rightarrow \infty} \frac{\gamma(q)}{q}$, and the inequality $\beta \leq \gamma$ therefore implies that $\underline{A} \leq \underline{a}$. Similarly $\bar{a} \leq \bar{A}$. 
iv) Follows from the fact that the functions $\beta$ and $\gamma$ are convex with $\beta \leq \gamma$ and $\beta(q)=\gamma(q)$.

v) Follows immediately from $\left[\mathbf{R V}\right.$, p. 34, Theorem D] since $\alpha=-\beta^{\prime}$ and $\zeta=-\gamma^{\prime}$.

Remark. The inequalities $\underline{A} \leq \underline{a}$ and $\bar{a} \leq \bar{A}$ can be strict; in fact, it may even happen that $\underline{A}<\underline{a}=\bar{a}<\bar{A}$, i.e. $\beta$ is affine whereas $\gamma$ is strictly convex. Indeed, let $d=2, n_{1}=3, n_{2}=5, I=\{(0,0),(0,2),(2,1)\}$ and $\left(p_{00}, p_{02}, p_{21}\right)=\left(\frac{1}{2} \frac{3^{a}}{1+3^{a}}, \frac{1}{2} \frac{3^{a}}{1+3^{a}}, \frac{1}{1+3^{a}}\right)$ with $a=\frac{\log 2}{\log 5}$. An easy calculation now shows that $\underline{A}<\underline{a}=\bar{a}<\bar{A}$.

\section{Statement of Results.}

Notational Remark. From now on, $\mu$ will always denote the self-affine measure defined in Section 2.5.

We begin by introducing regularity condition $\left(\mathrm{I}_{q}\right)$, for $q \in \mathbb{R}$, and separation condition (II). Condition $\left(\mathrm{I}_{q}\right)$ plays an important role in Theorem 4.1.8 regarding the positivity and finiteness of the multifractal Hausdorff measure and the multifractal packing measure, and we will (unfortunately) have to assume the rather strong separation condition (II) in order to compute $b_{\mu}$, $B_{\mu}$ and $f_{\mu}$ - however, we can compute the cylindre spectrum $f_{\mu}^{\text {c }}$ (defined in Equation (4.5) below) without assuming condition (II).

$\left(\mathrm{I}_{q}\right)\left\{\begin{array}{l}\text { For each } l=1, \ldots, d-1, \text { either } n_{l}=n_{l+1}, \text { or } \\ \quad \sum_{i_{l+1}} p_{l+1}\left(i_{l+1} \mid i_{1}, \ldots, i_{l}\right)^{q}=\sum_{\substack{\left.j_{l}, \ldots, i_{l+1}\right) \in I_{l+1} \\ \text { for all }\left(i_{1}, \ldots, i_{l}\right),\left(j_{1}, \ldots, j_{l}\right) \in I_{l} .}} p_{l+1}\left(j_{l+1} \mid j_{1}, \ldots, j_{l}\right)^{q} \\ \text { The above condition is easily seen to be equivalent to the following: } \\ \text { For each } l=1, \ldots, d-1, \text { either } n_{l}=n_{l+1}, \text { or } \\ \beta_{i_{1} \ldots i_{l}}(q)=\beta_{j_{1} \ldots j_{l}}(q) \\ \text { for all }\left(i_{1}, \ldots, i_{l}\right),\left(j_{1}, \ldots, j_{l}\right) \in I_{l} .\end{array}\right.$

(II) $\left\{\begin{array}{l}\text { If } l=1, \ldots, d \text { and }\left(i_{1}, \ldots, i_{d}\right),\left(j_{1}, \ldots, j_{d}\right) \in I \\ \text { with } i_{1}=j_{1}, \ldots, i_{l-1}=j_{l-1}, i_{l} \neq j_{l} \text { then } \\ \left|i_{l}-j_{l}\right|>1 .\end{array}\right.$

For each $r>0$ and $\omega=\left(\mathbf{i}_{1}, \mathbf{i}_{2}, \ldots\right) \in\left(\prod_{l=1}^{d}\left\{0, \ldots, n_{l}-1\right\}\right)^{\mathbb{N}}$ with $\mathbf{i}_{j}=$ $\left(i_{j, 1}, \ldots, i_{j, d}\right)$ we define the approximate cube (or cylinder) $Q(\omega, r)$ with approximate diameter $r$ determined by $\omega$ as follows: choose (unique) integers 
$k_{1}(r), \ldots, k_{d}(r)$ such that

$$
\frac{1}{n_{l}^{k_{l}(r)+1}}<r \leq \frac{1}{n_{l}^{k_{l}(r)}}
$$

for $l=1, \ldots, d$ and put

$$
Q(\omega, r)=\prod_{l=1}^{d}\left[\frac{i_{1, l}}{n_{l}}+\cdots+\frac{i_{k_{l}(r), l}}{n_{l}^{k_{l}(r)}}, \frac{i_{1, l}}{n_{l}}+\cdots+\frac{i_{k_{l}(r), l}}{n_{l}^{k_{l}(r)}}+\frac{1}{n_{l}^{k_{l}(r)}}\right] .
$$

For $\omega \in \Sigma$ we define the upper and lower local approximate cylinder dimension of $\mu$ at $\omega \in \Sigma$ by

$$
\bar{\alpha}_{\mu}^{\mathbf{c}}(\omega)=\limsup _{r \searrow 0} \frac{\log \mu Q(\omega, r)}{\log r}, \quad \underline{\alpha}_{\mu}^{\mathbf{c}}(\omega)=\liminf _{r \searrow 0} \frac{\log \mu Q(\omega, r)}{\log r} .
$$

If $\bar{\alpha}_{\mu}^{\mathbf{c}}(\omega)$ and $\underline{\alpha}_{\mu}^{\mathbf{c}}(\omega)$ agree we refer to the common value as the local approximate cylinder dimension of $\mu$ at $\omega$ and denote it by $\alpha_{\mu}^{\mathbf{c}}(\omega)$. For $\alpha>0$ write

$$
\Delta_{\mu}^{\mathbf{c}}(\alpha)=\pi\left(\left\{\omega \in \Sigma \mid \alpha_{\mu}^{\mathbf{c}}(\omega)=\alpha\right\}\right) .
$$

Finally define cylinder multifractal spectra functions $f_{\mu}^{\mathbf{c}}$ and $F_{\mu}^{\mathbf{c}}$ by

$$
f_{\mu}^{\mathbf{c}}(\alpha)=\operatorname{dim} \Delta_{\mu}^{\mathbf{c}}(\alpha), \quad F_{\mu}^{\mathbf{c}}(\alpha)=\operatorname{Dim} \Delta_{\mu}^{\mathbf{c}}(\alpha)
$$

We will now state our main results. The proofs will be given in Section 6 . We first compute the cylinder Hausdorff spectrum $f_{\mu}^{\mathrm{c}}$ of $\mu$ without assuming condition (II).

\section{Theorem 4.1.1.}

1) If Case $1^{\prime}$ is satisfied then the following statement holds.

$$
f_{\mu}^{\mathbf{c}}(s)=\beta^{*}(s)=s .
$$

2) If Case $2^{\prime}$ is satisfied then the following statement holds.

$f_{\mu}^{\mathbf{c}}(\alpha)=\beta^{*}(\alpha)$ for $\left.\alpha \in\right] \underline{a}, \bar{a}[$.

\section{Corollary 4.1.2.}

i) $\operatorname{dim} K=\beta(0)=s$.

ii) $C(K)=\gamma(0)$.

Next we compute $f_{\mu}, b_{\mu}, B_{\mu}$ and $\Lambda_{\mu}$ assuming separation condition (II).

Theorem 4.1.3. Assume that condition (II) holds. Then

i) $b_{\mu}=\beta$. 
ii) $B_{\mu}=\Lambda_{\mu}=\gamma$.

iii) $C_{\mu}^{q}(\operatorname{supp} \mu)=(1-q) D_{\mu}^{q-1}=\gamma(q)$ for $q \in \mathbb{R}$.

Theorem 4.1.4. Assume that condition (II) holds.

1) If Case $1^{\prime}$ is satisfied then the following statement holds.

i) $f_{\mu}(s)=\beta^{*}(s)=s$.

ii) $\Delta_{\mu}(\alpha)=\varnothing$ for $\alpha \in \mathbb{R}_{+} \backslash\{s\}$.

2) If Case $2^{\prime}$ is satisfied then the following statement holds.

i) $f_{\mu}(\alpha)=\beta^{*}(\alpha)$ for $\left.\alpha \in\right] \underline{a}, \bar{a}[$.

ii) $\Delta_{\mu}(\alpha)=\varnothing$ for $\alpha \in \mathbb{R}_{+} \backslash[\underline{a}, \bar{a}]$.

\section{Remarks.}

(1) Condition (II) is stronger than asserting that dist $\left(S_{\mathbf{i}}\left([0,1]^{d}\right), S_{\mathbf{j}}\left([0,1]^{d}\right)\right)$ $>0$ for all $\mathbf{i}, \mathbf{j} \in I$ with $\mathbf{i} \neq \mathbf{j}$. If e.g. $d=2$ and $\left(i_{1}, i_{2}\right) \in I$ then (II) asserts that $\left(i_{1} \pm 1, j_{2}\right) \notin I$ for any $j_{2}$, i.e. the two immediately adjacent columns to $\left(i_{1}, i_{2}\right)$ do not contain any points of $K$. In particular we see that (II) implies that the support $\operatorname{supp} \mu=K$ of $\mu$ is totally disconnected. Finally, we note that for $d=2$ condition (II) is identical to the "Disjointness Condition" in King $[\mathbf{K i}]$.

(2) Separation condition (II) is unfortunately very strong and it would be very desirable if condition (II) could be omitted. Recently Arbeiter \& Patzschke $[\mathbf{A P}]$ have succeeded in replacing the strong separation condition with the open set condition in their study of random self-similar multifractals, but it it still not known whether condition (II) can be replaced by the open set condition in the self-affine case. However, we believe that condition (II) can be omitted and we therefore make the following conjecture.

Conjecture 4.1.5. The results in Theorem 4.1 .4 remain true even if separation condition (II) is omitted.

(3) The results in Theorem 4.1.4.1).i) and Theorem 4.1.4.2).i) are higher dimensional generalizations of the main result in King $[\mathbf{K i}]$ where it is assumed that $d=2$.

(4) The results in Corollary 4.1.2 were first obtained independently by Bedford $[\mathrm{Be}]$ and McMullen $[\mathrm{McM}]$ for $d=2$, and later by Kenyon \& Peres $[\mathbf{K P}$, Theorem 1.2 and Proposition 1.3] for arbitrary $d$.

(5) If $n_{1}=n_{2}=\cdots=n_{d}=n$, then all the maps $S_{\mathbf{i}}$ are similarities with Lipschitz constant equal to $\frac{1}{n}$, and an easy calculation shows that

$$
\sum_{\mathbf{i} \in I} p_{\mathbf{i}}^{q} n^{-\beta(q)}=1, \quad \sum_{\mathbf{i} \in I} p_{\mathbf{i}}^{q} n^{-\gamma(q)}=1 .
$$


Hence, if $n_{1}=n_{2}=\cdots=n_{d}$, then Theorem 4.1.3 is a special case of Olsen [Ol1, Theorem 5.1], and Theorem 4.1.4 is a special case of Cawley \& Mauldin [CM, Theorem 2.1] and Olsen [Ol1, Theorem 5.1]

(6) It is possible to construct examples such that the intervals $] \underline{A}, \underline{a}[$ and ] $\bar{a}, \bar{A}$ are non-empty, c.f. the remark following Theorem 3.4.1. Furthermore, Theorem 3.3.2 and Theorem 4.1.4 imply that $F_{\mu}(\alpha)=0<B_{\mu}^{*}(\alpha)$ for $\alpha \in$ ]$\underline{A}, \underline{a}[\cup] \bar{a}, \bar{A}\left[\right.$. The mathematical significance of $B_{\mu}^{*}(\alpha)$ for $\left.\alpha \in\right] \underline{A}, \underline{a}[\cup] \bar{a}, \bar{A}[$ is therefore not (in any obvious way) related to the packing spectrum $F_{\mu}(\alpha)$ at $\alpha$. This phenomenon raises the following question.

Question 4.1.6. What is the significance of $B_{\mu}^{*}(\alpha)$ for $\left.\alpha \in\right] \underline{A}, \underline{a}[\cup] \bar{a}, \bar{A}[$ ?

(7) We have not been able to determine the packing spectrum $F_{\mu}$ of $\mu$. However, we believe that $F_{\mu}$ equals $\gamma^{*}$ and make the following conjecture.

\section{Conjecture 4.1.7.}

1) If Case $1^{\prime \prime}$ is satisfied then

$$
F_{\mu}(s)=\gamma^{*}(s)=s .
$$

2) If Case $2^{\prime \prime}$ is satisfied then

$$
\left.F_{\mu}(\alpha)=\gamma^{*}(\alpha) \quad \text { for } \alpha \in\right] \underline{a}, \bar{a}[.
$$

After having determined the multifractal dimensions $b_{\mu}(q), B_{\mu}(q)$ and $\Lambda_{\mu}(q)$ we turn our attention to the multifractal Hausdorff and packing measures, $\mathcal{H}_{\mu}^{q, b_{\mu}(q)}$ and $\mathcal{P}_{\mu}^{q, B_{\mu}(q)}$, at the critical dimensions. We begin by introducing some notation. If $(A, \mathcal{A}, \nu)$ is a measure space and $E \in \mathcal{A}, \nu\llcorner E$ denotes the restriction of $\nu$ to $E$, i.e. $(\nu\llcorner E) F=\nu(E \cap F)$ for all $F \in \mathcal{A}$. Let $X$ be a metric space and $\nu \in \mathcal{P}(X)$. The Hausdorff dimension $\operatorname{dim} \nu$ of $\nu$ is the lower bound of Hausdorff dimensions of sets which contribute to $\nu$, i.e.

$$
\operatorname{dim} \nu=\inf _{\nu(E)>0} \operatorname{dim} E .
$$

The number $\operatorname{dim} \nu$ is a natural measure of the degree of singularity of $\nu$. Similarly we define the packing dimension $\operatorname{Dim} \nu$ of $\nu$ by

$$
\operatorname{Dim} \nu=\inf _{\nu(E)>0} \operatorname{Dim} E
$$

Theorem 4.1.8. Let $q \in \mathbb{R}$ and assume that conditions $\left(\mathrm{I}_{q}\right)$ and (II) hold. Then

i) $0<\mathcal{H}_{\mu}^{q, \beta(q)}(\operatorname{supp} \mu) \leq \mathcal{P}_{\mu}^{q, \beta(q)}(\operatorname{supp} \mu) \leq \overline{\mathcal{P}}_{\mu}^{q, \beta(q)}(\operatorname{supp} \mu)<\infty$.

ii) $\Delta_{\mu}(\alpha(q))$ has full $\mathcal{H}_{\mu}^{q, \beta(q)}\llcorner\operatorname{supp} \mu$ measure. 
iii) $\Delta_{\mu}(\alpha(q))$ has full $\mathcal{P}_{\mu}^{q, \beta(q)}\llcorner\operatorname{supp} \mu$ measure.

iv) $b_{\mu}(q)=B_{\mu}(q)=\Lambda_{\mu}(q)=\beta(q)=\gamma(q)$.

v) $\operatorname{dim} \mathcal{H}_{\mu}^{q, \beta(q)}\left\llcorner\operatorname{supp} \mu=\beta^{*}(\alpha(q))=\operatorname{Dim} \mathcal{H}_{\mu}^{q, \beta(q)}\llcorner\operatorname{supp} \mu\right.$.

vi) $\operatorname{dim} \mathcal{P}_{\mu}^{q, \beta(q)}\left\llcorner\operatorname{supp} \mu=\beta^{*}(\alpha(q))=\operatorname{Dim} \mathcal{P}_{\mu}^{q, \beta(q)}\llcorner\operatorname{supp} \mu\right.$.

\section{Theorem 4.1.9.}

i) $\operatorname{dim} \mu=\operatorname{Dim} \mu=-\sum_{l=1}^{d} \frac{1}{\log n_{l}} \sum_{\left(i_{1}, \ldots, i_{l}\right) \in I_{l}} q_{l}\left(i_{1}, \ldots, i_{l}\right) \log p_{l}\left(i_{l} \mid i_{1}, \ldots\right.$, $\left.i_{l-1}\right)=\alpha(1)=\zeta(1)$.

ii) If condition (II) holds then $\lim _{r \backslash 0} \frac{\log \mu B(x, r)}{\log r}=\alpha$ (1) for $\mu$-a.a. $x \in K$.

\section{Remarks.}

(1) It follows from Theorem 4.1.8 that condition $\left(\mathrm{I}_{q}\right)$ implies that the multifractal Hausdorff measure and the multifractal packing measure are positive and finite. We conjecture that condition $\left(\mathrm{I}_{q}\right)$ is also necessary.

Conjecture 4.1.10. Let $q \in \mathbb{R}$ and assume that condition (II) holds. Then the following statements are equivalent.

i) $\left(\mathrm{I}_{q}\right)$ holds.

ii) $b_{\mu}(q)=B_{\mu}(q)$.

iii) $0<\mathcal{H}_{\mu}^{q, b_{\mu}(q)}(K)<\infty$ and $0<\mathcal{P}_{\mu}^{q, B_{\mu}(q)}(K)<\infty$.

iv) $0<\mathcal{H}_{\mu}^{q, b_{\mu}(q)}(K)<\infty$.

v) $0<\mathcal{P}_{\mu}^{q, B_{\mu}(q)}(K)<\infty$.

Theorem 4.1 .8 shows that i) $\Rightarrow$ iii), and since $b_{\mu}=\beta$ and $B_{\mu}=\gamma$, Theorem 3.4.1 shows that, i) $\Leftrightarrow$ ii). Of course, trivially iii) $\Rightarrow$ iv) and iii) $\Rightarrow \mathrm{v})$. Recalling that $2^{-t} \mathcal{H}_{\mu}^{0, t} \leq \mathcal{H}^{t} \leq \mathcal{H}_{\mu}^{0, t}$ and $\mathcal{P}_{\mu}^{0, t}=\mathcal{P}^{t}$ for $t \geq 0$, we see that Conjecture 4.1.10 for $q=0$ is contained in [KP, Proposition 1.3], [Pe1, Theorem 1] and [Pe2, Theorem 1.1 and Corollary 2.3.(i)] (except for the assumption that condition (II) holds). We also make the following conjecture.

Conjecture 4.1.11. Let $q \in \mathbb{R}$ and assume that condition $\left(\mathrm{I}_{q}\right)$ does not hold, but that condition (II) holds. Then

i) $\mathcal{H}_{\mu}^{q, b_{\mu}(q)}(K)=\infty$.

ii) $\mathcal{P}_{\mu}^{q, B_{\mu}(q)}(K)=\infty$.

As before, recalling that $2^{-t} \mathcal{H}_{\mu}^{0, t} \leq \mathcal{H}^{t} \leq \mathcal{H}_{\mu}^{0, t}$ and $\mathcal{P}_{\mu}^{0, t}=\mathcal{P}^{t}$ for $t \geq$ 0 , we see that Conjecture 4.1 .11 for $q=0$ and $d=2$ is identical to [Pe1, Theorem 1] and [Pe2, Theorem 1.1] (except for the assumption that condition (II) holds). We mention without proof that we can prove a rather 
weak version of Conjecture 4.1.11, viz. if condition (II) is satisfied, then $\mathcal{P}_{\mu}^{q, B_{\mu}(q)}(K)>0$.

(2) For $d=2$ and $n_{1}<n_{2}$, condition $\left(\mathrm{I}_{q}\right)$ states that,

$$
\left(\sum_{\substack{k \\(i, k) \in I}} p_{i, k}\right)^{-q} \sum_{\substack{k \\(i, k) \in I}} p_{i, k}^{q}=\left(\sum_{\substack{k \\(j, k) \in I}} p_{j, k}\right)^{-q} \sum_{\substack{k \\(j, k) \in I}} p_{i, k}^{q} \quad \text { for all } i, j \in I_{1} \text {. }
$$

For $q=0$, condition $(4.7)$ reduces to

$$
\operatorname{card}\{k \mid(i, k) \in I\}=\operatorname{card}\{k \mid(j, k) \in I\} \quad \text { for all } i, j \in I_{1} .
$$

Recalling that $2^{-t} \mathcal{H}_{\mu}^{0, t} \leq \mathcal{H}^{t} \leq \mathcal{H}_{\mu}^{0, t}, \mathcal{P}_{\mu}^{0, t}=\mathcal{P}^{t}$ and $\overline{\mathcal{P}}_{\mu}^{0, t}=\overline{\mathcal{P}}^{t}$ for $t \geq 0$, we see that Theorem 4.1 .8 for $d=2$ and $q=0$ states that if (4.8) is satisfied, then $0<\mathcal{H}^{s}(K) \leq \mathcal{P}^{s}(K) \leq \overline{\mathcal{P}}^{s}(K)<\infty$. This result, for the Hausdorff measure $\mathcal{H}^{s}(K)$, was first shown by McMullen [McM] without assuming condition (II). The corresponding result for the packing measure, $\mathcal{P}^{s}(K)$, was first obtained by Peres [Pe2, Theorem 1.1.(ii)] also without assuming condition (II). Theorem 4.1 .8 can thus be viewed as a natural multifractal generalization of McMullen's and Peres' results on self-affine carpets.

(3) For $q=0$ and $n_{1}<n_{2}<\cdots<n_{d}$, condition $\left(\mathrm{I}_{q}\right)=\left(\mathrm{I}_{0}\right)$ says that,

For all $l=0, \ldots, d-1$ and $\left(i_{1}, \ldots, i_{l}\right),\left(j_{1}, \ldots, j_{l}\right) \in I_{l}$,

$$
\operatorname{card}\left\{i_{l+1} \mid\left(i_{1}, \ldots, i_{l+1}\right) \in I_{l+1}\right\}=\operatorname{card}\left\{j_{l+1} \mid\left(j_{1}, \ldots, j_{l+1}\right) \in I_{l+1}\right\} .
$$

Recalling that $2^{-t} \mathcal{H}_{\mu}^{0, t} \leq \mathcal{H}^{t} \leq \mathcal{H}_{\mu}^{0, t}, \mathcal{P}_{\mu}^{0, t}=\mathcal{P}^{t}$ and $\overline{\mathcal{P}}_{\mu}^{0, t}=\overline{\mathcal{P}}^{t}$ for $t \geq 0$, we see that Theorem 4.1 .8 for $q=0$ states that if (4.9) is satisfied, then $0<\mathcal{H}^{s}(K) \leq \mathcal{P}^{s}(K) \leq \overline{\mathcal{P}}^{s}(K)<\infty$. This result was first obtained by Kenyon \& Peres $[\mathbf{K P}]$ without assuming condition (II). Theorem 4.1 .8 can thus be viewed as a natural multifractal generalization of Kenyon \& Peres' results on self-affine sponges.

(4) Let $q \in \mathbb{R}$ and assume that $b_{\mu}(q)=B_{\mu}(q)$. Are the measures $\mathcal{H}_{\mu}^{q, b_{\mu}(q)}\llcorner$ $\operatorname{supp} \mu$ and $\mathcal{P}_{\mu}^{q, B_{\mu}(q)}\llcorner\operatorname{supp} \mu$ proportional, i.e. does there exists a constant $c_{q}>0$ such that

$$
\mathcal{P}_{\mu}^{q, B_{\mu}(q)}\left\llcorner\operatorname{supp} \mu=c_{q} \mathcal{H}_{\mu}^{q, b_{\mu}(q)}\llcorner\operatorname{supp} \mu ?\right.
$$

Even though it seems rather unlikely that the multifractal Hausdorff measure and the multifractal packing measure are proportional in general, the ratio of the measures $\mathcal{H}_{\mu}^{q, b_{\mu}(q)}$ Lsupp $\mu$ and $\mathcal{P}_{\mu}^{q, B_{\mu}(q)}$ Lsupp $\mu$ might still be bounded. We therefore ask the following question. 
Question 4.1.12. Does there exists a number $0<c_{q}<\infty$ such that

$$
\mathcal{H}_{\mu}^{q, b_{\mu}(q)}\left\llcorner\operatorname{supp} \mu \leq \mathcal{P}_{\mu}^{q, B_{\mu}(q)}\left\llcorner\operatorname{supp} \mu \leq c_{q} \mathcal{H}_{\mu}^{q, b_{\mu}(q)}\llcorner\operatorname{supp} \mu ?\right.\right.
$$

If $n_{1}=\cdots=n_{d}$, then all the maps $S_{\mathbf{i}}$ are similarities, and it follows from [O11, Theorem 5.1] that Equation (4.10) holds.

(5) If $n_{1}=\cdots=n_{d}=n$, then all the maps $S_{\mathrm{i}}$ are similarities with Lipschitz constant equal to $\frac{1}{n}$ and $\mu$ is a self-similar measure. Furthermore $\alpha(1)=\zeta(1)=\frac{\sum_{\mathrm{i}} p_{\mathrm{i}} \log p_{\mathrm{i}}}{\sum_{\mathrm{i}} p_{\mathrm{i}} \log \frac{1}{n}}$, and Theorem 4.1.9 therefore states that $\operatorname{dim} \mu=\operatorname{Dim} \mu=\frac{\sum_{\mathbf{i}} p_{\mathrm{i}} \log p_{\mathrm{i}}}{\sum_{\mathbf{i}} p_{\mathbf{i}} \log \frac{1}{n}}$ and (if in addition condition (II) holds) $\alpha_{\mu}(x)=$

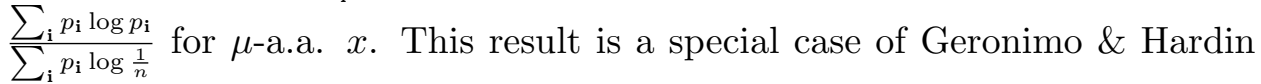
[GH, Theorem 2 and Corollary 1].

\section{An Example.}

Let $d=3, n_{1}=3, n_{2}=5$ and $n_{3}=6$. Put

$$
I=\{(0,0,0),(0,4,0),(2,1,0),(2,3,0),(0,2,1),(0,0,2) \text {, }
$$

$$
(0,2,3),(0,4,4),(2,3,4),(2,1,5)\},
$$

and

$$
\begin{aligned}
\left(p_{000}, p_{040}, p_{210}, p_{230}, p_{021}, p_{002}, p_{023}, p_{044}, p_{234}, p_{215}\right) \\
\quad=\left(\frac{1}{15}, \frac{1}{20}, \frac{1}{15}, \frac{1}{20}, \frac{1}{5}, \frac{1}{10}, \frac{1}{10}, \frac{1}{20}, \frac{1}{5}, \frac{7}{60}\right) .
\end{aligned}
$$

A straightforward calculation shows that

$$
\begin{aligned}
\beta_{00}(q) & =\frac{1}{\log 6} \log \left(\left(\frac{2}{5}\right)^{q}+\left(\frac{3}{5}\right)^{q}\right), \\
\beta_{02}(q) & =\frac{1}{\log 6} \log \left(\left(\frac{2}{3}\right)^{q}+\left(\frac{1}{3}\right)^{q}\right), \quad \beta_{04}(q)=\frac{\log 2}{\log 6}(1-q) \\
\beta_{21}(q) & =\frac{1}{\log 6} \log \left(\left(\frac{4}{11}\right)^{q}+\left(\frac{7}{11}\right)^{q}\right), \quad \beta_{23}(q)=\frac{1}{\log 6} \log \left(\left(\frac{1}{5}\right)^{q}+\left(\frac{4}{5}\right)^{q}\right) \\
\beta_{0}(q) & =\frac{1}{\log 5} \log \left(\left(\frac{5}{17}\right)^{q} 5^{\beta_{00}(q)}+\left(\frac{9}{17}\right)^{q} 5^{\beta_{02}(q)}+\left(\frac{3}{17}\right)^{q} 5^{\beta_{04}(q)}\right) \\
\beta_{2}(q) & =\frac{1}{\log 5} \log \left(\left(\frac{11}{26}\right)^{q} 5^{\beta_{21}(q)}+\left(\frac{15}{26}\right)^{q} 5^{\beta_{23}(q)}\right) \\
\beta(q) & =\frac{1}{\log 3} \log \left(\left(\frac{17}{30}\right)^{q} 3^{\beta_{0}(q)}+\left(\frac{13}{30}\right)^{q} 3^{\beta_{2}(q)}\right) \\
\underline{a} & =\frac{\log \left(\frac{30}{17}\right)}{\log 3}+\frac{\log \left(\frac{17}{9}\right)}{\log 5}+\frac{\log \left(\frac{3}{2}\right)}{\log 6} \approx 1.13846, \\
\bar{a} & =\frac{\log \left(\frac{30}{13}\right)}{\log 3}+\frac{\log \left(\frac{26}{15}\right)}{\log 5}+\frac{\log 5}{\log 6} \approx 2.00119, \underline{e}=\bar{e}=0 .
\end{aligned}
$$


Also

$$
\begin{aligned}
\gamma(q)= & \left(\frac{1}{\log 3}-\frac{1}{\log 5}\right) \log \left(\left(\frac{17}{30}\right)^{q}+\left(\frac{13}{30}\right)^{q}\right) \\
& +\left(\frac{1}{\log 5}-\frac{1}{\log 6}\right) \log \left(\left(\frac{1}{6}\right)^{q}+\left(\frac{3}{10}\right)^{q}+\left(\frac{1}{10}\right)^{q}+\left(\frac{11}{60}\right)^{q}+\left(\frac{1}{4}\right)^{q}\right) \\
& +\frac{1}{\log 6} \log \left(3\left(\frac{1}{20}\right)^{q}+2\left(\frac{1}{15}\right)^{q}+2\left(\frac{1}{10}\right)^{q}+2\left(\frac{1}{5}\right)^{q}+\left(\frac{7}{60}\right)^{q}\right) \\
\underline{A}= & \underline{a}, \bar{A}=\frac{\log \left(\frac{30}{13}\right)}{\log 3}+\frac{\log \left(\frac{13}{3}\right)}{\log 5}+\frac{\log 2}{\log 6} \approx 2.05913, \\
\underline{E}= & \frac{\log 2}{\log 6} \approx 0.386853, \bar{E}=\frac{\log 3}{\log 6} \approx 0.613147 .
\end{aligned}
$$

Observe that $\underline{A}=\underline{a}$ and $\bar{a}<\bar{A}$. An easy calculation shows that condition $\left(\mathrm{I}_{q}\right)$ holds if and only if $q=1$. Below we sketch graphs of $\beta=b_{\mu}, \beta^{*}, \gamma=B_{\mu}$ and $\gamma^{*}$.

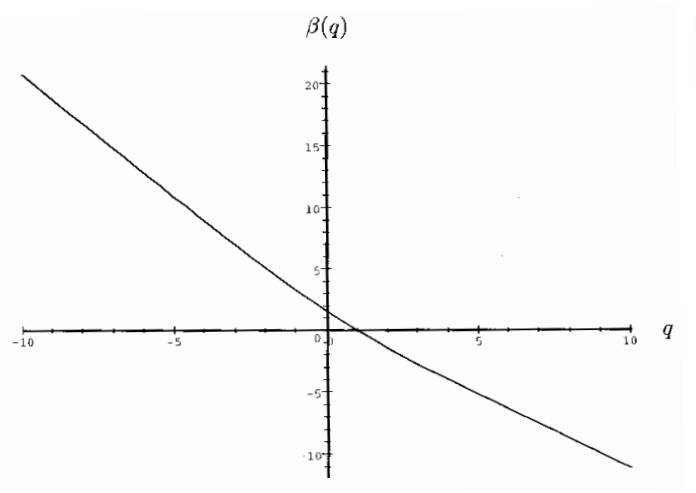

Figure 5.1.1. The graph of $\beta$.

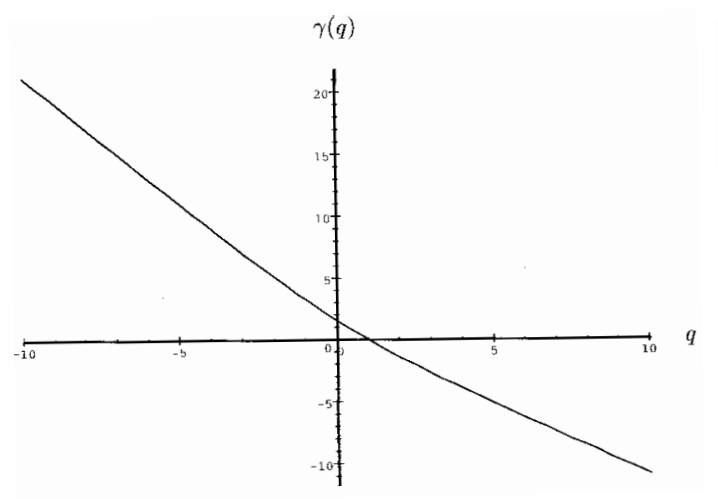

Figure 5.1.3. The graph of $\gamma$.

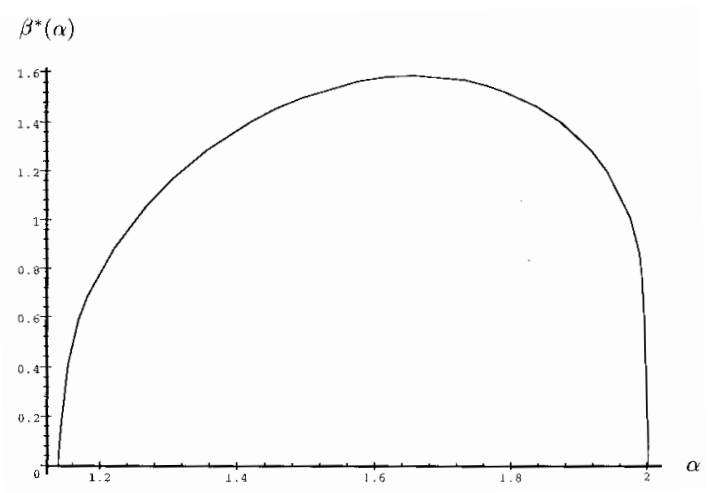

Figure 5.1.2. The graph of $\beta^{*}$.

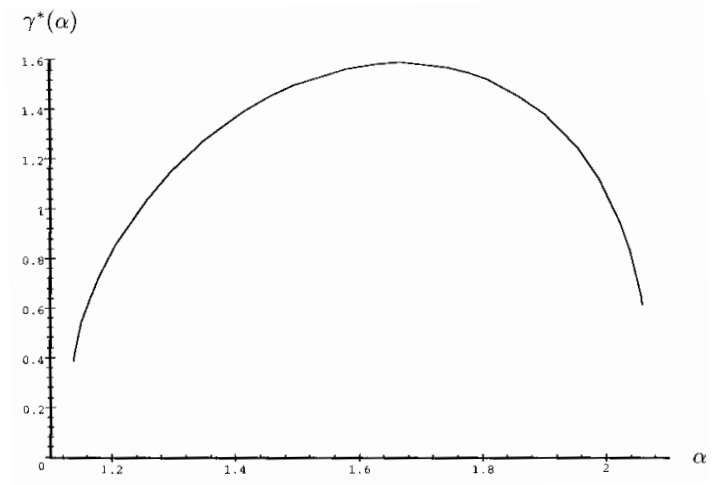

Figure 5.1.4. The graph of $\gamma^{*}$. 

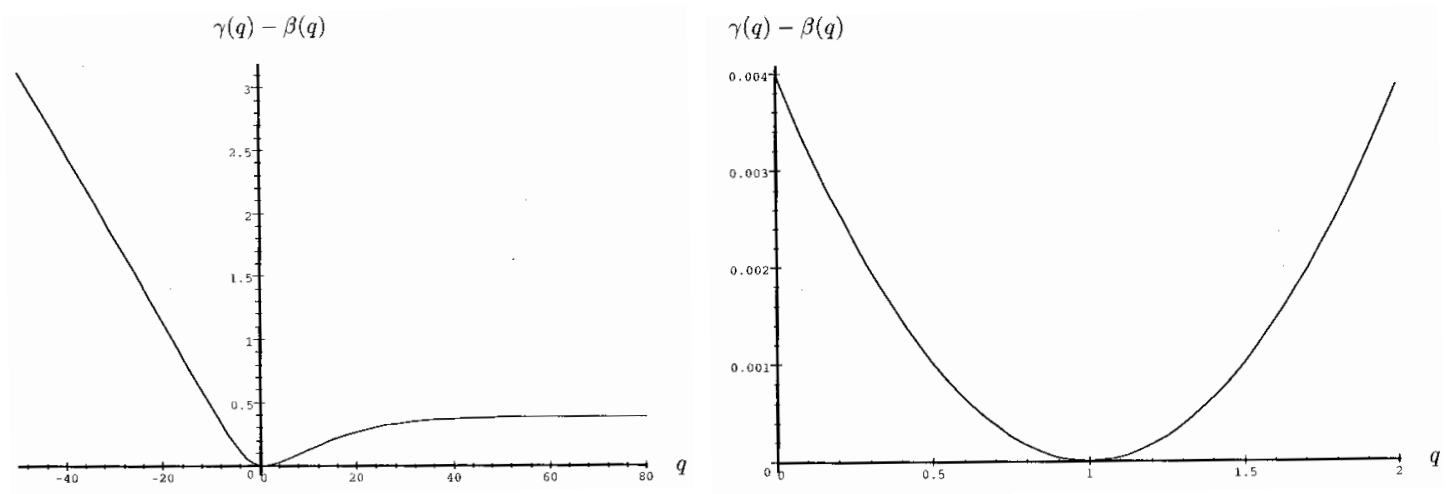

Figure 5.1.5. The graph of $\gamma-\beta$.

\section{Proofs.}

\subsection{Preliminary results.}

For $q \in \mathbb{R}$ and $\mathbf{i}=\left(i_{1}, \ldots, i_{d}\right) \in I$ put

$$
P_{\mathbf{i}}(q)=p_{\mathbf{i}}^{q} n_{d}^{\beta_{i_{1} \ldots i_{d}}(q)-\beta_{i_{1} \ldots i_{d-1}}(q)} n_{d-1}^{\beta_{i_{1} \ldots i_{d-1}}(q)-\beta_{i_{1} \ldots i_{d-2}}(q)} \cdots n^{\beta_{i_{1}}(q)-\beta(q)},
$$

and define conditional probabilities $P_{1}\left(q ; i_{1}\right), P_{2}\left(q ; i_{2} \mid i_{1}\right), \ldots, P_{d}\left(q ; i_{d} \mid i_{1}, \ldots\right.$, $\left.i_{d-1}\right)$ by

$$
P_{l}\left(q ; i_{l} \mid i_{1}, \ldots, i_{l-1}\right)=\left\{\begin{array}{ll}
\frac{\sum_{\substack{\mathbf{j}=\left(j_{1}, \ldots, j_{d}\right) \in I \\
j_{1}=i_{1}, \ldots, j_{l}-1=i_{l-1}, j_{l}=i_{l}}} P_{\mathbf{j}}(q)}{\sum_{\substack{\mathbf{j}=\left(j_{1}, \ldots, j_{d}\right) \in I \\
j_{1}=i_{1}, \ldots, j_{l-1}=i_{l-1}}}} & \text { if }\left(i_{1}, \ldots, i_{l}\right) \in I_{l} . \\
0 & \text { if }\left(i_{1}, \ldots, i_{l}\right) \notin I_{l}
\end{array} .\right.
$$

For $l=1, \ldots, d,\left(i_{1}, \ldots, i_{d}\right) \in \prod_{j=1}^{d}\left\{0, \ldots, n_{j}-1\right\}$ and $\omega=\left(\mathbf{i}_{1}, \mathbf{i}_{2}, \ldots\right) \in \Sigma$ with $\mathbf{i}_{j}=\left(i_{j, 1}, \ldots, i_{j, d}\right)$ write

$$
\begin{aligned}
p_{l}(\omega) & =p_{l}\left(i_{1, l} \mid i_{1,1}, \ldots, i_{1, l-1}\right), \\
P_{l}(q ; \omega) & =P_{d}\left(q ; i_{1, l} \mid i_{1,1}, \ldots, i_{1, l-1}\right), \\
Q_{l}\left(q ; i_{1}, \ldots, i_{l}\right) & =P_{1}\left(q ; i_{1}\right) P_{2}\left(q ; i_{2} \mid i_{1}\right) \cdots P_{l}\left(q ; i_{l} \mid i_{1}, \ldots, i_{l-1}\right) .
\end{aligned}
$$

Put $\alpha_{l}=\frac{\log n_{l}}{\log n_{l+1}}$ for $l=1, \ldots, d-1$. For notational convenience define $\alpha_{0}=1$. For $\tau=\left(\mathbf{i}_{1}, \mathbf{i}_{2}, \ldots, \mathbf{i}_{n}\right) \in \Sigma^{(n)}$ and $\mathbf{i}=\left(i_{1}, \ldots, i_{d}\right) \in I$ write

$$
u_{\mathbf{i}}(q ; l)=n_{l}^{\beta_{i_{1} \ldots i_{l}}(q)}, \quad u_{\tau}(q ; l)=u_{\mathbf{i}_{1}}(q ; l) \cdots u_{\mathbf{i}_{n}}(q ; l) \quad \text { for } l=1, \ldots, d
$$




$$
v_{\mathbf{i}}(q ; l)=n_{l+1}^{\beta_{i_{1} \ldots i_{l}}(q)}, \quad v_{\tau}(q ; l)=v_{\mathbf{i}_{1}}(q ; l) \cdots v_{\mathbf{i}_{n}}(q ; l) \quad \text { for } l=0, \ldots, d-1 .
$$

Observe that $v_{\mathbf{i}}(q ; l)=u_{\mathbf{i}}(q ; l)^{\frac{1}{\alpha_{l}}}$ and $v_{\tau}(q ; l)=u_{\tau}(q ; l)^{\frac{1}{\alpha_{l}}}$ for $l=1, \ldots, d-1$. Also write

$$
a_{n}(q ; \omega, l)=u_{\omega \mid n}(q ; l)^{\alpha_{1} \cdots \alpha_{l-1} \frac{1}{n}}
$$

for $\omega \in \Sigma$ and $n \in \mathbb{N}$. Let $a(q)=\min _{l=1, \ldots, d-1} \min _{\mathbf{i} \in I} u_{\mathbf{i}}(q ; l)^{\alpha_{1} \cdots \alpha_{l-1}}$ and $A(q)=\max _{l=1, \ldots, d-1} \max _{\mathbf{i} \in I} u_{\mathbf{i}}(q ; l)^{\alpha_{1} \cdots \alpha_{l-1}}$, and note that

$$
\begin{aligned}
& 0<a(q) \leq a_{n}(q ; \omega, l) \leq A(q)<\infty \\
& \quad \text { for all } \omega \in \Sigma, n \in \mathbb{N} \text { and } l=1, \ldots, d-1 .
\end{aligned}
$$

The proofs of Lemma 6.1.1 and Lemma 6.1.2 follow easily from the definitions.

Lemma 6.1.1. Let $q \in \mathbb{R}, l=0, \ldots, d-1$ and $\left(i_{1}, \ldots, i_{l}\right) \in I_{l}$. Then

$$
\begin{aligned}
& \sum_{\substack{i_{l+1}, \ldots, i_{d} \\
\left(i_{1}, \ldots, i_{d}\right) \in I}} p_{l+1}\left(i_{l+1} \mid i_{1}, \ldots, i_{l}\right)^{q} \cdots p_{d}\left(i_{d} \mid i_{1}, \ldots, i_{d-1}\right)^{q} \\
& \cdot n_{d}^{\beta_{i_{1} \ldots i_{d}}(q)-\beta_{i_{1} \ldots i_{d-1}}(q)} n_{d-1}^{\beta_{i_{1} \ldots i_{d-1}}(q)-\beta_{i_{1} \ldots i_{d-2}}(q)} \cdots n_{l+1}^{\beta_{i_{1} \ldots i_{l+1}}(q)-\beta_{i_{1} \ldots i_{l}}(q)}=1 .
\end{aligned}
$$

Lemma 6.1.2. Let $q \in \mathbb{R}, l=1, \ldots, d$ and $\left(i_{1}, \ldots, i_{l}\right) \in I_{l}$. Then

$$
\frac{Q_{l}\left(q ; i_{1}, \ldots, i_{l}\right)}{q_{l}\left(i_{1}, \ldots, i_{l}\right)^{q}}=n_{l}^{\beta_{i_{1} \ldots i_{l}}(q)-\beta_{i_{1} \ldots i_{l-1}}(q)} \cdots n_{1}^{\beta_{i_{1}}(q)-\beta(q)} .
$$

Let $q \in \mathbb{R}$. It follows from Lemma 6.1.1 that $\sum_{\mathbf{i} \in I} P_{\mathbf{i}}(q)=1$, and we can thus define a probability measure $\tilde{\mu}^{q}$ on $\Sigma$ by

$$
\tilde{\mu}^{q}=\prod_{\mathbb{N}}\left(\sum_{\mathbf{i} \in I} P_{\mathbf{i}}(q) \delta_{\mathbf{i}}\right) .
$$

Next define $\mu^{q} \in \mathcal{P}(K)$ by

$$
\mu^{q}=\tilde{\mu}^{q} \circ \pi^{-1}
$$

Clearly, since $n_{1} \leq n_{2} \leq \cdots \leq n_{d}$ (recall that $T: \Sigma \rightarrow \Sigma$ denotes the shift map),

$$
\mu(Q(\omega, r))=\prod_{l=1}^{d} \prod_{j=0}^{k_{l}(r)-1} p_{l}\left(T^{j} \omega\right)
$$




$$
\mu^{q}(Q(\omega, r))=\prod_{l=1}^{d} \prod_{j=0}^{k_{l}(r)-1} P_{l}\left(q ; T^{j} \omega\right) .
$$

For $q \in \mathbb{R}, \omega \in \Sigma$ and $r>0$ define the approximate $r$-order $q$-density $D_{r}(q ; \omega)$ of $\mu$ at $\omega$ by

$$
D_{r}(q ; \omega)=\left(\frac{\mu^{q}(Q(\omega, r))}{\mu(Q(\omega, r))^{q}\left(n_{1}^{-k_{1}(r)}\right)^{\beta(q)}}\right)^{\frac{1}{k_{1}(r)}} .
$$

We will prove that $D_{r}(q ; \omega)$ typically is close to 1 for $r$ close to 0 ; specifically, we will prove that $\lim \sup _{r \searrow 0} D_{r}(q ; \omega) \geq 1$ for all $q \in \mathbb{R}$ and for all $\omega \in \Sigma$ (c.f. Proposition 6.1.6), and that $\lim _{r \backslash 0} D_{r}(q ; \omega)=1$ for $\tilde{\mu}^{q}$-a.a. $\omega \in \Sigma$ (c.f. Lemma 6.3.4).

Proposition 6.1.3. Let $q \in \mathbb{R}, \omega \in \Sigma$ and $r>0$. Then

$$
\begin{aligned}
D_{r}(q ; \omega)= & \left(\prod_{\substack{l \\
\alpha_{l} \neq 1}}\left(\frac{a_{k_{l}(r)}(q ; \omega, l)}{a_{k_{l+1}(r)}(q ; \omega, l)}\right)^{\frac{1}{\alpha_{1} \cdots \alpha_{l-1}} \frac{k_{l}(r)}{k_{1}(r)}}\right) \\
& \cdot\left(\prod_{\substack{l \\
\alpha_{l}=1}}\left(a_{k_{l+1}(r)}(q ; \omega, l)^{1-\frac{1}{\alpha_{l}} \frac{k_{l+1}(r)}{k_{l}(r)}}\right)^{\frac{1}{\alpha_{1} \cdots \alpha_{l-1}} \frac{k_{l}(r)}{k_{1}(r)}}\right) .
\end{aligned}
$$

Proof. Let $\omega=\left(\mathbf{i}_{1}, \mathbf{i}_{2}, \ldots\right)$ with $\mathbf{i}_{j}=\left(i_{j, 1}, \ldots, i_{j, d}\right)$. It follows from (6.2) and (6.3) that

$$
\begin{aligned}
& D_{r}(q ; \omega)^{k_{1}(r)} \\
& =\frac{\mu^{q}(Q(\omega, r))}{\mu(Q(\omega, r))^{q}\left(n_{1}^{-k_{1}(r)}\right)^{\beta(q)}} \\
& =\frac{1}{\left(n_{1}^{-k_{1}(r)}\right)^{\beta(q)}} \frac{\prod_{l=1}^{d} \prod_{j=0}^{k_{l}(r)-1} P_{l}\left(q ; T^{j} \omega\right)}{\prod_{l=1}^{d} \prod_{j=0}^{k_{l}(r)-1} p_{l}\left(T^{j} \omega\right)^{q}} \\
& =\frac{1}{\left(n_{1}^{-k_{1}(r)}\right)^{\beta(q)}} \prod_{l=1}^{d} \frac{\prod_{j=1}^{k_{l}(r)} P_{l}\left(q ; i_{j, l} \mid i_{j, 1}, \ldots, i_{j, l-1}\right)}{\prod_{j=1}^{k_{l}(r)} p_{l}\left(i_{j, l} \mid i_{j, 1}, \ldots, i_{j, l-1}\right)^{q}} \\
& =\frac{1}{\left(n_{1}^{-k_{1}(r)}\right)^{\beta(q)}} \frac{\prod_{j=1}^{k_{d}(r)} Q_{d}\left(q ; i_{j, 1}, \ldots, i_{j, d}\right)}{\prod_{j=1}^{k_{d}(r)} q_{d}\left(i_{j, 1}, \ldots, i_{j, d}\right)^{q}}
\end{aligned}
$$




$$
\begin{aligned}
& \cdot \prod_{l=1}^{d-1}\left(\frac{\prod_{j=k_{l+1}(r)+1}^{k_{l}(r)} Q_{l}\left(q ; i_{j, 1}, \ldots, i_{j, l}\right)}{\prod_{j=k_{l+1}(r)+1}^{k_{l}(r)} q_{l}\left(i_{j, 1}, \ldots, i_{j, l}\right)}\right) \\
& =\frac{1}{\left(n_{1}^{-k_{1}(r)}\right)^{\beta(q)}} \quad \frac{\prod_{j=1}^{d} u_{\omega \mid k_{d}(r)}(q ; j)}{\prod_{j=1}^{d} v_{\omega \mid k_{d}(r)}(q ; j-1)} \prod_{l=1}^{d-1}\left(\frac{\prod_{j=1}^{l} \frac{u_{\omega \mid k_{l}(r)}(q ; j)}{u_{\omega \mid k_{l+1}(r)}(q ; j)}}{\prod_{j=1}^{l} \frac{v_{\omega \mid k_{l}(r)}(q ; j-1)}{v_{\omega \mid k_{l+1}(r)}(q ; j-1)}}\right) \\
& =\frac{1}{\left(n_{1}^{-k_{1}(r)}\right)^{\beta(q)}} u_{\omega \mid k_{d}(r)}(q ; d)\left(\prod_{l=1}^{d-1} \frac{u_{\omega \mid k_{l}(r)}(q ; l)}{v_{\omega \mid k_{l}(r)}(q ; l)}\right) \frac{1}{v_{\omega \mid k_{1}(r)}(q ; 0)} \\
& =\prod_{l=1}^{d-1} \frac{u_{\omega \mid k_{l}(r)}(q ; l)}{\left(u_{\omega \mid k_{l+1}(r)}(q ; l)\right)^{\frac{1}{\alpha_{l}}}} \\
& =\left(\prod_{l=1}^{d-1}\left(\frac{a_{k_{l}(r)}(q ; \omega, l)}{a_{k_{l+1}(r)}(q ; \omega, l)} a_{k_{l+1}(r)}(q ; \omega, l)^{1-\frac{1}{\alpha_{l}} \frac{k_{l+1}(r)}{k_{l}(r)}}\right)^{\frac{1}{\alpha_{1} \cdots \alpha_{l-1}} \frac{k_{l}(r)}{k_{1}(r)}}\right)^{k_{1}(r)} .
\end{aligned}
$$

The result follows from the above formula since $k_{l}(r)=k_{l+1}(r)$ for all $l$ with $\alpha_{l}=1$.

Lemma 6.1.4. ([KP, Lemma 4.1]). Let $N \in \mathbb{N}$ and $f_{1}, \ldots, f_{N}: \mathbb{N} \rightarrow \mathbb{R}$ satisfy

$$
\max _{i} \sup _{n \in \mathbb{N}}\left|f_{i}(n+1)-f_{i}(n)\right|<\infty
$$

Let $\left.\alpha_{1}, \ldots, \alpha_{N}, L_{1}, \ldots, L_{N} \in\right] 0, \infty[$. Then

$$
\limsup _{t \rightarrow \infty} \frac{1}{t} \sum_{i=1}^{N}\left(\alpha_{i} f_{i}\left(\left[\frac{t}{L_{i}}\right]\right)-f_{i}\left(\left[\alpha_{i} \frac{t}{L_{i}}\right]\right)\right) \geq 0 .
$$

Proof. See [KP, Lemma 4.1].

Lemma 6.1.5. Let $q \in \mathbb{R}$ and $\omega \in \Sigma$. Then

$$
\limsup _{r \searrow 0} \frac{\prod_{l=1}^{d-1} a_{k_{l}(r)}(q ; \omega, l)}{\prod_{l=1}^{d-1} a_{k_{l+1}(r)}(q ; \omega, l)} \geq 1 .
$$

Proof. Write $\omega=\left(\mathbf{i}_{1}, \mathbf{i}_{2}, \ldots\right)$. For $l=1, \ldots, d-1$ define $f_{l}: \mathbb{N} \rightarrow \mathbb{R}$ by $f_{l}(n)=\frac{n \log n_{l}}{\alpha_{l}} \log \left(a_{n}(q ; \omega, l)\right)$. Since $\max _{l} \sup _{n \in \mathbb{N}}\left|f_{l}(n+1)-f_{l}(n)\right|<\infty$ and

$$
\frac{1}{t} \sum_{l=1}^{d-1}\left(\alpha_{l} f_{l}\left(\left[\frac{t}{\log n_{l}}\right]\right)-f_{l}\left(\left[\alpha_{l} \frac{t}{\log n_{l}}\right]\right)\right)
$$




$$
=\log \left(\frac{\prod_{l=1}^{d-1} a_{k_{l}\left(e^{-t}\right)}(q ; \omega, l)^{h_{l}(t)}}{\prod_{l=1}^{d-1} a_{k_{l+1}\left(e^{-t}\right)}(q ; \omega, l)^{h_{l}\left(\alpha_{l} t\right)}}\right)
$$

with $h_{l}(t)=\frac{\left[\frac{t}{\log n_{l}}\right]}{\log n_{l}}$, Lemma 6.1.4 shows that

$$
\limsup _{t \rightarrow \infty} \frac{\prod_{l=1}^{d-1} a_{k_{l}\left(e^{-t}\right)}(q ; \omega, l)^{h_{l}(t)}}{\prod_{l=1}^{d-1} a_{k_{l+1}\left(e^{-t}\right)}(q ; \omega, l)^{h_{l}\left(\alpha_{l} t\right)}} \geq 1 .
$$

Let $\varepsilon>0$. Choose $t_{0}>0$ such that $1-\varepsilon \leq h_{l}(t), h_{l}\left(\alpha_{l} t\right) \leq 1+\varepsilon$ for $l=1, \ldots, d-1$ and $t>t_{0}$. Equations (6.1) and (6.4) now imply that

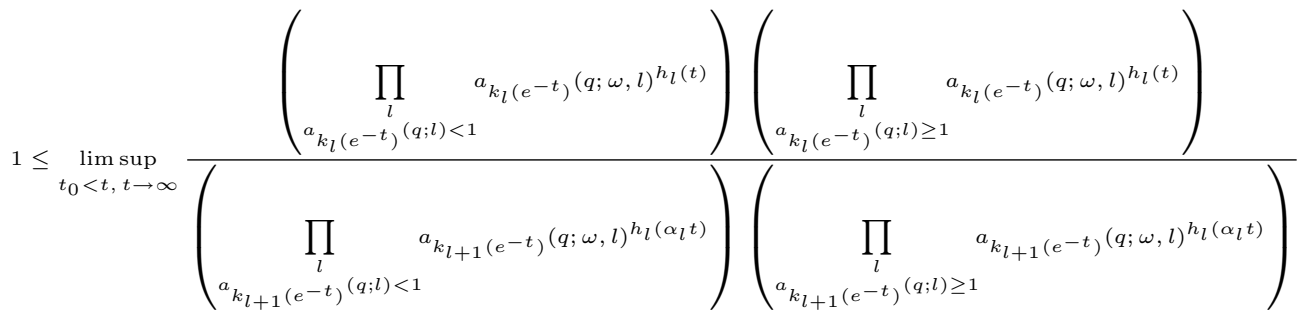

$$
\begin{aligned}
& \left(\prod_{l=1}^{d-1} a_{k_{l}\left(e^{-t}\right)}(q ; \omega, l)\right) \frac{\prod_{l} a_{k_{l}\left(e^{-t}\right)}(q ; \omega, l)^{\varepsilon}}{\prod_{l}^{a_{l}\left(e^{-t)}(q ; l) \geq 1\right.}} \\
& \leq \limsup _{t_{0}<t, t \rightarrow \infty} \frac{a_{k_{l}\left(e^{-t}\right)}(q ; l)<1}{\prod_{l} a_{k_{l+1}\left(e^{-t)}\right.}(q ; \omega, l)^{\varepsilon}} \\
& \left(\prod_{l=1}^{d-1} a_{k_{l+1}\left(e^{-t}\right)}(q ; \omega, l)\right) \frac{a_{k_{l+1}\left(e^{-t}\right)}(q ; l)<1}{\prod_{l} a_{k_{l+1}\left(e^{-t}\right)}(q ; \omega, l)^{\varepsilon}} \\
& a_{k_{l+1}\left(e^{-t}\right)}(q ; l) \geq 1 \\
& \leq\left(\frac{A(q)}{a(q)}\right)^{2 \varepsilon d} \limsup _{t \rightarrow \infty} \frac{\prod_{l=1}^{d-1} a_{k_{l}\left(e^{-t}\right)}(q ; \omega, l)}{\prod_{l=1}^{d-1} a_{k_{l+1}\left(e^{-t}\right)}(q ; \omega, l)}
\end{aligned}
$$

for all $\varepsilon>0$. Letting $\varepsilon \searrow 0$ yields the desired result.

Proposition 6.1.6. Let $q \in \mathbb{R}$ and $\omega \in \Sigma$. Then

$$
\limsup _{r \searrow 0} D_{r}(q ; \omega) \geq 1 .
$$

Proof. Write $\omega=\left(\mathbf{i}_{1}, \mathbf{i}_{2}, \ldots\right)$. Since

$$
\lim _{r \searrow 0} \frac{1}{\alpha_{1} \cdots \alpha_{l-1}} \frac{k_{l}(r)}{k_{1}(r)}=1 \quad \text { and } \quad \lim _{\searrow 0}\left(1-\frac{1}{\alpha_{l}} \frac{k_{l+1}(r)}{k_{l}(r)}\right)=0
$$


inequality (6.1) implies that $\prod_{\substack{l \\ \alpha_{l} \neq 1}}\left(a_{k_{l+1}(r)}(q ; \omega, l)^{1-\frac{1}{\alpha_{l}} \frac{k_{l+1}(r)}{k_{l}(r)}}\right)^{\frac{1}{\alpha_{1} \cdots \alpha_{l-1}} \frac{k_{l}(r)}{k_{1}(r)}} \rightarrow 1$ as $r \searrow 0$. We therefore infer from Lemma 6.1.3 that

$$
\limsup _{r \searrow 0} D_{r}(q ; \omega)=\limsup _{r \searrow 0} \prod_{\alpha_{l} \neq 1}\left(\frac{a_{k_{l}(r)}(q ; \omega, l)}{a_{k_{l+1}(r)}(q ; \omega, l)}\right)^{\frac{1}{\alpha_{1} \cdots \alpha_{l-1}} \frac{k_{l}(r)}{k_{1}(r)}} .
$$

Let $\varepsilon>0$. There exists $r_{0}>0$ such that $1-\varepsilon \leq \frac{1}{\alpha_{1} \cdots \alpha_{l-1}} \frac{k_{l}(r)}{k_{1}(r)} \leq 1+\varepsilon$ for $l=1, \ldots, d-1$ and $r<r_{0}$. Hence, using (6.5) and Lemma 6.1.5,

$$
\begin{aligned}
& \limsup _{r \searrow 0} D_{r}(q ; \omega) \geq \limsup _{r_{0}>r, r \searrow 0}\left(\prod_{\substack{l \\
\frac{a_{k_{l}(r)}(q ; \omega, l)}{a_{k_{l+1}(r)}(q ; \omega, l)}<1}}\left(\frac{a_{k_{l}(r)}(q ; \omega, l)}{a_{k_{l+1}(r)}(q ; \omega, l)}\right)^{1+\varepsilon}\right) \\
& \cdot\left(\prod_{\substack{l \\
\frac{a_{k_{l}(r)}(q ; \omega, l)}{a_{k_{l+1}(r)}(q ; \omega, l)} \geq 1}}\left(\frac{a_{k_{l}(r)}(q ; \omega, l)}{a_{k_{l+1}(r)}(q ; \omega, l)}\right)^{1-\varepsilon}\right)
\end{aligned}
$$

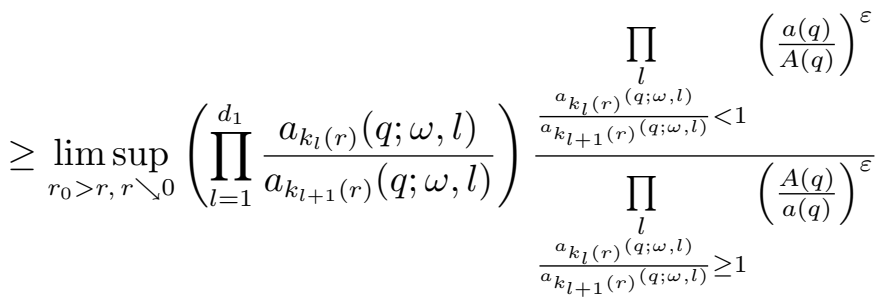

$$
\begin{aligned}
& \geq\left(\frac{a(q)}{A(q)}\right)^{2 \varepsilon d} \limsup _{r \searrow 0} \prod_{l=1}^{d-1} \frac{a_{k_{l}(r)}(q ; \omega, l)}{a_{k_{l+1}(r)}(q ; \omega, l)} \geq\left(\frac{a(q)}{A(q)}\right)^{2 \varepsilon d}
\end{aligned}
$$

for all $\varepsilon>0$. Letting $\varepsilon \searrow 0$ now yields the desired result.

The last two lemmas in this section give explicit expressions for $\alpha(q)=$ $-\beta^{\prime}(q)$ and $q \alpha(q)+\beta(q)$.

Lemma 6.1.7. For $q \in \mathbb{R}$,

$$
\alpha(q)=-\sum_{l=1}^{d} \frac{1}{\log n_{l}} \sum_{\left(i_{1}, \ldots, i_{l}\right) \in I_{l}} Q_{l}\left(q ; i_{1}, \ldots, i_{l}\right) \log p_{l}\left(i_{l} \mid i_{1}, \ldots, i_{l-1}\right)
$$


(recall that $\left.\alpha=-\beta^{\prime}\right)$.

Proof. The definitions of $\beta, \beta_{i_{1}}, \beta_{i_{1} i_{2}}, \ldots, \beta_{i_{1} \ldots i_{d}}$ imply that

$$
\begin{aligned}
& -\beta_{i_{1} \ldots i_{l-1}}^{\prime}(q) \\
& =-\frac{1}{\log n_{l}} \sum_{\substack{i_{l} \\
\left(i_{1}, \ldots, i_{l}\right) \in I_{l}}} p_{l}\left(i_{l} \mid i_{1}, \ldots, i_{l-1}\right)^{q} n_{l}^{\beta_{i_{1} \ldots i_{l}}(q)-\beta_{i_{1} \ldots i_{l-1}}(q)} \log p_{l}\left(i_{l} \mid i_{1} \ldots i_{l-1}\right) \\
& \quad-\sum_{\substack{i_{l} \\
\left(i_{1}, \ldots, i_{l}\right) \in I_{l}}} p_{l}\left(i_{l} \mid i_{1}, \ldots, i_{l-1}\right)^{q} n_{l}^{\beta_{i_{1} \ldots i_{l}}(q)-\beta_{i_{1} \ldots i_{l-1}}(q)} \beta_{i_{1} \ldots i_{l}}^{\prime}(q)
\end{aligned}
$$

for $l=1, \ldots, d$. It follows from repeated application of (6.6), Lemma 6.1.2 and the fact that $\beta_{i_{1} \ldots i_{d}}(q)=0$ for all $q$ that

$$
\begin{aligned}
- & \beta^{\prime}(q) \\
= & -\sum_{l=1}^{d} \frac{1}{\log n_{l}} \sum_{\substack{i_{1} \\
i_{1} \in I_{1}}} \sum_{\substack{i_{2} \\
\left(i_{1}, i_{2}\right) \in I_{2}}} \ldots \sum_{\substack{i_{l} \\
\left(i_{1}, \ldots, i_{l}\right) \in I_{l}}} q_{l}\left(i_{1}, \ldots, i_{l}\right)^{q} \\
& \cdot n_{l}^{\beta_{i_{1} \ldots i_{l}}(q)-\beta_{i_{1} \ldots i_{l-1}}(q)} \ldots n_{2}^{\beta_{i_{1} i_{2}}(q)-\beta_{i_{1}}(q)} n_{1}^{\beta_{i_{1}}(q)-\beta(q)} \log p_{d}\left(i_{l} \mid i_{1}, \ldots, i_{l-1}\right) \\
= & -\sum_{l=1}^{d} \frac{1}{\log n_{l}} \sum_{\substack{\left(i_{1}, \ldots, i_{l}\right) \in I_{l}\\
}} Q_{l}\left(q ; i_{1}, \ldots, i_{l}\right) \log p_{d}\left(i_{l} \mid i_{1}, \ldots, i_{l-1}\right) .
\end{aligned}
$$

Lemma 6.1.8. For $q \in \mathbb{R}$,

$$
q \alpha(q)+\beta(q)=-\sum_{l=1}^{d} \frac{1}{\log n_{l}} \sum_{\left(i_{1}, \ldots, i_{l}\right) \in I_{l}} Q_{l}\left(q ; i_{1}, \ldots, i_{l}\right) \log P_{l}\left(q ; i_{l} \mid i_{1}, \ldots, i_{l-1}\right)
$$

(recall that $\left.\alpha=-\beta^{\prime}\right)$.

Proof. For each $l=1, \ldots, d$ we get by applying Lemma 6.1.2,

$$
\begin{aligned}
& \log P_{l}\left(q ; i_{l} \mid i_{1}, \ldots, i_{l-1}\right) \\
& =\log \frac{Q_{l}\left(q ; i_{1}, \ldots, i_{l}\right)}{Q_{l}\left(q ; i_{1}, \ldots, i_{l-1}\right)} \\
& =\log \left(\left(\frac{q_{l}\left(i_{1}, \ldots, i_{l}\right)}{q_{l}\left(i_{1}, \ldots, i_{l-1}\right)}\right)^{q} n_{l}^{\beta_{i_{1} \ldots i_{l}}(q)-\beta_{i_{1} \ldots i_{l-1}}(q)}\right)
\end{aligned}
$$




$$
=q \log p_{l}\left(i_{l} \mid i_{1}, \ldots, i_{l-1}\right)+\left(\beta_{i_{1} \ldots i_{l}}(q)-\beta_{i_{1} \ldots i_{l-1}}(q)\right) \log n_{l} .
$$

Let $\Phi(q)$ denote the righthand side of Equation (6.7). It follows from (6.8) and the previous lemma that

$$
\begin{aligned}
& \Phi(q)=-q \sum_{l=1}^{d}\left(\frac{1}{\log n_{l}} \sum_{\left(i_{1}, \ldots, i_{l}\right) \in I_{l}} Q_{l}\left(q ; i_{1}, \ldots, i_{l}\right) \log p_{l}\left(i_{l} \mid i_{1}, \ldots, i_{l-1}\right)\right) \\
& -\sum_{l=1}^{d}\left(\frac{1}{\log n_{l}} \sum_{\left(i_{1}, \ldots, i_{l}\right) \in I_{l}} Q_{l}\left(q ; i_{1}, \ldots, i_{l}\right)\left(\beta_{i_{1} \ldots i_{l}}(q)-\beta_{i_{1} \ldots i_{l-1}}(q)\right) \log n_{l}\right) \\
& =q \alpha(q)-\sum_{l=1}^{d}\left(\sum_{\left(i_{1}, \ldots, i_{l}\right) \in I_{l}} Q_{l}\left(q ; i_{1}, \ldots, i_{l}\right)\left(\beta_{i_{1} \ldots i_{l}}(q)-\beta_{i_{1} \ldots i_{l-1}}(q)\right)\right) \\
& =q \alpha(q)+\sum_{i_{1} \in I_{1}} Q_{1}\left(q ; i_{1}\right) \beta(q)-\sum_{l=1}^{d-1}\left(\sum_{\left(i_{1}, \ldots, i_{l}\right) \in I_{l}} Q_{l}\left(q ; i_{1}, \ldots, i_{l}\right) \beta_{i_{1} \ldots i_{l}}(q)\right) \\
& +\sum_{l=1}^{d-1}\left(\sum_{\left(i_{1}, \ldots, i_{l}\right) \in I_{l}} Q_{l}\left(q ; i_{1}, \ldots, i_{l}\right) \beta_{i_{1} \ldots i_{l}}(q)\right. \\
& \text {. } \left.\quad \sum_{i_{l+1}} P_{l+1}\left(q ; i_{l+1} \mid i_{1}, \ldots, i_{l}\right)\right) \\
& \left(i_{1}, \ldots, i_{l+1}\right) \in I_{l+1} \\
& =q \alpha(q)+\beta(q)
\end{aligned}
$$

which completes the proof.

\subsection{Separation results.}

In this section we deduce some of the consequences of separartion condition (II).

Proposition 6.2.1. Let $\omega \in \Sigma$ and $n \in \mathbb{N}$.

i) If condition (II) is satisfied then $B\left(\pi(\omega), \frac{1}{2 n_{1}^{n}}\right) \cap K \subseteq Q\left(\omega, \frac{1}{n_{1}^{n}}\right)$.

ii) $Q\left(\omega, \frac{1}{n_{1}^{n}}\right) \subseteq B\left(\pi(\omega),\left(n_{1}+\cdots+n_{d}\right) \frac{1}{n_{1}^{n}}\right)$.

Proof. i) Write $\omega=\left(\mathbf{i}_{1}, \mathbf{i}_{2}, \ldots\right) \in \Sigma$ with $\mathbf{i}_{m}=\left(i_{m, 1}, \ldots, i_{m, d}\right)$. Let $\pi(\sigma) \in$ $B\left(\pi(\omega), \frac{1}{2 n_{1}^{n}}\right) \cap K$ with $\sigma=\left(\mathbf{j}_{1}, \mathbf{j}_{2}, \ldots\right) \in \Sigma$ and $\mathbf{j}_{m}=\left(j_{m, 1}, \ldots, j_{m, d}\right)$. We must now prove that $\pi(\sigma) \in Q\left(\omega, \frac{1}{n_{1}^{n}}\right)$. Since $k_{l}\left(\frac{1}{n_{1}^{n}}\right)=\left[\frac{n \log n_{1}}{\log n_{l}}\right]=$ $\left[\alpha_{1} \cdots \alpha_{l-1} n\right], \pi(\sigma) \in Q\left(\omega, \frac{1}{n_{1}^{n}}\right)$ if and only if

$$
i_{1, l}=j_{1, l}, \ldots, i_{\left[\alpha_{1} \cdots \alpha_{l-1} n\right], l}=j_{\left[\alpha_{1} \cdots \alpha_{l-1} n\right], l} \quad \text { for all } l=1, \ldots, d \text {. }
$$


Assume now, in order to get a contradiction, that (6.9) is not satisfied. We can then choose $l_{0} \in\{1, \ldots, d\}$ and $m_{0} \in\left\{1, \ldots,\left[\alpha_{1} \cdots \alpha_{l_{0}-1} n\right]-1\right\}$ such that

$$
\begin{gathered}
i_{m, 1}=j_{m, 1} \\
i_{m, 2}=j_{m, 2} \\
\quad \text { for } m=1, \ldots,\left[\alpha_{1} n\right] \\
\quad \vdots \\
i_{m, l_{0}-1}=j_{m, l_{0}-1} \quad \text { for } m=1, \ldots,\left[\alpha_{1} \cdots \alpha_{l_{0}-2} n\right] \\
i_{1, l_{0}}=j_{1, l_{0}}, \ldots, i_{m_{0}, l_{0}}=j_{m_{0}, l_{0}}, i_{m_{0}+1, l_{0}} \neq j_{m_{0}+1, l_{0}} .
\end{gathered}
$$

For $l=1, \ldots, d$ define projections $\Pi_{l}: \mathbb{R}^{d} \rightarrow \mathbb{R}$ by $\Pi_{l}\left(x_{1}, \ldots, x_{d}\right)=x_{l}$. It follows from (II) and (6.10) that $i_{m_{0}+1, l}=j_{m_{0}+1, l}$ for $l=1, \ldots, l_{0}-1$ and $\left|i_{m_{0}+1, l_{0}}-j_{m_{0}+1, l_{0}}\right|>1$, whence

$$
\operatorname{dist}\left(\Pi_{l_{0}} S_{\mathbf{i}_{m_{0}+1}}\left([0,1]^{d}\right), \Pi_{l_{0}} S_{\mathbf{j}_{m_{0}+1}}\left([0,1]^{d}\right)\right) \geq \frac{1}{n_{l_{0}}} .
$$

Since $\pi(\omega) \in S_{\mathbf{i}_{1}} \circ \cdots \circ S_{\mathbf{i}_{m_{0}+1}}\left([0,1]^{d}\right), \pi(\sigma) \in S_{\mathbf{j}_{1}} \circ \cdots \circ S_{\mathbf{j}_{m_{0}+1}}\left([0,1]^{d}\right)$ and $i_{m, l_{0}}=j_{m, l_{0}}$ for $m=1, \ldots, m_{0},(6.11)$ implies that

$$
\begin{aligned}
& \left|\Pi_{l_{0}} \pi(\omega)-\Pi_{l_{0}} \pi(\sigma)\right| \\
& \geq \operatorname{dist}\left(\Pi_{l_{0}} S_{\mathbf{i}_{1}} \circ \cdots \circ S_{\mathbf{i}_{m_{0}+1}}\left([0,1]^{d}\right), \Pi_{l_{0}} S_{\mathbf{j}_{1}} \circ \cdots \circ S_{\mathbf{j}_{m_{0}+1}}\left([0,1]^{d}\right)\right) \\
& \geq \frac{1}{n_{l_{0}}^{m_{0}+1}} \geq \frac{1}{n_{l_{0}}^{\left[\alpha_{1} \cdots \alpha_{l_{0}-1} n\right]}} .
\end{aligned}
$$

However, we clearly have $\left|\Pi_{l_{0}} \pi(\omega)-\Pi_{l_{0}} \pi(\sigma)\right| \leq|\pi(\omega)-\pi(\sigma)|<\frac{1}{n_{1}^{n}} \leq$

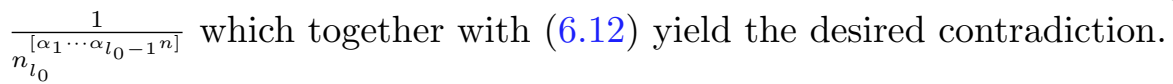

ii) This inclusion follows immediately from the definition of $Q\left(\omega, \frac{1}{n_{1}^{n}}\right)$ and the fact that $k_{l}\left(\frac{1}{n_{1}^{n}}\right)=\left[\alpha_{1} \cdots \alpha_{l-1} n\right]$ and $\frac{1}{n_{l}^{\left[\alpha_{1} \cdots \alpha_{l-1}\right]}} \leq \frac{n_{l}}{n_{1}^{n}}$.

Theorem 6.2.2. Assume that condition (II) holds. Then

$$
\Delta_{\mu}(\alpha)=\Delta_{\mu}^{\mathbf{c}}(\alpha)
$$

Proof. Proposition 6.2.1 clearly implies that

$$
\lim _{r \searrow 0} \frac{\log \mu Q(\omega, r)}{\log r}=\alpha \quad \Leftrightarrow \quad \lim _{r \searrow 0} \frac{\log \mu B(\pi(\omega), r)}{\log r}=\alpha
$$


for all $\omega \in \Sigma$ and $\alpha \geq 0$ which yields the desired result.

Lemma 6.2.3. Let $c>1$. Then

$$
\chi_{c}:=\sup _{\omega \in \Sigma} \sup _{r>0} \frac{\mu Q(\omega, c r)}{\mu Q(\omega, r)}<\infty .
$$

Proof. Let $\left.p_{\min }=\min _{l=1, \ldots, d} \min _{\left(i_{1}, \ldots, i_{l}\right) \in I_{l}} p_{l}\left(i_{l} \mid i_{1}, \ldots, i_{l-1}\right) \in\right] 0,1[$. Now fix $r>0$ and $\omega \in \Sigma$. Since $0 \leq k_{l}(r)-k_{l}(c r) \leq \frac{\log c}{\log n_{l}}+1$, (6.2) implies that

$$
\begin{aligned}
\frac{\mu Q(\omega, c r)}{\mu Q(\omega, r)} & =\frac{\prod_{l=1}^{d} \prod_{j=0}^{k_{l}(c r)-1} p_{l}\left(T^{j} \omega\right)}{\prod_{l=1}^{d} \prod_{j=0}^{k_{l}(r)-1} p_{l}\left(T^{j} \omega\right)} \leq \prod_{l=1}^{d} \prod_{j=k_{l}(c r)}^{k_{l}(r)-1} p_{\min }^{-1} \\
& =\prod_{l=1}^{d} p_{\min }^{-\left(k_{l}(r)-k_{l}(c r)\right)} \leq \prod_{l=1}^{d} p_{\min }^{-\left(\frac{\log c}{\log n_{l}}+1\right)}
\end{aligned}
$$

which completes the proof.

\subsection{Auxiliary density results.}

We first collect some well-known density results.

Theorem 6.3.1. Let $\nu$ be a regular Borel measure in $\mathbb{R}^{d}, E \subseteq \mathbb{R}^{d}, t>0$ and $0<\lambda<\infty$. If

$$
\limsup _{r \searrow 0} \frac{\nu B(x, r)}{(2 r)^{t}} \geq \lambda \quad \text { for } x \in E,
$$

then

$$
\mathcal{H}^{t}(E) \leq \frac{1}{\lambda} \nu(E)
$$

Proof. See [Mat, Theorem 6.9].

Theorem 6.3.2. For each $x \in \mathbb{R}$ let $x=\sum_{i=1}^{\infty} \frac{\varepsilon_{i, l}(x)}{n_{l}^{i}}$ denote the unique nonterminating $n_{l}$-adic expansion of $x$. For $x=\left(x_{1}, \ldots, x_{d}\right) \in \mathbb{R}^{d}$ write $\varepsilon_{i}(x)=$ $\left(\varepsilon_{i, l}\left(x_{l}\right)\right)_{l=1, \ldots, d}$ and put $\omega(x)=\left(\varepsilon_{1}(x), \varepsilon_{2}(x), \ldots\right) \in\left(\prod_{l=1}^{d}\left\{0, \ldots, n_{l}-1\right\}\right)^{\mathbb{N}}$. If $\nu \in \mathcal{P}\left(\mathbb{R}^{d}\right), \alpha \geq 0$ and

$$
\lim _{r \searrow 0} \frac{\log \nu Q(\omega(x), r)}{\log r}=\alpha \quad \text { for } \nu \text {-a.a. } x,
$$

then

$$
\operatorname{dim} \nu=\operatorname{Dim} \nu=\alpha
$$


Proof. These results are proved in e.g. [Cu2], [Ha, Proposition 1], [Mat, Theorem 6.9 and Theorem 6.11] or [Yo] using balls in place of approximate cubes, but the proofs transfer since the ratio of the sidelengths of an approximate cube is at most $n_{d}$ and we can thus in the definitions of the Hausdorff and packing measures restrict our attention to covers by approximate cubes.

Next we prove some small technical density lemmas.

Lemma 6.3.3. Let $q \in \mathbb{R}$. Then

i) $\lim _{r \searrow 0} \frac{\log \mu(Q(\omega, r))}{\log r}=-\sum_{l=1}^{d} \frac{1}{\log n_{l}} \sum_{\left(i_{1}, \ldots, i_{l}\right) \in I_{l}} q_{l}\left(i_{1}, \ldots, i_{l}\right) \log p_{l}\left(i_{l} \mid i_{1}\right.$, $\left.\ldots, i_{l-1}\right)$ for $\tilde{\mu}-a . a . \omega \in \Sigma$.

ii) $\lim _{r \searrow 0} \frac{\log \mu(Q(\omega, r))}{\log r}=\alpha(q)$ for $\tilde{\mu}^{q}-a . a . \omega \in \Sigma$.

iii) $\lim _{r \searrow 0} \frac{\log \mu^{q}(Q(\omega, r))}{\log r}=q \alpha(q)+\beta(q)$ for $\tilde{\mu}^{q}-a . a . \omega \in \Sigma$.

Proof. i) It follows from (6.2) that

$$
\frac{\log \mu(Q(\omega, r))}{\log r}=-\sum_{l=1}^{d} \frac{k_{l}(r)}{\log \frac{1}{r}} \frac{1}{k_{l}(r)} \sum_{j=0}^{k_{l}(r)-1} \log p_{l}\left(T^{j} \omega\right)
$$

for $\omega \in \Sigma$ and $r>0$. The ergodic theorem implies that

$$
\begin{aligned}
\frac{1}{k} \sum_{j=0}^{k-1} \log p_{l}\left(T^{j} \omega\right) & \rightarrow \sum_{\mathbf{i}=\left(i_{1}, \ldots, i_{d}\right) \in I} p_{\mathbf{i}} \log p_{l}\left(i_{l} \mid i_{1}, \ldots, i_{l-1}\right) \\
& =\sum_{\left(i_{1}, \ldots, i_{l}\right) \in I_{l}}\left(\sum_{\substack{\mathbf{j}=\left(j_{1}, \ldots, j_{d}\right) \in I \\
j_{1}=i_{1}, \ldots, j_{l}=i_{l}}} p_{\mathbf{j}}\right) \log p_{l}\left(i_{l} \mid i_{1}, \ldots, i_{l-1}\right) \\
& =\sum_{\left(i_{1}, \ldots, i_{l}\right) \in I_{l}} q_{l}\left(i_{1}, \ldots, i_{l}\right) \log p_{l}\left(i_{l} \mid i_{1}, \ldots, i_{l-1}\right)
\end{aligned}
$$

$$
\text { as } k \rightarrow \infty \text { for } \tilde{\mu} \text {-a.a. } \omega \in \Sigma \text {. }
$$

Since $\frac{k_{l}(r)}{\log \frac{1}{r}} \rightarrow \frac{1}{\log n_{l}}$ as $r \searrow 0,(6.13)$ and (6.14) imply that

$$
\lim _{r \searrow 0} \frac{\log \mu(Q(\omega, r))}{\log r}=-\sum_{l=1}^{d} \frac{1}{\log n_{l}} \sum_{\left(i_{1}, \ldots, i_{l}\right) \in I_{l}} q_{l}\left(i_{1}, \ldots, i_{l}\right) \log p_{l}\left(i_{l} \mid i_{1}, \ldots, i_{l-1}\right)
$$

for $\tilde{\mu}$-a.a. $\omega \in \Sigma$.

ii) It follows from (6.3) and an argument similar to the proof of (6.15) that

$$
\lim _{r \searrow 0} \frac{\log \mu(Q(\omega, r))}{\log r}
$$




$$
\begin{gathered}
=-\sum_{l=1}^{d} \frac{1}{\log n_{l}} \sum_{\substack{\left(i_{1}, \ldots, i_{l}\right) \in I_{l} \\
\text { for } \tilde{\mu}^{q} \text {-a.a. } \omega \in \Sigma,}} Q_{l}\left(q ; i_{1}, \ldots, i_{l}\right) \log p_{l}\left(i_{l} \mid i_{1}, \ldots, i_{l-1}\right) \\
\end{gathered}
$$

and Lemma 6.1.7 therefore shows that $\lim _{r \backslash 0} \frac{\log \mu(Q(\omega, r))}{\log r}=\alpha(q)$ for $\tilde{\mu}^{q}$-a.a. $\omega \in \Sigma$.

iii) It follows from (6.3) and an argument similar to the proof of (6.15) that

$$
\begin{aligned}
& \lim _{r \searrow 0} \frac{\log \mu^{q}(Q(\omega, r))}{\log r} \\
& =-\sum_{l=1}^{d} \frac{1}{\log n_{l}} \sum_{\left(\begin{array}{c}
\left.i_{1}, \ldots, i_{l}\right) \in I_{l} \\
\text { for } \tilde{\mu}^{q} \text {-a.a. } \omega \in \Sigma,
\end{array}\right.} Q_{l}\left(q ; i_{1}, \ldots, i_{l}\right) \log P_{l}\left(q ; i_{l} \mid i_{1}, \ldots, i_{l-1}\right) \\
& \quad
\end{aligned}
$$

and Lemma 6.1.8 therefore shows that $\lim _{r \backslash 0} \frac{\log \mu^{q}(Q(\omega, r))}{\log r}=q \alpha(q)+\beta(q)$ for $\tilde{\mu}^{q}$-a.a. $\omega \in \Sigma$.

Lemma 6.3.4. Let $q \in \mathbb{R}$. Then

$$
D_{r}(q ; \omega) \rightarrow 1 \quad \text { as } r \searrow 0 \text { for } \tilde{\mu}^{q} \text {-a.a. } \omega \in \Sigma .
$$

Proof. Lemma 6.3.3 together with the fact that $\lim _{r \backslash 0} \frac{\log r}{k_{1}(r)}=-\log n_{1}$ imply that

$$
\begin{aligned}
\log D_{r}(q ; \omega) & =\frac{\log r}{k_{1}(r)} \frac{\log \mu^{q}(Q(\omega, r))}{\log r}-q \frac{\log r}{k_{1}(r)} \frac{\log \mu(Q(\omega, r))}{\log r}+\beta(q) \log n_{1} \\
& \rightarrow-\log n_{1}(q \alpha(q)+\beta(q))-q\left(-\log n_{1}\right) \alpha(q)+\beta(q) \log n_{1} \\
& =0 \quad \text { as } r \searrow 0 \text { for } \tilde{\mu}^{q} \text {-a.a. } \omega \in \Sigma .
\end{aligned}
$$

6.4. The multifractal Hausdorff dimension function $b_{\mu}$, and the Hausdorff spectrum.

Theorem 6.4.1.

1) If Case $1^{\prime}$ is satisfied then the following statement hold.

i) $f_{\mu}^{\mathrm{c}}(s)=\beta^{*}(s)=s$.

2) If Case $2^{\prime}$ is satisfied then the following statement hold.

i) $f_{\mu}^{\mathbf{c}}(\alpha)=\beta^{*}(\alpha)$ for $\left.\alpha \in\right] \underline{a}, \bar{a}[$.

Proof. Case 1': 
The proof of the statement in Case $1^{\prime}$ is very similar to the proof in Case $2^{\prime}$ and is therefore omitted.

Case 2':

Proof of: $f_{\mu}^{\mathbf{c}}(\alpha) \leq \beta^{*}(\alpha)$. We must prove that $f_{\mu}^{\mathbf{c}}(\alpha) \leq q \alpha+\beta(q)$ for all $q \in \mathbb{R}$. Now fix $q \in \mathbb{R}$. Since, by Proposition 6.1.6,

$$
\limsup _{r \searrow 0}\left(\frac{\mu^{q}(Q(\omega, r))}{\mu(Q(\omega, r))^{q}\left(\frac{1}{n_{1}^{k_{1}(r)}}\right)^{\beta(q)}}\right)^{\frac{1}{k_{1}(r)}}=\limsup _{r \searrow 0} D_{r}(q ; \omega) \geq 1 \text { for all } \omega \in \Sigma,
$$

we deduce that

$$
\begin{aligned}
\liminf _{r \searrow 0} \frac{\log \mu^{q}(Q(\omega, r))}{\log r} & \leq q \lim _{r \searrow 0}\left(\frac{\log \mu(Q(\omega, r))}{\log r}\right)+\beta(q) \\
& \leq q \alpha_{\mu}^{\mathbf{c}}(\omega)+\beta(q) \\
& =q \alpha+\beta(q) \quad \text { for } \omega \in \pi^{-1}\left(\Delta_{\mu}^{\mathbf{c}}(\alpha)\right) .
\end{aligned}
$$

Hence

$$
\limsup _{r \searrow 0} \frac{\mu^{q}(Q(\omega, r))}{r^{q \alpha+\beta(q)+\varepsilon}}=\infty \quad \text { for all } \omega \in \pi^{-1}\left(\Delta_{\mu}^{\mathbf{c}}(\alpha)\right) \text { and } \varepsilon>0 .
$$

It thus follows from Theorem 6.3.1 that $\mathcal{H}^{q \alpha+\beta(q)+\varepsilon}\left(\Delta_{\mu}^{\mathbf{c}}(\alpha)\right) \leq 1$ for all $\varepsilon>0$, whence $f_{\mu}^{\mathbf{c}}(\alpha)=\operatorname{dim} \Delta_{\mu}^{\mathbf{c}}(\alpha) \leq q \alpha+\beta(q)$.

Proof of: $f_{\mu}^{\mathbf{c}}(\alpha) \geq \beta^{*}(\alpha)$. Let $\left.\alpha \in\right] \underline{a}, \bar{a}[$. By Proposition 3.2.1 and Theorem 3.2.2 there exists a (unique) $q \in \mathbb{R}$ such that $\alpha=\alpha(q)$. It follows from Theorem 3.4.1.v), Lemma 6.3.3 and Theorem 6.3.2 that $\operatorname{dim} \mu^{q}=$ $q \alpha(q)+\beta(q)=\beta^{*}(\alpha(q))=\beta^{*}(\alpha)$ and $\mu^{q}\left(\Delta_{\mu}^{\mathbf{c}}(\alpha)\right)=\mu^{q}\left(\Delta_{\mu}^{\mathbf{c}}(\alpha(q))\right)=1$. Hence $f_{\mu}^{\mathbf{c}}(\alpha)=\operatorname{dim} \Delta_{\mu}^{\mathbf{c}}(\alpha) \geq \operatorname{dim} \mu^{q}=\beta^{*}(\alpha)$.

Theorem 6.4.2. Assume that condition (II) holds. Let $q \in \mathbb{R}$ and $\varepsilon>0$ Then there exists a constant $c_{q, \varepsilon}>0$ such that

$$
c_{q, \varepsilon} \mu^{q} \leq \mathcal{H}_{\mu}^{q, \beta(q)-\varepsilon}\llcorner K .
$$

Proof. It follows from Proposition 6.2.1 and Lemma 6.2.3 that there exists a constant $c>0$ such that

$$
\begin{aligned}
& \left(\frac{\mu B(\pi(\omega), r)}{\mu Q\left(\omega, \frac{1}{n_{1}^{m}}\right)}\right)^{q} \geq c, \quad(2 r)^{\beta(q)-\varepsilon} \geq c\left(\frac{1}{n_{1}^{m}}\right)^{\beta(q)-\varepsilon} \\
& \quad \text { for } \omega \in \Sigma, m \in \mathbb{N} \text { and } \frac{1}{2 n_{1}^{m+1}} \leq r<\frac{1}{2 n_{1}^{m}}
\end{aligned}
$$


Let $c_{q, \varepsilon}=c^{2}$.

Fix a Borel subset $B$ of $K$. For $n \in \mathbb{N}$ write

$$
\Sigma_{n}=\left\{\omega \mid \omega \in \pi^{-1}(B), D_{n_{1}^{-m}}(q ; \omega)<n_{1}^{\varepsilon} \quad \text { for } m \geq n\right\}, F_{n}=\pi\left(\Sigma_{n}\right) \subseteq B .
$$

Now fix $n \in \mathbb{N}$. Let $\left(B\left(x_{i}, r_{i}\right)\right)_{i \in \mathbb{N}}$ be a centered $\frac{1}{2} n_{1}^{-(n+1)}$-covering of $F_{n}$. For each $i \in \mathbb{N}$ choose $\omega_{i} \in \Sigma$ such that $\pi\left(\omega_{i}\right)=x_{i}$, and let $m_{i}$ be the unique integer satisfying

$$
\frac{1}{2 n_{1}^{m_{i}+1}} \leq r_{i}<\frac{1}{2 n_{1}^{m_{i}}} \text {. }
$$

Next observe that $\frac{1}{2 n_{1}^{m_{i}+1}} \leq r_{i} \leq \frac{1}{2 n_{1}^{n+1}}$ for all $i$. Hence, $m_{i} \geq n$ for all $i$, whence $\omega_{i} \in \Sigma_{n} \subseteq \Sigma_{m_{i}}$, and so

$$
D_{n_{1}^{-m_{i}}}\left(q ; \omega_{i}\right)<n_{1}^{\varepsilon} \quad \text { for all } i .
$$

It follows from (6.16) and (6.17) that

$$
\begin{aligned}
& \sum_{i} \mu\left(B\left(x_{i}, r_{i}\right)\right)^{q}\left(2 r_{i}\right)^{\beta(q)-\varepsilon} \\
& =\sum_{i}\left(\frac{\mu B\left(\pi\left(\omega_{i}\right), r_{i}\right)}{\mu Q\left(\omega_{i}, \frac{1}{n_{1}^{m_{i}}}\right)}\right)^{q} \frac{\mu Q\left(\omega_{i}, \frac{1}{n_{1}^{m_{i}}}\right)^{q}\left(2 r_{i}\right)^{\beta(q)-\varepsilon}}{\mu^{q}\left(Q\left(\omega_{i}, \frac{1}{n_{1}^{m_{i}}}\right)\right)} \mu^{q}\left(Q\left(\omega_{i}, \frac{1}{n_{1}^{m_{i}}}\right)\right) \\
& \geq c^{2} \sum_{i} \frac{1}{D_{n_{1}^{m_{i}}}\left(q ; \omega_{i}\right)^{m_{i}}} \mu^{q}\left(Q\left(\omega_{i}, \frac{1}{n_{1}^{m_{i}}}\right)\right) n_{1}^{m_{i} \varepsilon} \\
& \geq c_{q, \varepsilon} \sum_{i} \frac{1}{n_{1}^{m_{i} \varepsilon}} \mu^{q}\left(Q\left(\omega_{i}, \frac{1}{n_{1}^{m_{i}}}\right)\right) n_{1}^{m_{i} \varepsilon} \\
& \geq c_{q, \varepsilon} \mu^{q}\left(\bigcup_{i} B\left(x_{i}, r_{i}\right)\right) \geq c_{q, \varepsilon} \mu^{q}\left(F_{n}\right) .
\end{aligned}
$$

Hence

$$
\mathcal{H}_{\mu}^{q, \beta(q)-\varepsilon}(B) \geq \mathcal{H}_{\mu}^{q, \beta(q)-\varepsilon}\left(F_{n}\right) \geq \overline{\mathcal{H}}_{\mu, \frac{1}{2} n_{1}^{-(n+1)}}^{q, \beta(q)-\varepsilon}\left(F_{n}\right) \geq c_{q, \varepsilon} \mu^{q}\left(F_{n}\right),
$$

whence, since $F_{n} \nearrow \cup_{m} F_{m}$,

$$
\mathcal{H}_{\mu}^{q, \beta(q)-\varepsilon}(B) \geq c_{q, \varepsilon} \mu^{q}\left(\bigcup_{n} F_{n}\right) .
$$

Finally, since $\lim _{r \searrow 0} D_{r}(q ; \omega)=1$ for $\tilde{\mu}^{q}$-a.a. $\omega \in \Sigma, \mu^{q}\left(\cup_{n} F_{n}\right)=\mu^{q}(B)$, and so

$$
\mathcal{H}_{\mu}^{q, \beta(q)-\varepsilon}(B) \geq c_{q, \varepsilon} \mu^{q}\left(\bigcup_{n} F_{n}\right)=c_{q, \varepsilon} \mu^{q}(B) .
$$


Theorem 6.4.3. Assume that condition (II) holds. Then

$$
b_{\mu}=\beta \text {. }
$$

Proof of: $b_{\mu} \leq \beta$. It follows from Proposition 6.2.1 and Lemma 6.2.3 that there exists a constant $c>0$ such that

$$
\left(\frac{\mu B\left(\pi(\omega),\left(n_{1}+\cdots+n_{d}\right) \frac{1}{n_{1}^{m}}\right)}{\mu Q\left(\omega, \frac{1}{n_{1}^{m}}\right)}\right)^{q} \leq c \quad \text { for all } \omega \in \Sigma \text { and } m \in \mathbb{N} .
$$

Let $q \in \mathbb{R}$. Let $F \subseteq K$ and $\varepsilon>0$. For $r>0$ write

$$
\mathcal{Q}_{r}=\left\{Q(\omega, r) \mid \omega \in \pi^{-1}(F), D_{r}(q ; \omega)>n_{1}^{-\varepsilon}\right\} .
$$

Since $\lim \sup _{r \searrow 0} D_{r}(q ; \omega) \geq 1$ for all $\omega \in \Sigma$,

$$
F \subseteq \bigcup_{m \geq n}\left(\bigcup_{Q \in \mathcal{Q}_{n_{1}^{-m}}} Q\right) \quad \text { for all } n \in \mathbb{N}
$$

For each $m \in \mathbb{N}$ choose $\left(\omega_{m, i}\right)_{i \in \mathbb{N}} \subseteq \Sigma$ such that $\mathcal{Q}_{n_{1}^{-m}}=\left\{Q\left(\omega_{m, i}, n_{1}^{-m}\right) \mid\right.$ $i \in \mathbb{N}\}$. It follows from (6.19) and Proposition 6.2.1 that

$$
F \subseteq \bigcup_{m \geq n}\left(\bigcup_{i \in \mathbb{N}} B\left(\pi\left(\omega_{m, i}\right),\left(n_{1}+\cdots+n_{d}\right) \frac{1}{n_{1}^{m}}\right)\right) \quad \text { for all } n \in \mathbb{N} .
$$

Write $\delta_{m}=\left(n_{1}+\cdots+n_{d}\right) \frac{1}{n_{1}^{m}}$ for $m \in \mathbb{N}$. It follows from (6.18) and (6.20) that for each $n \in \mathbb{N}$,

$$
\begin{aligned}
& \overline{\mathcal{H}}_{\mu, \delta_{n}}^{q, \beta(q)+2 \varepsilon}(F) \\
& \leq \sum_{m \geq n} \sum_{i \in \mathbb{N}} \mu\left(B\left(\pi\left(\omega_{m, i}\right), \delta_{m}\right)\right)^{q}\left(2 \delta_{m}\right)^{\beta(q)+2 \varepsilon} \\
& \leq\left(2\left(n_{1}+\cdots+n_{d}\right)\right)^{\beta(q)+2 \varepsilon} \\
& \quad \cdot \sum_{m \geq n} \sum_{i \in \mathbb{N}}\left(\frac{\mu\left(B\left(\pi\left(\omega_{m, i}\right),\left(n_{1}+\cdots+n_{d}\right) \frac{1}{n_{1}^{m}}\right)\right)}{\mu\left(Q\left(\omega_{m, i}, \frac{1}{n_{1}^{m}}\right)\right)}\right)^{q} \\
& \quad \cdot \frac{\mu\left(Q\left(\omega_{m, i}, \frac{1}{n_{1}^{m}}\right)\right)^{q}\left(\frac{1}{n_{1}^{m}}\right)^{\beta(q)}}{\mu^{q}\left(Q\left(\omega_{m, i}, \frac{1}{n_{1}^{m}}\right)\right)} \mu^{q}\left(Q\left(\omega_{m, i}, \frac{1}{n_{1}^{m}}\right)\right)\left(\frac{1}{n_{1}^{m}}\right)^{2 \varepsilon}
\end{aligned}
$$




$$
\begin{aligned}
\leq & \left(2\left(n_{1}+\cdots+n_{d}\right)\right)^{\beta(q)+2 \varepsilon} c \\
& \cdot \sum_{m \geq n} \sum_{i \in \mathbb{N}} \frac{1}{D_{n_{1}^{-m}}\left(q ; \omega_{m, i}\right)^{k_{1}\left(\frac{1}{n_{1}^{m}}\right)}} \mu^{q}\left(Q\left(\omega_{m, i}, \frac{1}{n_{1}^{m}}\right)\right)\left(\frac{1}{n_{1}^{m}}\right)^{2 \varepsilon} \\
\leq & \left(2\left(n_{1}+\cdots+n_{d}\right)\right)^{\beta(q)+2 \varepsilon} c \\
& \cdot \sum_{m \geq n}\left(\frac{1}{n_{1}^{\varepsilon}}\right)^{m}\left(\sum_{\left(i_{1}, \ldots, i_{d}\right) \in \prod_{l=1}^{d}\left\{0, \ldots, n_{l}-1\right\}} 1\right) \\
= & \left(2\left(n_{1}+\cdots+n_{d}\right)\right)^{\beta(q)+2 \varepsilon} c n_{1} \cdots n_{d} \sum_{m \geq n}\left(\frac{1}{n_{1}^{\varepsilon}}\right)^{m} .
\end{aligned}
$$

Letting $n \rightarrow \infty$ thus yields $\overline{\mathcal{H}}_{\mu}^{q, \beta(q)+2 \varepsilon}(F)=0$ for all $F \subseteq K=\operatorname{supp} \mu$. Hence $\mathcal{H}_{\mu}^{q, \beta(q)+2 \varepsilon}(\operatorname{supp} \mu)=\sup _{F \subseteq \operatorname{supp} \mu} \overline{\mathcal{H}}_{\mu}^{q, \beta(q)+2 \varepsilon}(F)=0$, and so $b_{\mu}(q) \leq \beta(q)+2 \varepsilon$ for all $\varepsilon>0$.

Proof of: $b_{\mu} \geq \beta$. Let $q \in \mathbb{R}$ and $\varepsilon>0$. Theorem 6.4 .2 implies that $\mathcal{H}_{\mu}^{q, \beta(q)-\varepsilon}(\operatorname{supp} \mu) \geq c_{q, \varepsilon} \mu^{q}(\operatorname{supp} \mu)=c_{q, \varepsilon}>0$, whence $b_{\mu}(q)=\operatorname{dim}_{\mu}^{q}(\operatorname{supp} \mu)$ $\geq \beta(q)-\varepsilon$ for all $\varepsilon>0$.

Proof of Theorem 4.1.1.1) and Theorem 4.1.1.2). Follows from Theorem 6.4.1.

Proof of Corollary 4.1.2.i). Clearly $\operatorname{dim} K \geq \operatorname{dim} \Delta_{\mu}^{\mathbf{c}}(\alpha(0))=f_{\mu}^{\mathbf{c}}(\alpha(0))=$ $\beta^{*}(\alpha(0))=\beta(0)$, where we have used Theorem 6.4.1 and Theorem 3.4.1.v). Since, by Proposition 6.1.6,

$$
\limsup _{r \searrow 0}\left(\frac{\mu^{0} Q(\omega, r)}{\left(\frac{1}{n_{1}^{k_{1}(r)}}\right)^{\beta(0)}}\right)^{\frac{1}{k_{1}(r)}}=\limsup _{r \searrow 0} D_{r}(0 ; \omega) \geq 1 \quad \text { for all } \omega \in \Sigma,
$$

we deduce that $\liminf _{r \searrow 0} \frac{\log \mu^{0} Q(\omega, r)}{\log r} \leq \beta(0)$ for all $\omega \in \Sigma$. Hence $\lim \sup _{r \searrow 0} \frac{\mu^{0} Q(\omega, r)}{r^{\beta(0)+\varepsilon}}=\infty$ for all $\omega \in \Sigma$ and $\varepsilon>0$. It therefore follows from Theorem 6.3.1 that $\mathcal{H}^{\beta(0)+\varepsilon}(K) \leq 1$ for all $\varepsilon>0$, and so $\operatorname{dim} K \leq$ $\beta(0)$.

Proof of Theorem 4.1.3.i). Follows from Theorem 6.4.3.

Proof of Theorem 4.1.4. 
Case $1^{\prime}$ :

The proofs of the statements in Case $1^{\prime}$ are very similar to the proofs in Case $2^{\prime}$ and are therefore omitted.

Case 2':

i) It follows from Theorem 6.2.2 that $f_{\mu}(\alpha)=\operatorname{dim} \Delta_{\mu}(\alpha)=\operatorname{dim} \Delta_{\mu}^{\mathbf{c}}(\alpha)=$ $f_{\mu}^{\mathbf{c}}(\alpha)$ and from Theorem 6.4.1 that $f_{\mu}^{\mathbf{c}}(\alpha)=\beta^{*}(\alpha)$ for $\left.\alpha \in\right] \underline{a}, \bar{a}[$.

ii) It follows from Theorem 6.4.3 that $b_{\mu}=\beta$, and Proposition 3.2.1 therefore implies that

$$
\underline{a}_{\mu}=\sup _{0<q}-\frac{b_{\mu}(q)}{q}=\sup _{0<q}-\frac{\beta(q)}{q}=\underline{a}, \quad \bar{a}_{\mu}=\inf _{q<0}-\frac{b_{\mu}(q)}{q}=\inf _{q<0}-\frac{\beta(q)}{q}=\bar{a} .
$$

Theorem 2.1.3 now shows that $\Delta_{\mu}(\alpha)=\varnothing$ for $\alpha \in \mathbb{R}_{+} \backslash[\underline{a}, \bar{a}]$.

\subsection{The multifractal packing dimension function $B_{\mu}$.}

In this section we compute the multifractal packing dimension function $B_{\mu}$. We begin with some small lemmas.

Lemma 6.5.1. Let $\nu \in \mathcal{P}_{F}\left(\mathbb{R}^{d}\right)$ and $q, t \in \mathbb{R}$. Then

i) There exists a number $c>0$ such that $\overline{\mathcal{P}}_{\nu}^{q, t}(E) \leq \overline{\mathcal{P}}_{\nu}^{q, t}(\bar{E}) \leq c \overline{\mathcal{P}}_{\nu}^{q, t}(E)$ for all $E \subseteq \operatorname{supp} \nu$.

ii) $\Delta_{\nu}^{q}(E)=\Delta_{\nu}^{q}(\bar{E})$ for all $E \subseteq \operatorname{supp} \nu$.

Proof. i) Since $\nu \in \mathcal{P}_{F}\left(\mathbb{R}^{d}\right)$ there exists an $r_{0}>0$ and a number $c_{0}>0$ such that

$$
\left(\frac{\nu B(x, r)}{\nu B\left(y, \frac{1}{2} r\right)}\right)^{q} \leq c_{0} \quad \text { for } 0<r<r_{0} \text { and } x, y \in \operatorname{supp} \nu \text { with } y \in B\left(x, \frac{1}{2} r\right) .
$$

Let $E \subseteq \operatorname{supp} \nu, 0<\delta<r_{0}$ and $\left(B\left(x_{i}, r_{i}\right)\right)_{i \in \mathbb{N}}$ be a centered $\delta$-packing of $\bar{E}$. Choose $y_{i} \in B\left(x_{i}, \frac{1}{2} r_{i}\right) \cap E$ for each $i$. Since $B\left(y_{i}, \frac{1}{2} r_{i}\right) \subseteq B\left(x_{i}, r_{i}\right)$, $\left(B\left(y_{i}, \frac{1}{2} r_{i}\right)\right)_{i}$ is a centered $\frac{1}{2} \delta$-packing of $E$. Hence,

$$
\sum_{i} \nu\left(B\left(x_{i}, r_{i}\right)\right)^{q}\left(2 r_{i}\right)^{t} \leq 2^{t} c_{0} \sum_{i} \nu\left(B\left(y_{i}, \frac{1}{2} r_{i}\right)\right)^{q}\left(2 \frac{1}{2} r_{i}\right)^{t} \leq 2^{t} c_{0} \overline{\mathcal{P}}_{\nu, \frac{1}{2} \delta}^{q, t}(E),
$$

and so $\overline{\mathcal{P}}_{\nu, \delta}^{q, t}(\bar{E}) \leq 2^{t} c_{0} \overline{\mathcal{P}}_{\nu, \frac{1}{2} \delta}^{q, t}(E)$. Letting $\delta \searrow 0$ now yields $\overline{\mathcal{P}}_{\nu}^{q, t}(\bar{E}) \leq$ $2^{s} c_{0} \overline{\mathcal{P}}_{\nu}^{q, t}(E)$.

ii) Follows from i).

Lemma 6.5.2. Let $\nu \in \mathcal{P}\left(\mathbb{R}^{d}\right), q \in \mathbb{R}$ and $E \subseteq \mathbb{R}^{d}$. Then

$$
\operatorname{Dim}_{\nu}^{q}(E)=\inf _{E \subseteq \cup \cup_{i=1}^{\infty} E_{i}} \sup _{i} \Delta_{\nu}^{q}\left(E_{i}\right) .
$$


Proof. " $\leq$ " Let $E \subseteq \cup_{i=1}^{\infty} E_{i}$. The monotonicity and countable stability of $\operatorname{Dim}_{\nu}^{q}$ (c.f. [Ol1]) and Proposition 2.1.2 imply that $\operatorname{Dim}_{\nu}^{q}(E) \leq \sup _{i} \operatorname{Dim}_{\nu}^{q}\left(E_{i}\right)$ $\leq \sup _{i} \Delta_{\nu}^{q}\left(E_{i}\right)$ for all coverings $\left(E_{i}\right)_{i}$ of $E$, whence $\operatorname{Dim}_{\nu}^{q}(E) \leq$ $\inf _{E \subseteq \cup_{i=1}^{\infty} E_{i}} \sup _{i} \Delta_{\nu}^{q}\left(E_{i}\right)$.

" $\geq$ " Let $s>\operatorname{Dim}_{\nu}^{q}(E)$. Then $0=\mathcal{P}_{\nu}^{q, s}(E)=\sup _{E \subseteq \cup_{i=1}^{\infty} E_{i}} \sum_{i} \overline{\mathcal{P}}_{\nu}^{q, s}\left(E_{i}\right)$, so that $E \subseteq \cup_{i=1}^{\infty} F_{i}$ for a countable family of sets $F_{i}$ with $\mathcal{\mathcal { P }}_{\nu}^{i \bar{q}, s}\left(F_{i}\right)<\infty$. Hence $\Delta_{\nu}^{q}\left(F_{i}\right) \leq s$ for all $i$, whence $\inf _{E \subseteq \cup_{i=1}^{\infty} E_{i}} \sup _{i} \Delta_{\nu}^{q}\left(E_{i}\right) \leq \sup _{i} \Delta_{\nu}^{q}\left(F_{i}\right) \leq s$ for all $s>\operatorname{Dim}_{\nu}^{q}(E)$.

Lemma 6.5.3. Let $\nu \in \mathcal{P}_{F}\left(\mathbb{R}^{d}\right), q \in \mathbb{R}$ and $E \subseteq \mathbb{R}^{d}$ be compact. If

$$
\Delta_{\nu}^{q}(E \cap U)=\Delta_{\nu}^{q}(E) \text { for all open sets } U \text { with } E \cap U \neq \varnothing,
$$

then

$$
\operatorname{Dim}_{\nu}^{q}(E)=\Delta_{\nu}^{q}(E)
$$

Proof. " $\leq$ " Follows from Proposition 2.1.2.

" $\geq$ " Let $E \subseteq \cup_{i=1}^{\infty} E_{i}$. Since $E \subseteq \cup_{i=1}^{\infty} \overline{E_{i}}$, Baire's category theorem implies that there exists a $j \in \mathbb{N}$ and an open set $V$ such that $\varnothing \neq E \cap V \subseteq \overline{E_{j}}$. Hence $\sup _{i} \Delta_{\nu}^{q}\left(\overline{E_{i}}\right) \geq \Delta_{\nu}^{q}\left(\overline{E_{j}}\right) \geq \Delta_{\nu}^{q}(E \cap V)=\Delta_{\nu}^{q}(E)$. Since the covering $\left(E_{i}\right)_{i}$ of $E$ was arbitrary, the two previous lemmas now imply that

$$
\operatorname{Dim}_{\nu}^{q}(E)=\inf _{E \subseteq \cup_{i} E_{i}} \sup _{i} \Delta_{\nu}^{q}\left(E_{i}\right)=\inf _{E \subseteq \cup_{i} E_{i}} \sup _{i} \Delta_{\nu}^{q}\left(\overline{E_{i}}\right) \geq \Delta_{\nu}^{q}(E) .
$$

We will now use Lemma 6.5.3 to prove that $B_{\mu}=\Lambda_{\mu}$ and thereby establish the first equality in Theorem 4.1.3.ii).

Lemma 6.5.4. Let $\nu, \lambda \in \mathcal{P}\left(\mathbb{R}^{d}\right)$ and let $T: \mathbb{R}^{d} \rightarrow \mathbb{R}^{d}$ be a bi-Lipschitz map, i.e. there exist numbers $c, C \in] 0, \infty[$ such that $c|x-y| \leq|T x-T y| \leq C|x-y|$ for all $x, y \in \mathbb{R}^{d}$. Assume that $T(\operatorname{supp} \nu) \subseteq \operatorname{supp} \lambda$. For $q \in \mathbb{R}$ write

$$
\begin{aligned}
& \underline{J}_{\nu, \lambda}^{q}(T)=\liminf _{r \searrow 0} \inf _{x \in \operatorname{supp} \nu}\left(\frac{\lambda B(T x, c r)}{\nu B(x, r)}\right)^{q}, \\
& \bar{J}_{\nu, \lambda}^{q}(T)=\limsup _{r \searrow 0} \sup _{x \in \operatorname{supp} \nu}\left(\frac{\lambda B(T x, C r)}{\nu B(x, r)}\right)^{q} .
\end{aligned}
$$

Let $q, t \in \mathbb{R}$ and $E \subseteq \operatorname{supp} \nu$. Then
i) $\quad \underline{J}_{\nu, \lambda}^{q}(T) c^{t} \overline{\mathcal{P}}_{\nu}^{q, t}(E) \leq \overline{\mathcal{P}}_{\lambda}^{q, t}(T E) \leq \bar{J}_{\nu, \lambda}^{q}(T) C^{t} \overline{\mathcal{P}}_{\nu}^{q, t}(E)$.
ii) $\quad \underline{J}_{\nu, \lambda}^{q}(T) c^{t} \mathcal{P}_{\nu}^{q, t}(E) \leq \mathcal{P}_{\lambda}^{q, t}(T E) \leq \bar{J}_{\nu, \lambda}^{q}(T) C^{t} \mathcal{P}_{\nu}^{q, t}(E)$.
iii) $\quad \underline{J}_{\nu, \lambda}^{q}(T) c^{t} \mathcal{H}_{\nu}^{q, t}(E) \leq \mathcal{H}_{\lambda}^{q, t}(T E) \leq \bar{J}_{\nu, \lambda}^{q}(T) C^{t} \mathcal{H}_{\nu}^{q, t}(E)$. 
iv) If $0<\underline{J}_{\nu, \lambda}^{q}(T) \leq \bar{J}_{\nu, \lambda}^{q}(T)<\infty$ then $\Delta_{\nu}^{q}(E)=\Delta_{\lambda}^{q}(T E), \operatorname{Dim}_{\nu}^{q}(E)=$ $\operatorname{Dim}_{\lambda}^{q}(T E)$ and $\operatorname{dim}_{\nu}^{q}(E)=\operatorname{dim}_{\lambda}^{q}(T E)$.

Proof. Follows easily from the definitions. See also [O11, Lemma 4.3] where the assertions are proved in the special case where $c=C$.

Lemma 6.5.5. Assume that $S_{\mathbf{i}}\left([0,1]^{d}\right) \cap S_{\mathbf{j}}\left([0,1]^{d}\right)=\varnothing$ for $\mathbf{i} \neq \mathbf{j}$ (this is in particular satisfied if condition (II) holds). Let $\mathbf{i} \in I, x \in K$ and $0<r<\frac{n_{1}}{2 n_{d}}$. Then

$$
\mu\left(S_{\mathbf{i}}(U(x, r))\right)=p_{\mathbf{i}} \mu(U(x, r))
$$

where $U(x, r)$ denotes the open ball with center $x$ and radius $r$.

Proof. The proof of Lemma 6.5.5 is identical to the proof of [O11, Lemma 5.6] and is therefore omitted.

Lemma 6.5.6. Assume that condition (II) holds. Let $q \in \mathbb{R}, n \in \mathbb{N}$ and $\mathbf{i}_{1}, \ldots, \mathbf{i}_{n} \in I$. Then

i) $\underline{J}_{\mu, \mu}^{q}\left(S_{\mathbf{i}_{1}} \circ \cdots \circ S_{\mathbf{i}_{n}}\right)>0$.

ii) $\bar{J}_{\mu, \mu}^{q}\left(S_{\mathbf{i}_{1}} \circ \cdots \circ S_{\mathbf{i}_{n}}\right)<\infty$.

(Here $\underline{J}_{\mu, \mu}^{q}\left(S_{\mathbf{i}_{1}} \circ \cdots \circ S_{\mathbf{i}_{n}}\right)$ and $\bar{J}_{\mu, \mu}^{q}\left(S_{\mathbf{i}_{1}} \circ \cdots \circ S_{\mathbf{i}_{n}}\right)$ are defined in Lemma 6.5.4.)

Proof. It follows from Proposition 6.2.1 and Lemma 6.2.3 that there exists a constant $c>0$ such that

$$
\begin{aligned}
c^{-1} \leq \frac{\mu B\left(x, 2 \frac{n_{d}^{n}}{n_{l}^{n}} r\right)}{\mu B(x, r)} \leq c, \quad c^{-1} & \leq \frac{\mu B\left(x, \frac{1}{2} \frac{n_{1}^{n}}{n_{l}^{n}} r\right)}{\mu B(x, r)} \leq c \\
& \text { for } l=1, d, r>0 \text { and } x \in \operatorname{supp} \mu .
\end{aligned}
$$

As before, $U(x, r)$ denotes the open ball with center $x$ and radius $r$. Write $p=\min _{\mathbf{i}} p_{\mathbf{i}}$ and $\bar{p}=\max _{\mathbf{i}} p_{\mathbf{i}}$. We clearly have $S_{\mathbf{i}}\left(U\left(x, n_{1} r\right)\right) \subseteq U\left(S_{\mathbf{i}}(x), r\right) \subseteq$ $\bar{S}_{\mathbf{i}}\left(U\left(x, n_{d} r\right)\right)$ for $\mathbf{i} \in I, r>0$ and $x \in \operatorname{supp} \mu$, and (6.21) and the previous lemma therefore imply that

$$
\begin{aligned}
\underline{p}^{n} c^{-1} & \leq \underline{p}^{n} \frac{\mu B\left(x, \frac{1}{2} \frac{n_{1}^{n}}{n_{l}^{n}} r\right)}{\mu B(x, r)} \leq \frac{\mu B\left(S_{\mathbf{i}_{1}} \circ \cdots \circ S_{\mathbf{i}_{n}}(x), \frac{1}{n_{l}^{n}} r\right)}{\mu B(x, r)} \\
& \leq \bar{p}^{n} \frac{\mu B\left(x, 2 \frac{n_{d}^{n}}{n_{l}^{n}} r\right)}{\mu B(x, r)} \leq \bar{p}^{n} c
\end{aligned}
$$

for $l=1, d, 0<r<\frac{n_{1}^{n+1}}{2 n_{d}^{n+1}}$ and $x \in \operatorname{supp} \mu$. This proves the lemma.

Proof of the equality $B_{\mu}=\Lambda_{\mu}$ in Theorem 4.1.3.ii). Let $q \in \mathbb{R}$. We must now prove that $B_{\mu}(q)=\Lambda_{\mu}(q)$. Since $B_{\mu}(q)=\operatorname{Dim}_{\mu}^{q}(K)$ and $\Lambda_{\mu}(q)=$ 
$\Delta_{\mu}^{q}(K)$, it suffices, by Lemma 6.5.3, to prove that $\Delta_{\mu}^{q}(K \cap U)=\Delta_{\mu}^{q}(K)$ for all open sets $U$ with $K \cap U \neq \varnothing$. Now let $U$ be an open set with $x=\pi(\omega) \in K \cap U$ where $\omega=\left(\mathbf{i}_{1}, \mathbf{i}_{2}, \ldots\right) \in \Sigma$. Since $S_{\mathbf{i}_{1}} \circ \cdots \circ S_{\mathbf{i}_{m}}(K) \searrow\{x\}$ there exists an integer $n$ such that $S_{\mathbf{i}_{1}} \circ \cdots \circ S_{\mathbf{i}_{n}}(K) \subseteq K \cap U$. Finally, Lemma 6.5.4 and Lemma 6.5.6 show that $\Delta_{\mu}^{q}\left(S_{\mathbf{i}_{1}} \circ \cdots \circ S_{\mathbf{i}_{n}}(K)\right)=\Delta_{\mu}^{q}(K)$, whence $\Delta_{\mu}^{q}(K \cap U) \geq \Delta_{\mu}^{q}\left(S_{\mathbf{i}_{1}} \circ \cdots \circ S_{\mathbf{i}_{n}}(K)\right)=\Delta_{\mu}^{q}(K) \geq \Delta_{\mu}^{q}(K \cap U)$ and so $\Delta_{\mu}^{q}(K \cap U)=\Delta_{\mu}^{q}(K)$.

\subsection{Multifractal box dimensions and generalized Rényi dimen- sions for multifractal Sierpinski sponges.}

In this section we compute the multifractal box dimensions and the generalized Rényi dimensions of $\mu$ assuming condition (II). For $r>0$ we denote the family of approximate cubes with approximate diameter $r$ by $\mathcal{Q}(r)$, i.e.

$$
\mathcal{Q}(r):=\{Q(\omega, r) \mid \omega \in \Sigma\} .
$$

For $\mathbf{i}=\left(i_{1}, \ldots, i_{n}\right) \in \prod_{l=1}^{d}\left\{0, \ldots, n_{l}-1\right\}$ write

$$
\begin{array}{r}
\mathcal{Q}_{\mathbf{i}}(r):=\left\{Q(\omega, r)=\prod_{l=1}^{d}\left[\frac{i_{1, l}}{n_{l}}+\cdots+\frac{i_{k_{l}(r), l}}{n_{l}(r)}, \frac{i_{1, l}}{n_{l}}+\cdots+\frac{i_{k_{l}(r), l}}{n_{l}^{k_{l}(r)}}+\frac{1}{n_{l}^{k_{l}(r)}}\right] \mid\right. \\
\omega=\left(\mathbf{i}_{1}, \mathbf{i}_{2}, \ldots\right) \in \Sigma, \\
\mathbf{i}_{j}=\left(i_{j, 1}, \ldots, i_{j, d}\right) \text { for all } j, \\
\left.i_{k_{1}(r), 1}=i_{1}, \ldots, i_{k_{d}(r), d}=i_{d}\right\} .
\end{array}
$$

Observe that $\left(\mathcal{Q}_{\mathbf{i}}(r)\right)_{\mathbf{i}}$ is a partition of $\mathcal{Q}(r)$, and that

$$
Q^{\prime} \cap Q^{\prime \prime}=\varnothing \text { for all } Q^{\prime}, Q^{\prime \prime} \in \mathcal{Q}_{\mathbf{i}}(r) \text { with } Q^{\prime} \neq Q^{\prime \prime} .
$$

Lemma 6.6.1. Let $q \in \mathbb{R}$. Then

$$
\frac{\log \left(\sum_{Q \in \mathcal{Q}(r)} \mu(Q)^{q}\right)}{-\log r} \rightarrow \gamma(q) \quad \text { as } r \searrow 0
$$

(recall that $\gamma(q)$ is defined in Section 3.1).

Proof. It follows from (6.2) that

$$
\log \left(\sum_{Q \in \mathcal{Q}(r)} \mu(Q)^{q}\right)
$$




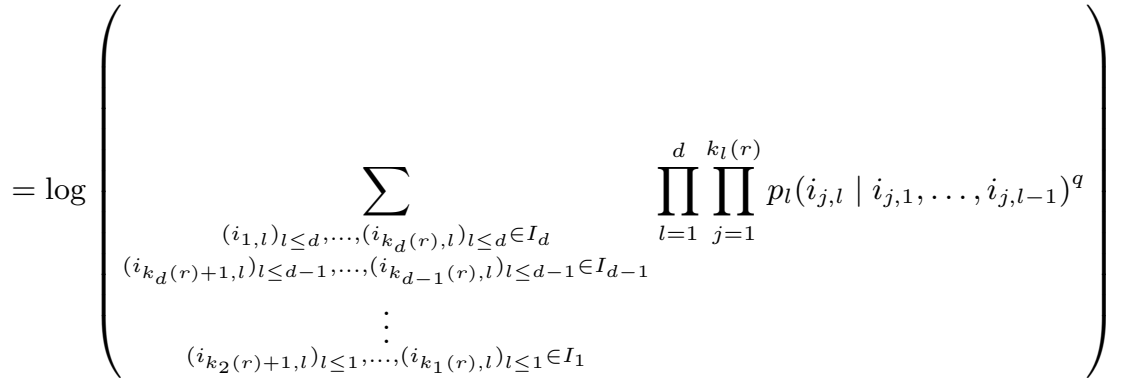

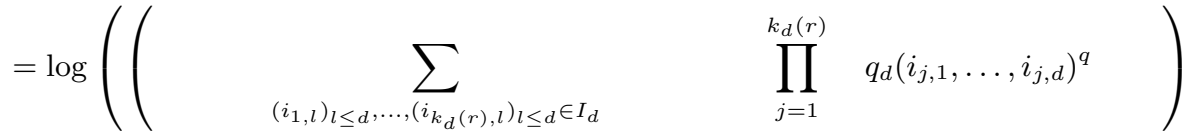

$$
\begin{aligned}
& \left(\sum_{\left(i_{k_{d}(r)+1, l}\right)_{l \leq d-1}, \ldots,\left(i_{k_{d-1}(r), l}\right)_{l \leq d-1} \in I_{d-1}} \prod_{j=k_{d}(r)+1}^{k_{d-1}(r)} q_{d-1}\left(i_{j, 1}, \ldots, i_{j, d-1}\right)^{q}\right) \\
& \left.\left(\sum_{\left(i_{k_{2}(r)+1, l}\right)_{l \leq 1}, \ldots,\left(i_{k_{1}(r), l}\right)_{l \leq 1} \in I_{1}} \prod_{j=k_{2}(r)+1}^{k_{1}(r)} q_{1}\left(i_{j, 1}\right)^{q}\right)\right) .
\end{aligned}
$$

For convenience write $k_{d+1}(r)=0$. Since

$$
\begin{gathered}
\sum_{\left.\left(i_{k_{l+1}(r)+1, m}\right)_{m \leq l}, \ldots, i_{k_{l}(r), m}\right)_{m \leq l} \in I_{l}} \prod_{\substack{j=k_{l+1}(r)+1 \\
k_{l}(r)-k_{l+1}(r)}}^{k_{l}(r)} q_{l}\left(i_{j, 1}, \ldots, i_{j, l}\right)^{q} \\
=\left(\sum_{\left(i_{1}, \ldots, i_{l}\right) \in I_{l}} q_{l}\left(i_{1}, \ldots, i_{l}\right)^{q}\right)^{,}
\end{gathered}
$$

we deduce from Equation (6.23) that

$$
\begin{aligned}
\log \left(\sum_{Q \in \mathcal{Q}(r)} \mu(Q)^{q}\right) & =\log \left(\prod_{l=1}^{d}\left(\sum_{\left(i_{1}, \ldots, i_{l}\right) \in I_{l}} q_{l}\left(i_{1}, \ldots, i_{l}\right)^{q}\right)^{k_{l}(r)-k_{l+1}(r)}\right) \\
& =\sum_{l=1}^{d}\left(k_{l}(r)-k_{l+1}(r)\right) \log \left(\sum_{\left(i_{1}, \ldots, i_{l}\right) \in I_{l}} q_{l}\left(i_{1}, \ldots, i_{l}\right)^{q}\right) .
\end{aligned}
$$

The desired result now follows from (6.24) since $\frac{k_{l}(r)}{-\log r} \rightarrow \frac{1}{\log n_{l}}$ as $r \searrow$ 0 .

Theorem 6.6.2. Let $q \in \mathbb{R}$ and assume that condition (II) holds. Then

$$
\underline{\mathcal{C}}_{\mu}(q)=\overline{\mathcal{C}}_{\mu}(q)=\mathcal{C}_{\mu}(q)=\gamma(q)
$$


Proof. It follows from Proposition 6.2.1 and Lemma 6.3.2 that there exists a constant $c>0$ such that

$$
\begin{gathered}
\left(\frac{\mu Q(\omega, r)}{\mu B\left(\pi(\omega), \frac{1}{n_{1}^{k_{1}(r)+1}}\right)}\right)^{q} \leq c, \quad\left(\frac{\mu B(\pi(\omega), r)}{\mu Q\left(\omega, \frac{1}{n_{1}^{m+1}}\right)}\right)^{q} \leq c \\
\text { for } \omega \in \Sigma, m \in \mathbb{N} \text { and } \frac{1}{n_{1}^{m+1}} \leq \frac{r}{n_{1}+\cdots+n_{d}}<\frac{1}{n_{1}^{m}} .
\end{gathered}
$$

Also observe that for $\mathbf{i} \in \prod_{l=1}^{d}\left\{0, \ldots, n_{l}-1\right\}$,

$$
\forall Q^{\prime}, Q^{\prime \prime} \in \mathcal{Q}_{\mathbf{i}}(r): \operatorname{dist}\left(Q^{\prime}, Q^{\prime \prime}\right) \geq \min _{l} \frac{1}{n_{l}^{k_{l}(r)}}>\frac{1}{n_{1}^{k_{1}(r)+1}}
$$

For $\mathbf{i} \in \prod_{l=1}^{d}\left\{0, \ldots, n_{l}-1\right\}$ let $\mathcal{Q}_{\mathbf{i}}(r)=\left\{Q\left(\omega_{\mathbf{i}, r, j}, r\right) \mid j \in \mathbb{N}\right\}$. Now fix $r>0$. It follows from (6.26) that $\left(B\left(\pi\left(\omega_{\mathbf{i}, r, j}\right), \frac{1}{n_{1}^{k_{1}(r)+1}}\right)\right)_{j}$ is a centered $r$-packing of supp $\mu$, whence (by (6.25))

$$
\begin{aligned}
& \sum_{Q \in \mathcal{Q}(r)} \mu(Q)^{q} \\
& \leq c \sum_{\mathbf{i} \in \prod_{l=1}^{d}\left\{0, \ldots, n_{l}-1\right\}} \sum_{j} \mu\left(B\left(\pi\left(\omega_{\mathbf{i}, k, j}\right), \frac{1}{n_{1}^{k_{1}(r)+1}}\right)\right)^{q} \\
& \leq c \sum_{\mathbf{i} \in \prod_{l=1}^{d}\left\{0, \ldots, n_{l}-1\right\}} S_{\mu, r}^{q}(\operatorname{supp} \mu)=c n_{1} \cdots n_{d} S_{\mu, r}^{q}(\operatorname{supp} \mu) \quad \text { for } r>0 .
\end{aligned}
$$

Next, let $\left(B\left(x_{i}, r\right)\right)_{i}$ be a centered $r$-packing of $\operatorname{supp} \mu$. Choose $\omega_{i} \in \Sigma$ such that $x_{i}=\pi\left(\omega_{i}\right)$. Also choose $m \in \mathbb{N}$ such that $\frac{1}{n_{1}^{m+1}} \leq \frac{r}{n_{1}+\cdots+n_{d}}<\frac{1}{n_{1}^{m}}$. It follows from Proposition 6.2.1 that

$$
Q\left(\omega_{i}, \frac{1}{n_{i}^{m+1}}\right) \subseteq B\left(\pi\left(\omega_{i}\right),\left(n_{1}+\cdots+n_{d}\right) \frac{1}{n_{1}^{m+1}}\right) \subseteq B\left(\pi\left(\omega_{i}\right), r\right)
$$

and so $Q\left(\omega_{i}, \frac{1}{n_{1}^{m}}\right) \neq Q\left(\omega_{j}, \frac{1}{n_{1}^{m}}\right)$ for $i \neq j$. Hence

$$
\sum_{i} \mu\left(B\left(x_{i}, r\right)\right)^{q} \leq c \sum_{i} \mu Q\left(\omega_{i}, \frac{1}{n_{1}^{m+1}}\right)^{q} \leq c \sum_{Q \in \mathcal{Q}\left(n_{1}^{-m}\right)} \mu(Q)^{q}
$$


and so,

$$
S_{\mu, r}^{q}(\operatorname{supp} \mu) \leq c \sum_{Q \in \mathcal{Q}\left(n_{1}^{-m}\right)} \mu(Q)^{q} \quad \text { for } \frac{1}{n_{1}^{m+1}} \leq \frac{r}{n_{1}+\cdots+n_{d}}<\frac{1}{n_{1}^{m}} .
$$

The result now follows from (6.27), (6.28) and Lemma 6.6.1.

Theorem 6.6.3. Let $q \in \mathbb{R}$ and assume that condition (II) holds. Then

$$
\underline{\mathcal{D}}_{\mu}(q)=\overline{\mathcal{D}}_{\mu}(q)=\mathcal{D}_{\mu}(q)=\gamma(q) \text {. }
$$

Proof. Proposition 6.2.1 and Lemma 6.3.2 imply that there exists a number $c>1$ such that

$$
c^{-1} \leq\left(\frac{\mu B(x, r)}{\mu B(y, 2 r)}\right)^{q} \leq c, \quad c^{-1} \leq\left(\frac{\mu B(x, r)}{\mu(Q)}\right)^{q} \leq c
$$

$$
\text { for } r>0, Q \in \mathcal{Q}\left(\frac{1}{n_{1}^{k_{1}(r)}}\right), x \in Q \cap K \text { and } y \in B(x, r) \cap K .
$$

We now divide the proof into two cases.

Case 1: $\gamma(q+1)=\mathcal{D}_{\mu}(q+1)$ for $q \neq 0$. Let $r>0$ and $\left(B\left(x_{i}, r\right)\right)_{i}$ be a centered packing of $K$. Then

$$
\begin{aligned}
\sum_{i} \mu\left(B\left(x_{i}, r\right)\right)^{q+1} & =\sum_{i} \int_{B\left(x_{i}, r\right)}\left(\frac{\mu B\left(x_{i}, r\right)}{\mu B(x, 2 r)}\right)^{q} \mu(B(x, 2 r))^{q} d \mu(x) \\
& \leq c \int_{\operatorname{supp} \mu} \mu(B(x, 2 r))^{q} d \mu(x)
\end{aligned}
$$

whence

$$
S_{\mu, r}^{q+1}(\operatorname{supp} \mu) \leq c \int_{\operatorname{supp} \mu} \mu(B(x, 2 r))^{q} d \mu(x) \quad \text { for } r>0 .
$$

Inequality (6.27) shows that there exists a constant $c_{0}>0$ with $\sum_{Q \in \mathcal{Q}(r)} \mu(Q)^{q+1} \leq c_{0} S_{\mu, r}^{q+1}(\operatorname{supp} \mu)$ for $r>0$, and (6.29) therefore implies that

$$
\begin{aligned}
& \int_{\operatorname{supp} \mu} \mu(B(x, r))^{q} d \mu(x) \leq \sum_{Q \in \mathcal{Q}\left(\frac{1}{n_{1}^{k_{1}(r)}}\right)} \int_{Q} \mu(B(x, r))^{q} d \mu(x) \\
& \leq c \sum_{Q \in \mathcal{Q}\left(\frac{1}{n_{1}^{k_{1}(r)}}\right)} \mu(Q)^{q+1} \leq c_{0} c S_{\mu, n_{1}^{-k_{1}(r)}}^{q+1}(\operatorname{supp} \mu) .
\end{aligned}
$$


The result now follows from (6.30), (6.31) and Theorem 6.6.2.

Case 2: $\gamma(1)=\mathcal{D}_{\mu}(1)$. Since $\gamma(1)=0, \underline{\mathcal{D}}_{\mu}(q)=(1-q) \underline{D}_{\mu}^{q-1}$ and $\overline{\mathcal{D}}_{\mu}(q)=$ $(1-q) \bar{D}_{\mu}^{q-1}$ with $0 \leq \underline{D}_{\mu}^{0} \leq \bar{D}_{\mu}^{0}$, it suffices to prove that $\bar{D}_{\mu}^{0}<\infty$. We have, using (6.29),

$$
\begin{aligned}
\int_{\operatorname{supp} \mu} \log \mu(B(x, r)) d \mu(x) \geq & \sum_{Q \in \mathcal{Q}\left(n_{1}^{-k_{1}(r)}\right)} \int_{Q} \log \mu(B(x, r)) d \mu(x) \\
\geq & \sum_{Q \in \mathcal{Q}\left(n_{1}^{-k_{1}(r)}\right)} \int_{Q} \log \left(c^{-1} \mu(Q)\right) d \mu(x) \\
= & -\log c \sum_{\mathbf{i} \in \prod_{l=1}^{d}\left\{0, \ldots, n_{l}-1\right\}} \sum_{Q \in \mathcal{Q}_{\mathbf{i}}\left(n_{1}^{-k_{1}(r)}\right)} \mu(Q) \\
& +\sum_{Q \in \mathcal{Q}\left(n_{1}^{-k_{1}(r)}\right)} \mu(Q) \log \mu(Q) \\
\geq & -n_{1} \cdots n_{d} \log c-\log \operatorname{card} \mathcal{Q}\left(n_{1}^{-k_{1}(r)}\right)
\end{aligned}
$$

since $\sum_{Q \in \mathcal{Q}_{\mathbf{i}}\left(n_{1}^{-k_{1}(r)}\right)} \mu(Q)=\mu\left(\cup_{Q \in \mathcal{Q}_{\mathbf{i}}\left(n_{1}^{-k_{1}(r)}\right)} Q\right) \leq 1$, whence

$$
\begin{aligned}
\bar{D}_{\mu}^{0} & \leq \limsup _{r \searrow 0}\left(-n_{1} \cdots n_{d} \frac{\log c}{\log \left(n_{1}^{-k_{1}(r)}\right)}-\frac{\log \operatorname{card} \mathcal{Q}\left(n_{1}^{-k_{1}(r)}\right)}{\log \left(n_{1}^{-k_{1}(r)}\right)}\right) \\
& \leq \bar{C}(K) \leq d<\infty
\end{aligned}
$$

Proof of Corollary 4.1.2.ii). An inspection of the proof of Theorem 6.6.2 shows that the only place where condition (II) was used, was in order to establish Equations (6.25). However, for $q=0$ Equations (6.25) are obviously satisfied. Hence Theorem 6.6.2 holds for $q=0$ without assuming condition (II), i.e. we have $\gamma(0)=\mathcal{C}_{\mu}(0)=C(\operatorname{supp} \mu)=C(K)$ without assuming condition (II). This proves Corollary 4.1.2.i).

Proof of the equality $\Lambda_{\mu}=\gamma$ in Theorem 4.1.3.ii). $\quad$ It follows from Theorem 6.6.2 that $\gamma=\mathcal{C}_{\mu}=\overline{\mathcal{C}}_{\mu}$, and it follows from Theorem 2.2.1 and Lemma 6.2.3 that $\overline{\mathcal{C}}_{\mu}=\Lambda_{\mu}$.

Proof of Theorem 4.1.3.iii). Follows from Theorem 6.6.2 and Theorem 6.6.3. 
6.7. A sufficient condition for the multifractal measures $\mathcal{H}_{\mu}^{q, \beta(q)}$ and $\mathcal{P}_{\mu}^{q, \beta(q)}$ to be positive and finite.

This section investigates the multifractal Hausdorff and packing measure at the critical dimensions.

Proposition 6.7.1. Let $q \in \mathbb{R}$ and assume that condition $\left(\mathrm{I}_{q}\right)$ holds. Then

i) $\underline{D}(q):=\inf _{\omega \in \Sigma} \inf _{r>0} D_{r}(q ; \omega)^{k_{1}(r)}>0$.

ii) $\bar{D}(q):=\sup _{\omega \in \Sigma} \sup _{r>0} D_{r}(q ; \omega)^{k_{1}(r)}<\infty$.

Proof. Let $r>0$ and $\omega=\left(\mathbf{i}_{1}, \mathbf{i}_{2}, \ldots\right) \in \Sigma$. It follows from condition $\left(\mathrm{I}_{q}\right)$ that there exist strictly positive numbers $u_{1}, \ldots, u_{d}$ such that $u_{\mathbf{i}}(q ; l)=$ $n_{l}^{\beta_{i_{1} \ldots i_{l}}(q)}=u_{l}$ for all $l \in\left\{k \mid \alpha_{k} \neq 1\right\}$ and $\left(i_{1}, \ldots, i_{l}\right) \in I_{l}$. We thus deduce that $a_{n}(q ; \omega, l)=u_{\omega \mid n}(q ; l)^{\alpha_{1} \cdots \alpha_{l-1} \frac{1}{n}}=u_{l}^{\alpha_{1} \cdots \alpha_{l-1}}$ for all $l \in\left\{k \mid \alpha_{k} \neq 1\right\}$ and $n \in \mathbb{N}$. It now follows from Proposition 6.1.3 that

$$
D_{r}(q ; \omega)^{k_{1}(r)}=\prod_{\substack{l \\ \alpha_{l} \neq 1}} u_{l}^{k_{l}(r)-\frac{1}{\alpha_{l}} k_{l+1}(r)} .
$$

Finally, since $-1 \leq k_{l}(r)-\frac{1}{\alpha_{l}} k_{l+1}(r) \leq \frac{1}{\alpha_{l}},(6.32)$ implies that

$$
\prod_{\substack{l \\ \alpha_{l} \neq 1}} \min \left(u_{l}^{-1}, u_{l}^{\frac{1}{\alpha_{l}}}\right) \leq D_{r}(q ; \omega)^{k_{1}(r)} \leq \prod_{\substack{l \\ \alpha_{l} \neq 1}} \max \left(u_{l}^{-1}, u_{l}^{\frac{1}{\alpha_{l}}}\right) .
$$

Theorem 6.7.2. Let $q \in \mathbb{R}$ and assume that conditions $\left(\mathrm{I}_{q}\right)$ and (II) hold. Then

i) There exist constants $0<\underline{c} \leq \bar{c}<\infty$ such that

$$
\underline{c} \mu^{q} \leq \mathcal{H}_{\mu}^{q, \beta(q)}\left\llcorner\operatorname{supp} \mu \leq \mathcal{P}_{\mu}^{q, \beta(q)}\left\llcorner\operatorname{supp} \mu \leq \bar{c} \mu^{q} .\right.\right.
$$

ii) $\overline{\mathcal{P}}_{\mu}^{q, \beta(q)}(\operatorname{supp} \mu)<\infty$.

Proof. Let $\underline{D}(q)$ and $\bar{D}(q)$ be defined as in Proposition 6.7.1, and note that Proposition 6.7.1 implies that $0<\underline{D}(q) \leq \bar{D}(q)<\infty$.

Proof of: There exists a constant $\underline{c}>0$ such that

$$
\underline{c} \mu^{q} \leq \mathcal{H}_{\mu}^{q, \beta(q)}\llcorner\operatorname{supp} \mu .
$$

It follows Proposition 6.2.1 and Lemma 6.2.3 that there exists a constant $c_{1}>0$ such that 


$$
\begin{aligned}
& \left(\frac{\mu B(\pi(\omega), r)}{\mu Q\left(\omega, \frac{1}{n_{1}^{m}}\right)}\right)^{q} \geq c_{1}, \quad(2 r)^{\beta(q)} \geq c_{1}\left(\frac{1}{n_{1}^{m}}\right)^{\beta(q)} \\
& \qquad \quad \text { for } \omega \in \Sigma, m \in \mathbb{N} \text { and } \frac{1}{2 n_{1}^{m+1}} \leq r<\frac{1}{2 n_{1}^{m}}
\end{aligned}
$$

Let $\underline{c}=c_{1}^{2} \bar{D}(q)^{-1}$.

Let $B$ be a Borel subset of $\operatorname{supp} \mu$. Now fix $\delta>0$ and let $\left(B\left(x_{i}, r_{i}\right)\right)_{i \in \mathbb{N}}$ be a centered $\delta$-covering of $B$. For each $i \in \mathbb{N}$ choose $\omega_{i} \in \Sigma$ such that $\pi\left(\omega_{i}\right)=x_{i}$, and let $m_{i}$ be the unique integer satisfying

$$
\frac{1}{2 n_{1}^{m_{i}+1}} \leq r_{i}<\frac{1}{2 n_{1}^{m_{i}}} .
$$

It follows from (6.33) and (6.34) that

$$
\begin{aligned}
\sum_{i} \mu\left(B\left(x_{i}, r_{i}\right)\right)^{q}\left(2 r_{i}\right)^{\beta(q)} & \geq c_{1}^{2} \sum_{i} \frac{1}{D_{n_{1}^{-\left(m_{i}\right)}}\left(q ; \omega_{i}\right)^{k_{1}\left(\frac{1}{n_{1}^{m_{i}}}\right)}} \mu^{q}\left(Q\left(\omega_{i}, \frac{1}{n_{1}^{m_{i}}}\right)\right) \\
& \geq c_{1}^{2} \frac{1}{\bar{D}(q)} \sum_{i} \mu^{q}\left(Q\left(\omega_{i}, \frac{1}{n^{m_{i}}}\right)\right) \\
& \geq \underline{c} \mu^{q}\left(\cup_{i} B\left(x_{i}, r_{i}\right)\right) \geq \underline{c} \mu^{q}(B) .
\end{aligned}
$$

Hence $\mathcal{H}_{\mu}^{q, \beta(q)}(B) \geq \overline{\mathcal{H}}_{\mu}^{q, \beta(q)}(B) \geq \overline{\mathcal{H}}_{\mu, \delta}^{q, \beta(q)}(B) \geq \underline{c} \mu^{q}(B)$.

Proof of: $\mathcal{H}_{\mu}^{q, \beta(q)} \operatorname{Lupp} \mu \leq \mathcal{P}_{\mu}^{q, \beta(q)} \operatorname{Lsupp} \mu$. Follows immediately from Lemma 6.2.3 and Proposition 2.1.2.

Proof of: There exists a constant $\bar{c}<\infty$ such that

$$
\begin{gathered}
\mathcal{P}_{\mu}^{q, \beta(q)}\left\llcorner\operatorname{supp} \mu \leq \bar{c} \mu^{q},\right. \\
\overline{\mathcal{P}}_{\mu}^{q, \beta(q)}(\operatorname{supp} \mu) \leq \bar{c} .
\end{gathered}
$$

It follows Proposition 6.2.1 and Lemma 6.2.3 that there exists a constant $c_{2}>0$ such that

$$
\begin{aligned}
\left(\frac{\mu B(\pi(\omega), r)}{\mu Q\left(\omega, \frac{1}{n_{1}^{m+1}}\right)}\right)^{q} \leq c_{2}, \quad(2 r)^{\beta(q)} \leq c_{2}\left(\frac{1}{n_{1}^{m+1}}\right)^{\beta(q)} & \\
\text { for } \omega & \in \Sigma, m \in \mathbb{N} \text { and } \frac{1}{n_{1}^{m+1}} \leq \frac{r}{n_{1}+\cdots+n_{d}}<\frac{1}{n_{1}^{m}}
\end{aligned}
$$


Let $\bar{c}=c_{2}^{2} \bar{D}(q)^{-1}$.

Let $G$ be an open subset of $\mathbb{R}^{d}$ and let $C$ be a compact subset of $G$. Clearly $\delta_{G, C}:=\frac{1}{2} \operatorname{dist}\left(C, \mathbb{R}^{d} \backslash G\right)>0$. Now let $\left(B\left(x_{i}, r_{i}\right)\right)_{i}$ be a centered $\delta_{G, C}$-packing of $C \cap \operatorname{supp} \mu$. For each $i$ choose $\omega_{i} \in \Sigma$ such that $\pi\left(\omega_{i}\right)=x_{i}$, and let $m_{i}$ be the unique integer satisfying

$$
\frac{1}{n_{1}^{m_{i}+1}} \leq \frac{r_{i}}{n_{1}+\cdots+n_{d}}<\frac{1}{n_{1}^{m_{i}}}
$$

It follows from (6.35), (6.36) and the inclusion $\cup_{i} B\left(x_{i}, r_{i}\right) \subseteq G$ that

$$
\begin{aligned}
& \sum_{i} \mu\left(B\left(x_{i}, r_{i}\right)\right)^{q}\left(2 r_{i}\right)^{\beta(q)} \\
& \leq c_{2}^{2} \sum_{i} \frac{1}{D_{n_{1}^{-\left(m_{i}+1\right)}}\left(q ; \omega_{i}\right)^{k_{1}\left(\frac{1}{n_{1}^{m_{i}+1}}\right)} \mu^{q}\left(Q\left(\omega_{i}, \frac{1}{n_{1}^{m_{i}+1}}\right)\right)} \\
& \leq c_{2}^{2} \frac{1}{\underline{D}(q)} \sum_{i} \mu^{q}\left(Q\left(\omega_{i}, \frac{1}{n^{m_{i}+1}}\right)\right) \\
& \leq \bar{c} \mu^{q}\left(\cup_{i} B\left(x_{i}, r_{i}\right)\right) \leq \bar{c} \mu^{q}(G) .
\end{aligned}
$$

We thus deduce that $\overline{\mathcal{P}}_{\mu}^{q, \beta(q)}(C \cap \operatorname{supp} \mu) \leq \overline{\mathcal{P}}_{\mu, \delta_{G}, C}^{q, \beta(q)}(C \cap \operatorname{supp} \mu) \leq \bar{c} \mu^{q}(G)$. Hence

$$
\left(\mathcal{P}_{\mu}^{q, \beta(q)}\llcorner\operatorname{supp} \mu)(C) \leq \overline{\mathcal{P}}_{\mu}^{q, \beta(q)}(C \cap \operatorname{supp} \mu) \leq \bar{c} \mu^{q}(G)\right.
$$

for all open sets $G$ and all compact sets $C$ with $C \subseteq G$.

By letting $G=\mathbb{R}^{d}$ and $C=\operatorname{supp} \mu$ in $(6.37)$ we obtain $\overline{\mathcal{P}}_{\mu}^{q, \beta(q)}(\operatorname{supp} \mu) \leq$ $\bar{c} \mu^{q}\left(\mathbb{R}^{d}\right)=\bar{c}$.

Since $\mathcal{P}_{\mu}^{q, \beta(q)}(\operatorname{supp} \mu) \leq \overline{\mathcal{P}}_{\mu}^{q, \beta(q)}(\operatorname{supp} \mu) \leq \bar{c}<\infty, \mathcal{P}_{\mu}^{q, \beta(q)}$ Lsupp $\mu$ is a finite Borel measure and thus regular. Equation (6.37) together with regularity of $\mathcal{P}_{\mu}^{q, \beta(q)}\left\llcorner\operatorname{supp} \mu\right.$ and $\mu^{q}$ now imply that $\mathcal{P}_{\mu}^{q, \beta(q)}\left\llcorner\operatorname{supp} \mu \leq \bar{c} \mu^{q}\right.$.

Proof of Theorem 4.1.8. Follows immediately from Theorem 6.7.2, Theorem 6.3.2 and Lemma 6.3.3.

Proof of Theorem 4.1.9. $\quad$ i) The assertion in i) follows from Theorem 6.3.2 and Lemma 6.3 .3 by noticing that

$$
\alpha(1)=\zeta(1)=-\sum_{l=1}^{d} \frac{1}{\log n_{l}} \sum_{\left(i_{1}, \ldots, i_{l}\right) \in I_{l}} q_{l}\left(i_{1}, \ldots, i_{l}\right) \log p_{l}\left(i_{l} \mid i_{1}, \ldots, i_{l-1}\right) .
$$


ii) Follows from Lemma 6.3.3 since condition (II) together with Proposition

6.2.1 show that $\lim _{r \backslash 0} \frac{\log \mu Q(\omega, r)}{\log r}=\alpha$ if and only if $\lim _{r \backslash 0} \frac{\log \mu B(\pi(\omega), r)}{\log r}=\alpha$ for $\omega \in \Sigma$ and $\alpha \geq 0$.

\section{References}

[AP] M. Arbeiter and N. Patzschke, Random self-similar multifractals, preprint, 1994.

[Be] T. Bedford, Crinkly curves, Markov partitions and box dimensions in self-similar sets, Ph.D. dissertation, University of Warwick, 1984.

[CM] R. Cawley and R.D. Mauldin, Multifractal decomposition of Moran fractals, Advances in Mathematics, 92 (1992), 196-236.

[Cu1] C.D. Cutler, Some results on the behavior and estimation of the fractal dimensions of distributions on attractors, Journal of Statistical Physics, 62 (1991), 651-708.

$[\mathrm{Cu} 2] \_$, Measure disintegrations with respect to $\sigma$-stable monotone indices and pointwise representation of packing dimension, Proceedings of the 1990 Measure Theory Conference at Oberwolfach, Supplemento Ai Rendiconti del Circolo Mathematico di Palermo, Ser. II, No. 28 (1992), 319-340.

[EM] G.A. Edgar and R.D. Mauldin, Multifractal decompositions of digraph recursive fractals, Proc. London Math. Soc., 65 (1992), 604-628.

[Fa1] K.J. Falconer, The Hausdorff dimension of self-affine fractals, Math. Proc. Camb. Phil. Soc., 103 (1988), 339-350.

[Fa2] , Fractal geometry-mathematical foundations and applications, John Wiley \& Sons, 1990.

[Fa3] , The dimension of self-affine fractals II, Math. Proc. Camb. Phil. Soc., 111 (1992), 169-179.

[Fa4] , The multifractal spectrum of statistically self-similar measures, Journal of Theoretical Probability, 7 (1994), 681-702.

[GH] J.S. Geronimo and D. Hardin, An exact formula for the measure dimension associated with a class of piecewise linear maps, Constr. Approx., 5 (1989), 89-98.

[GP] P. Grassberger and I. Procaccia, Characterization of strange attractors, Phys. Rev. Lett., 50 (1983), 346-349.

[Ha] H. Haase, A survey of the dimensions of measures, Proceedings of the conference 'Topology and Measure VI', Warnemünde, Germany, August 1991 (eds. C. Bandt, J. Flachsmeyer and H. Haase), in Mathematical Research, 66, 66-75, Topology, Measures, and Fractals, Akademie Verlag, 1992.

[HJKPS] T.C. Halsey, M.H. Jensen, L.P. Kadanoff, I. Procaccia and B.J. Shraiman, Fractal measures and their singularities: The characterization of strange sets, Phys. Rev. A, 33 (1986), 1141-1151.

[HP] H. Hentschel and I. Procaccia, The infinite number of generalized dimensions of fractals and strange attractors, Physica, 8D (1983), 435-444.

$[\mathrm{Hu}]$ J. Hutchinson, Fractals and self-similarity, Indiana Univ. Math. J., 30 (1981), 713-747.

[KP] R. Kenyon and Y. Peres, Measures of full dimension on affine-invariant sets, Ergodic Theory and Dynamical Systems, 16 (1996), 307-323. 
[Ki] J. King, The singularity spectrum for general Sierpinski carpets, Advances in Mathematics, 116 (1995), 1-8.

[Mat] P. Mattila, Geometry of sets and measures in Euclidean spaces, Cambridge University Press, 1995.

[McM] C. McMullen, The Hausdorff dimension of general Sierpinski carpets, Nagoya Math. J., 96 (1984), 1-9.

[Ol1] L. Olsen, A multifractal formalism, Advances in Mathematics, 116 (1995), 82-196.

[Ol2] _ Random geometrically graph directed self-similar multifractals, Pitman Research Notes in Mathematics Series, Vol. 307, Longman Scientific \& Technical, 1994.

[Pe1] Y. Peres, The self-affine carpets of McMullen and Bedford have infinite Hausdorff measure, Math. Proc. Cambridge Phil. Soc., to appear.

[Pe2] The packing measure of self-affine carpets, Math. Proc. Cambridge Phil. Soc., 115 (1994), 437-450.

[Pes1] Ya. Pesin, Generalized spectrum for the dimension: The approach based on Carathéodory's construction, in 'Constantin Carathéodory: An international tribute', World Sci. Publishing, Teaneck, (1991), 1108-1119.

[Pes2] _ On rigorous mathematical definitions of correlation dimension and generalized spectrum for dimensions, Journal of Statistical Physics, 71 (1993), 529-547.

[RT] X.S. Raymond and C. Tricot, Packing regularity of sets in n-space, Math. Proc. Camb. Phil. Soc., 103 (1988), 133-145.

[Ri1] R.H. Riedi, An improved multifrcatal formalism and self-affine measures, Ph.D. dissertation, ETH Zurich, Diss. ETH No. 10077, 1993.

[Ri2]__ Multifractal formalism for infinite multinomial measures, preprint, 1994.

[RV] A. Roberts and D. Varberg, Convex functions, Academic Press, 1973.

[SS] J. Schmeling and R. Siegmund-Schultze, The singularity spectrum of self-affine fractals with a Bernoulli measure, preprint, 1992.

[St] R.S. Strichartz, Self-similar measures and their Fourier transforms III, Indiana Univ. Math. J., 42 (1992), 367-411.

[TT] S.J. Taylor and C. Tricot, Packing measure, and its evaluation for a Brownian path, Trans. Amer. Math. Soc., 288 (1985), 679-699.

[Tr] C. Tricot, Two definitions of fractional dimension, Math. Proc. Camb. Phil. Soc., 91 (1982), 57-74.

[Yo] L.-S. Young, Dimension, entropy and Lyapunov exponents, Ergod. Th. \& Dynam. Sys., 2 (1982), 109-124.

Received September 18, 1995 and revised July 21, 1997. The author was supported by grant 11-9421-2 PD from the Danish Natural Science Research Council.

University of St. AndREWs

St. Andrews, FifE KY16 9SS

SCOTLAND

E-mail address: lo@st-and.ac.uk 